Pacific Journal of Mathematics

COMPATIBLE PEIRCE DECOMPOSITIONS OF JORDAN

Teri 


\title{
COMPATIBLE PEIRCE DECOMPOSITIONS OF JORDAN TRIPLE SYSTEMS
}

\section{Kevin MCCRIMmon}

\begin{abstract}
Jordan triple systems and pairs do not in general possess unit elements, so that certain standard Jordan algebra methods for studying derivations, extensions, and bimodules do not carry over to triples. Unit elements usually arise as a maximal sum of orthogonal idempotents. In Jordan triple systems such orthogonal sums of tripotents are not enough: in order to "cover" the space one must allow families of tripotents which are orthogonal or collinear. We show that well behaved triples and pairs do possess covering systems of mixed tripotents, and that for many purposes such nonorthogonal families serve as an effective substitute for a unit element. In particular, they can be used to reduce the cohomology of a direct sum to the cohomology of the summands.
\end{abstract}

Throughout we consider Jordan triple systems $J$ over an arbitrary ring $\Phi$ of scalars, having product $P(x) y$ quadratic in $x$ and linear in $y$ with polarized trilinear product $\{x y z\}=P(x, z) y=$ $L(x, y) z$. For easy reference we record the following standard identities satisfied by the multiplications in a Jordan triple system:

$$
\begin{gathered}
P(P(x) y)=P(x) P(y) P(x) \\
P(x) L(y, x)=P(P(x) y, x)=L(x, y) P(x) \\
L(P(x) y, y)=L(x, P(y) x) \\
L(x, y) P(z)+P(z) L(y, x)=P(\{x y z\}, z) \\
{[L(x, y), L(z, w)]=L(\{x y z\}, w)-L(z,\{y x w\})} \\
P(x, y) P(z)=L(x, z) L(y, z)-L(x, P(z) y), \\
P(z) P(x, y)=L(z, x) L(z, y)-L(P(z) x, y) \\
P(P(x) y, z)=P(x, z) L(y, x)-L(z, y) P(x) \\
=L(x, y) P(x, z)-P(x) L(z, y) \\
P(\{x y z\})+P(P(x) P(y) z, z)=P(x) P(y) P(z)+P(z) P(y) P(x) \\
+L(x, y) P(z) L(y, x)
\end{gathered}
$$

(see [2], [3], [8] for basic facts about Jordan triple systems).

We recall the 3 basic examples of Jordan triple systems. The rectangular $p \times q$ matrices with entries in a unital algebra $D$ with 
involution $a \rightarrow \bar{a}$ become a triple system

$$
M_{p, q}(D): P(x) y=x\left(\bar{y}^{t} x\right) \quad(\text { if } p \leqq q) \quad \text { or } \quad\left(x \bar{y}^{t}\right) x(\text { if } p \geqq q) .
$$

We always assume $p+q \geqq 3$ since $M_{1,1}(D)$ is just $D$; here $D$ must be alternative (though the involution is arbitrary), and if $p+q \geqq 4$ must even be associative. When $D$ is associative the triple structure is given by $P(x) y=x \bar{y}^{t} x$. If $p=q=n M_{n, n}(D)$ is just the $j$-isotope $P(x) y=U(x) y^{j}$ of the unital Jordan algebra $M_{n}(D)$ of $n \times n$ matrices, with respect to the adjoint involution $y^{j}=\bar{y}^{t}$. In general we have a decomposition $M_{p, q}(D)=\bigoplus_{1 \leqq i \leqq p, 1 \leqq j \leqq q} D E_{i j}$ where

$$
\begin{aligned}
& P\left(a E_{i j}\right) b E_{i j}=a(\bar{b} a) E_{i j}=(a \bar{b}) a E_{i j} \\
& \left\{a E_{i j} b E_{i j} c E_{i l}\right\}=a(\bar{b} c) E_{i l} \\
& \left\{c E_{k j} b E_{i j} a E_{i j}\right\}=(c \bar{b}) a E_{k j} \\
& \left\{a E_{i j} b E_{k j} c E_{k l}\right\}=a(\bar{b} c) E_{i l}=(a \bar{b}) c E_{i l}
\end{aligned}
$$

for $i \neq l, j \neq k$, while all other "unlinked" products $P\left(a E_{i j}\right) b E_{r s}$ $((r, s) \neq(i, j))$ and $\left\{a E_{i j} b E_{r s} c E_{k l}\right\}((r, s) \neq(k, j),(i, l))$ are zero.

The alternating matrices (those skew-symmetric $X^{t}=-X$ with diagonal entries $X_{i i}=0$ ) over a commutative associative algebra $C$ form a Jordan triple system $A_{n}(C)$ under the product ${ }^{1}$

$$
A_{n}(C): P_{A}(x) y=x y^{t} x=-x y x .
$$

When $C$ has an involution $c \rightarrow \bar{c}$, the map $X^{j}=\bar{X}^{t}$ is an involution on $A_{n}(C)$, and we can form the $j$-isotope

$$
S_{n}(C): P_{S}(x) y=P_{A}(x) y^{j}=x \bar{y}^{t} x=-x \bar{y} x,
$$

which is called the symplectic triple system $S_{n}(C)$. We may view $A_{n}(C)$ as the special case of a symplectic system $S_{n}(C)$ where $C$ has trivial involution. Note that the involution is not used in determining the matrices in $S_{n}(C)$, only in defining the product: both

1 We remark that the alternating matrices also form a subsystem of $M_{n}(C)$ under the product $P_{M}(x) y=x y x$, but in general (e.g., over $\boldsymbol{R}$ ) this system contains no tripotents at all, whereas under $P_{A}$ the symplectic matrix units $F_{i j}=E_{i j}-E_{j i}$ always are tripotents. We also remark that the space of all skew-hermitian matrices $\bar{X}^{t}=-X$ forms a Jordan triple system $S k\left(M_{n}(C)\right)$ under $P(x) y=x y^{t} x=-x \bar{y} x$. For even $n=2 m$ this is just an isotope of the Jordan algebra $H_{2 m}(C, \sigma)$ of symmetric elements relative to the symplectic involution $X^{o}=S \bar{X}^{t} S^{-1}=-S \bar{X}^{t} S$ ( $S$ the standard symplectic matrix) under $U(x) y=x y x$, since $X \rightarrow S X$ is an isomorphism of $S k\left(M_{2 m}(C)\right)$ with the isotope $P_{S}(x) y=-$ $U(x) U(S) y=-x(S y S) x$. In particular, in characteristic $\neq 2$ alternating is the same as skew so $A_{2 m}(C)$ and $S_{2 m}(C)$ are isotopes of $H_{2 m}(C, \sigma)$, and only the case where $n$ is odd produces something new. (Note that for a nontrivial involution the space of hermitianalternating matrices spanned by the $c\langle i j\rangle=c E_{i j}-\bar{c} E_{j i}$ for $i \neq j$ does not form a triple system under $X \bar{Y}^{t} X$, since $\{1\langle 12\rangle 1\langle 23\rangle c\langle 31\rangle\}=(\tilde{c}-c) E_{11}$ is alternating only when $\left.\bar{c}-c=0\right)$. 
$A_{n}(C)$ and $S_{n}(C)$ consist of the same alternating matrices. In terms of the basis elements $a F_{i j}=a\left(E_{i j}-E_{j i}\right)=-a F_{j i}(a \in C, i \neq j)$ the product takes the explicit form

$$
\begin{aligned}
& P\left(a F_{i j}\right) b F_{i j}=a \bar{b} a F_{i j} \\
& \left\{a F_{i j} b F_{i j} c F_{i k}\right\}=a \bar{b} c F_{j k} \\
& \left\{a F_{i j} b F_{k j} c F_{k i}\right\}=0 \\
& \left\{a F_{i j} b F_{k j} c F_{k l}\right\}=a \bar{b} c F_{i l}
\end{aligned}
$$

for distinct $i, j, k, l$, while all other "unlinked" products $P\left(a F_{i j}\right) b F_{k l}$ $((k, l) \neq(i, j),(j, i))$ and $\left\{a F_{i j} b F_{k l} c F_{r s}\right\}((k, l),(l, k) \notin\{i, j\} \times\{r, s\})$ are zero. We are interested only in $S_{n}(C)$ for $n \geqq 4$, since for smaller $n$

$$
S_{1}(C)=0, S_{2}(C) \cong C, S_{3}(C) \cong M_{1,3}(C)
$$

because $a F_{12}+b F_{13}+c F_{23} \rightarrow a E_{11}+b E_{12}+c E_{13}$ is an isomorphism of $S_{3}(C)$ on $M_{1,3}(C)$, under which the symplectic units $\left\{F_{12}, F_{13}, F_{23}\right\}$ correspond to the rectangular row units $\left\{E_{11}, E_{12}, E_{13}\right\}$.

Just as general symplectic matrix systems are obtained as isotopes of alternating systems, so we can obtain general hermitian triple systems as isotopes of the Jordan algebra $H_{n}\left(D, D_{0}\right)$ of $n \times n$ hermitian matrices $X^{* t}=X$ over $D$ whose diagonal entries lie in a given ample subspace $D_{0}$ (a subspace of symmetric elements in the nucleus of $D$ containing 1 and closed under $a D_{0} a^{*} \subset D_{0}$ for all $a \in D$ ). Here $D$ is forced to be alternative with $D_{0}$ contained in the nucleus if $n \geqq 3$ and associative if $n \geqq 4$. If $j$ is an automorphism of $D$ of period 2 commuting with the given involution $*$ and leaving $D_{0}$ invariant, we can define the hermitian Jordan triple system to consist of the same hermitian matrices under the $j$-isotopic product $U(x) y^{j}$ where $y^{j}$ denotes the result of applying $j$ to all the entries of the matrix $y$. As in the symplectic case, $j$ is used only in determining the product, not the matrices. By commutativity, $\bar{a}=$ $a^{* j}$ defines another involution on $D$, and for $*$-hermitian matrices $X=X^{* t}$ we have $X^{j}=X^{* j t}=\bar{X}^{t}$, so the product can also be written as $H_{n}\left(D, D_{0}, j\right): P_{j}(x) y=U(x) y^{j}=U(x) \bar{y}^{t}\left(=x \bar{y}^{t} x\right.$ if $D$ is associative). Whether $D$ is associative or not, $H_{n}\left(D, D_{0}, j\right)$ is spanned by the $a[i j]=a E_{i j}+a^{*} E_{j i}, a_{0}[i i]=a_{0} E_{i i}$ for $a \in D, a_{0} \in D_{0}$, with products

$$
\begin{aligned}
& P\left(a_{0}[i i]\right) b_{0}[i i]=a_{0}\left(b_{0}^{j} a_{0}\right)[i i]=a_{0}\left(\bar{b}_{0} a_{0}\right)[i i] \\
& P(a[i j]) b[i j]=a\left(b^{* j} a\right)[i j]=a(\bar{b} a)[i j] \\
& P(a[i j]) b_{0}[j j]=a\left(b_{0}^{j} a^{*}\right)[i i]=a\left(\bar{b}_{0} \bar{a}^{j}\right)[i i] \\
& \{a[i j] b[i j] c[i k]\}=a\left(b^{* j} c\right)[i k]=a(\bar{b} c)[i k] \\
& \{a[i j] b[k j] c[k i]\}=t\left(a\left(b^{* j} c\right)\right)[i i]=t(a(\bar{b} c))[i i] \\
& \quad(k=i \text { allowed })
\end{aligned}
$$




$$
\begin{aligned}
& \{a[i j] b[k j] c[k l]\}=a\left(b^{* j} c\right)[i l]=a(\bar{b} c)[i l] \\
& \quad(k=j \text { or } i=j \text { or } k=i=j \text { or } i=j, k=l \text { allowed })
\end{aligned}
$$

for distinct indices $i, j, k, l$, and all other unlinked products vanish. The old unit element $c$ has $P(c) y=y^{j}$, so $c$ remains the unit only if $j=I$ is trivial. Thus the algebra case $H_{n}\left(D, D_{0}\right)$ results from choosing the trivial automorphism $j$. If $j$ is not an inner automorphism on $J$, i.e., $x^{j}$ is not of the form $U(u) x$, then $H_{n}\left(D, D_{0}\right)$ is not a Jordan algebra: there is no unit element $u$, since $P(u)=U(u) P(c)=$ $I$ iff $U(u)=P(c)=j$.

1. Compatible tripotents. An element $e \in J$ is tripotent if $P(e) e=e ;$ such an element determines a decomposition $I=E_{1} \oplus E_{0} \oplus$ $E_{0}$ of the identity operator on $J$ into a direct sum of Peirce projection operators

$$
\begin{aligned}
& E_{2}(e)=P(e)^{2}, \quad E_{1}(e)=L(e, e)-2 P(e)^{2}, \\
& E_{0}(e)=B(e, e)=I-L(e, e)+P(e)^{2} .
\end{aligned}
$$

Such an operator decomposition leads immediately to a Peirce decomposition

$$
J=J_{2} \oplus J_{1} \oplus J_{0} \quad\left(J_{i}=J_{i}(e)=E_{i}(e) J\right)
$$

of the underlying space $J$. (Following Loos, we use as indices the eigenvalues $2,1,0$ of $L(e, e)$ rather than the indices $1,1 / 2,0)$. The Peirce spaces are sub-triple systems characterized by

$$
\begin{aligned}
& J_{0}(e)=\{x \mid L(e, e) x=P(e) x=0\} \\
& J_{1}(e)=\{x \mid L(e, e) x=x, P(e) x=0\} \\
& J_{2}(e)=\{x \mid L(e, e) x=2 x, P(e) x=\bar{x}, \overline{\bar{x}}=x\} .
\end{aligned}
$$

A peculiarity of triples is that $P(e)$ is not the identity on $J_{2}(e)$ but merely an involution $x \rightarrow \bar{x}$. The Peirce spaces multiply according to $P\left(J_{i}\right) J_{j} \subset J_{2 i-j},\left\{J_{i} J_{j} J_{k}\right\} \subset J_{i-j+k}$, or more specifically for $i=2,0, j=$ $2-i$

$$
\begin{aligned}
& P\left(J_{i}\right) J_{j}=\left\{J J_{i} J_{j}\right\}=0, P\left(J_{i}\right) J_{i} \subset J_{i} \\
& P\left(J_{i}\right) J_{1}=0, P\left(J_{1}\right) J_{i} \subset J_{j},\left\{J_{0} J_{1} J_{2}\right\} \subset J_{1} \\
& \left\{J_{1} J_{1} J_{i}\right\} \subset J_{i},\left\{J_{i} J_{i} J_{1}\right\} \subset J_{1}, P\left(J_{1}\right) J_{1} \subset J_{1} .
\end{aligned}
$$

We will make frequent use of the fact that multiplications $L(x, y)$ by elements in the same Peirce space leave all Peirce spaces invariant,

$$
L\left(x_{i}, y_{i}\right) J_{j} \subset J_{j}
$$

We also have the general rules 


$$
L\left(x_{2}, e\right)=L\left(e, \bar{x}_{2}\right), \quad P\left(x_{2}\right) P(e)=P(e) P\left(\bar{x}_{2}\right) \quad\left(x_{2} \in J_{2}(e)\right) .
$$

(See [2], [4] for results on Peirce decompositions.)

If $e$ and $f$ are orthogonal tripotents, the corresponding Peirce projections commute and yield a double Peirce decomposition of the space. However, $e$ and $f$ by no means need be orthogonal in order for this double decomposition to exist: all that is necessary is that $e$ and $f$ be compatible in the sense that the corresponding Peirce projections commute,

$$
\left[E_{i}(e), E_{j}(f)\right]=0 \quad(i, j=0,1,2) .
$$

We can describe rather briefly the condition that two tripotents be compatible; it is very important that this depends only on the tripotents themselves, and not on the triple system in which they are imbedded.

1.6. Compatibility Criterion. Two tripotents $e, f$ are compatible iff $\{e e f\}$ lies in $J_{2}(f)$, in which case it is symmetric under the involution $P(f)$ of $J_{2}(f)$ :

$$
\{e e f\}=P(f)\{e e f\} .
$$

Proof. First let us show this condition is symmetric in $e$ and $f$, i.e., it implies $\{f f e\} \in J_{2}(e)$. For arbitrary tripotents $e, f$, if we write $x=\{e e f\}$ in terms of its Peirce components $x=x_{2}+x_{1}+x_{0}$ for $x_{i} \in J_{i}(f)$ we have $2 x_{2}+x_{1}=L(f, f) x=\{f f\{e e f\}\}=\{L(e, e) P(f)+$ $P(f) L(e, e)\} f($ by $(0.4))=L(e, e) f+P(f) L(e, e) f=\left(x_{2}+x_{1}+x_{0}\right)+\bar{x}_{2}$. Thus always $x_{0}=0$ and always $x_{2}$ is symmetric,

$$
\{e e f\}=x_{2}+x_{1}, \quad x_{i} \in J_{i}(f), \quad x_{2}=\bar{x}_{2}=P(f) x_{2} .
$$

The condition $\{e e f\} \in J_{2}$ is just that $x_{1}=0$. Now assume $\{e e f\} \in J_{2}(f)$, i.e., $x_{1}=0$ and $\{e e f\}=x_{2}$; then $P(e)\{f f e\}=P(e) L(e, f) f=\{-P(f, e) L(e, e)+$ $P(P(e) e, f)+P(P(e, f) e, e)\} f$ (by linearized $(0.2))=-\left\{f x_{2} e\right\}+\{e f f\}+$ $\left\{x_{2} f e\right\}=\{f f e\}$ from (1.4) since $\bar{x}_{2}=x_{2}$ by (1.7). Thus $\{f f e\}=P(e)\{f f e\} \in$ $J_{2}(e)$ and the condition is symmetric in $e$ and $f$.

Now we show the condition $\{e e f\} \in J_{2}(f)$ (and its consequences $\left.\{f f e\} \in J_{2}(e)\right)$ are necessary and sufficient for compatibility (1.5). Certainly they are necessary: $L(e, e) f \in L(e, e) J_{2}(f)=\left\{E_{1}(e)+\right.$ $\left.2 E_{2}(e)\right\} E_{2}(f) J=E_{2}(f)\left\{E_{1}(e)+2 E_{2}(e)\right\} J \subset J_{2}(f)$ by (1.1) and commutativity. The hard part is showing sufficiency. Since the Peirce projections $E_{i}(e)$ of (1.1) are linear combinations of $L(e, e), P(e)^{2}$, and $I$ it suffices to prove

(i ) $[L(e, e), L(f, f)]=0$

(ii) $\left[L(e, e), P(f)^{2}\right]=\left[L(f, f), P(e)^{2}\right]=0$ 
(iii) $\left[P(e)^{2}, P(f)^{2}\right]=0$.

Furthermore, by symmetry in $e$ and $f$ we need only prove the first part of (ii).

For (i) we have $[L(e, e), L(f, f)]=L(\{e e f\}, f)-L(f,\{e e f\})$ (by $(0.5))=L\left(x_{2}, f\right)-L\left(f, x_{2}\right)=L\left(f, \bar{x}_{2}\right)-L\left(f, x_{2}\right)=0$ by (1.4), (1.7), and the hypothesis $\{e e f\}=x_{2}$. We remark that if $1 / 2 \in \Phi$ then (i) already yields (ii), (iii) since $2 P(f)^{2}=L(f, f)^{2}-L(f, f)$ is generated by $L(f, f)$ according to $(0.6)$.

In general, for (ii) we compute $\left[L(e, e), P(f)^{2}\right]=\{L(e, e) P(f)\} P(f)-$ $P(f)\{P(f) L(e, e)\}=\{P(\{e e f\}, f)-P(f) L(e, e)\} P(f)-P(f)\{P(\{e e f\}, f)-$ $L(e, e) P(f)\} \quad($ by $(0.4))=P\left(x_{2}, f\right) P(f)-P(f) P\left(x_{2}, f\right)=P(f) P\left(\bar{x}_{2}, f\right)-$ $P(f) P\left(x_{2}, f\right)=0$ from (1.4) and (1.7).

Before considering (iii) we pause to establish

(iv) $P(e)^{2} f=z_{2} \in J_{2}(f)$

(v) $L(e, e) x_{2}=x_{2}+2 z_{2}$

(vi) $P\left(x_{2}\right) f=z_{2}+\bar{z}_{2}+x_{2}$.

By (ii) $P(e)^{2}$ commutes with $L(f, f)$ and hence leaves its 2-eigenspace invariant: $\{L(f, f)-2\} f=0 \Rightarrow\{L(f, f)-2\} P(e)^{2} f=0 \Rightarrow z=P(e)^{2} f=$ $z_{2}+z_{0}$ for $2 z_{0}=0$. Hence $L(e, e) x_{2}=L(e, e)^{2} f=\left\{L(e, e)+2 P(e)^{2}\right\} f$ (by $(0.6))=x_{2}+2\left(z_{2}+z_{0}\right)=x_{2}+2 z_{2}$ as in $(\mathrm{v})$. On the other hand, identifying Peirce components in $0=\left\{P(\{e e f\})+P\left(P(e)^{2} f, f\right)-\right.$ $\left.P(e)^{2} P(f)-P(f) P(e)^{2}-L(e, e) P(f) L(e, e)\right\} f($ by $(0.8))=P\left(x_{2}\right) f+\{z f f\}-$ $z-P(f) z-L(e, e) \bar{x}_{2}=P\left(x_{2}\right) f+2 z_{2}-\left(z_{2}+z_{0}\right)-\bar{z}_{2}-\left(x_{2}+2 z_{2}\right) \quad$ (by $(\mathrm{v}))=P\left(x_{2}\right) f-\left(z_{2}+\bar{z}_{2}+x_{2}\right)-z_{0}$ yields $z_{0}=0$, so $z=z_{2} \in J_{2}(f)$ as in (iv), and $P\left(x_{2}\right) f=z_{2}+\bar{z}_{2}+x_{2}$ as in (vi).

Finally we are ready to establish (iii).

$\left[P(e)^{2}, P(f)^{2}\right]=\left\{P(e)^{2} P(f)\right\} P(f)-P(f)\left\{P(f) P(e)^{2}\right\}=\{P(\{e e f\})+$ $\left.P\left(P(e)^{2} f, f\right)-P(f) P(e)^{2}-L(e, e) P(f) L(e, e)\right\} P(f)-P(f)\{P(\{e e f\})+$ $\left.P\left(P(e)^{2} f, f\right)-P(e)^{2} P(f)-L(e, e) P(f) L(e, e)\right\}($ by $(0.8))=\left[P\left(x_{2}\right), P(f)\right]+$ $\left[P\left(z_{2}, f\right), P(f)\right]-[L(e, e) P(f) L(e, e), P(f)]$ (using (iv)) $=P(f)\left\{P\left(\bar{x}_{2}\right)-\right.$ $\left.P\left(x_{2}\right)+P\left(\bar{z}_{2}, f\right)-P\left(z_{2}, f\right)\right\}-P\left(x_{2}, f\right) L(e, e) P(f)+P(f) L(e, e) P\left(x_{2}, f\right)$ (using (1.4) and (0.4)) $=P(f) P\left(\bar{z}_{2}-z_{2}, f\right)-P\left(x_{2}, f\right)\left\{P\left(x_{2}, f\right)-P(f) L(e, e)\right\}+$ $P(f)\left\{P\left(\left\{e e x_{2}\right\}, f\right)+P\left(x_{2},\{e e f\}\right)-P\left(x_{2}, f\right) L(e, e)\right\}$ (by (1.7) and $\left.(0.4)\right)=$ $P(f) P\left(\bar{z}_{2}-z_{2}, f\right)-P\left(x_{2}, f\right)^{2}+P(f)\left\{P\left(x_{2}+2 z_{2}, f\right)+P\left(x_{2}, x_{2}\right)\right\}+$ $\left[P\left(x_{2}, f\right), P(f)\right] L(e, e)$ (using (v) and $\left.(1.7)\right)=P(f)\left\{P\left(\bar{z}_{2}+z_{2}+x_{2}, f\right)-\right.$ $\left.P(f) L\left(x_{2} f\right) L\left(f, x_{2}\right)+2 P\left(x_{2}\right)\right\}+0$ (using (1.4), (1.7), and noting by linearized (0.6) that $P\left(x_{2}, f\right)^{2}=L\left(x_{2}, x_{2}\right) L(f, f)+L\left(x_{2}, f\right) L\left(f, x_{2}\right)-L\left(x_{2}\right.$, $\left.\left\{x_{2} f f\right\}\right)=L\left(x_{2}, x_{2}\right)\{L(f, f)-2 I\}+L\left(x_{2}, f\right) L\left(f, x_{2}\right)$, yet $P\left(x_{2}, f\right)^{2}$ lives on $J_{2}(f)$ where $L(f, f)=2 I$, so $P\left(x_{2}, f\right)^{2}=E_{2} P\left(x_{2}, f\right)^{2}=P(f)^{2} L\left(x_{2}, f\right) L\left(f, x_{2}\right)$ on $J)=P(f)\left\{P\left(P\left(x_{2}\right) f, f\right)-P(f) L\left(x_{2}, f\right) L\left(f, x_{2}\right)+2 P\left(x_{2}\right)\right\}$ (by (vi)) $=$ $P(f)\left\{P\left(x_{2}, f\right) L\left(f, x_{2}\right)-L(f, f) P\left(x_{2}\right)-P\left(P(f) x_{2}, f\right) L\left(f, x_{2}\right)+2 P\left(x_{2}\right)\right\}$ (by $(0.7),(0.2))=0$ since $L(f, f)=2 I$ on $J_{2}(f)$ and $P(f) x_{2}=\bar{x}_{2}=x_{2}$ by (1.7). 
CoRollary 1.8. Tripotents e, $f$ are compatible iff

$$
f=f_{2}+f_{1}+f_{0} \text { for elements } f_{i} \in J_{i}(e) \cap J_{2}(f) .
$$

This will be the case under any of the following conditions:

(i ) e, f are orthogonal: $P(e) f=P(f) e=L(e, e) f=L(f, f) e=0$

(ii) $e, f$ are collinear: $P(e) f=P(f) e=0, L(e, e) f=f, L(f, f) e=e$

(iii) one lies in a single Peirce space of the other, $f \in J_{i}(e)$ for $i=2,1,0$

(iv) $f=f_{2}+f_{1}+f_{0}$ for orthogonal tripotents $f_{i} \in J_{i}(e)$.

Proof. If $e, f$ are compatible the Peirce $i$-component $f_{i}=E_{i}(e) f$ of $f$ remains in $J_{2}(f)$; conversely, if $f_{i} \in J_{i}(e) \cap J_{2}(f)$ then $\{e e f\}=$ $2 f_{2}+f_{1} \in J_{2}(f)$. (i)-(iii) are special cases: (i) $f \in J_{0}(e)$, (ii) $f \in J_{1}(e)$, (iii) $f \in J_{2}(e)$. For (iv): $\{f f e\}=\sum\left\{f_{i} f_{i} e\right\}$ (by orthogonality $\left.f_{i} \perp f_{j}\right) \in J_{2}(e)$ by (1.3).

REMARK 1.9. The condition that an element $f=a_{2}+a_{1}+a_{0}$ $\left(a_{i} \in J_{i}(e)\right)$ be tripotent is

$$
\begin{aligned}
& a_{2}=P\left(a_{2}\right) a_{2}+P\left(a_{1}\right) a_{0}+\left\{a_{1} a_{1} a_{2}\right\} \\
& a_{1}=P\left(a_{1}\right) a_{1}+\left\{a_{0} a_{1} a_{2}\right\}+\left\{a_{0} a_{0} a_{1}\right\}+\left\{a_{2} a_{2} a_{1}\right\} \\
& a_{0}=P\left(a_{0}\right) a_{0}+P\left(a_{1}\right) a_{2}+\left\{a_{1} a_{1} a_{0}\right\} .
\end{aligned}
$$

For such an $f$, the compatibility condition $\{f f e\} \in J_{2}(e)$ reduces by (1.3) to

$$
\left\{a_{0} a_{1} e\right\}+\left\{a_{1} a_{2} e\right\}=0
$$

in which case

$$
\begin{aligned}
& \left\{a_{2} a_{2} a_{1}\right\}=\left\{a_{0} a_{0} a_{1}\right\}=-\left\{a_{0} a_{1} a_{2}\right\} \\
& \left\{a_{1} a_{1} a_{0}\right\}=-2 P\left(a_{1}\right) a_{2},\left\{a_{1} a_{1} a_{2}\right\}=-2 P\left(a_{1}\right) a_{0}
\end{aligned}
$$

so the tripotence condition becomes

$$
\begin{aligned}
& a_{2}=P\left(a_{2}\right) a_{2}-P\left(a_{1}\right) a_{0} \\
& a_{1}=P\left(a_{1}\right) a_{1}-\left\{a_{0} a_{1} a_{2}\right\} \\
& a_{0}=P\left(a_{0}\right) a_{0}-P\left(a_{1}\right) a_{2} .
\end{aligned}
$$

From this it is easy to see that if $a_{2}=0$ then $f=a_{1} \oplus a_{0}$ is the direct sum of two orthogonal tripotents, similarly if $a_{1}=0$ or $a_{0}=$ 0 . Thus a compatible $f$ is not too far away from being a direct sum of orthogonal tripotents $f_{i} \in J_{i}(e)$.

If $J$ is a Jordan algebra instead of a triple system and $e, f$ are idempotents $\left(e^{2}=e, f^{2}=f\right)$ instead of merely tripotents, then com- 
patibility reduces to

1.13. Compatibility CRIterion For IDempotents. Two idempotents e, $f$ in a Jordan algebra are compatible iff $f=f_{2} \oplus f_{0}$ for orthogonal idempotents $f_{i} \in J_{i}(e)$.

Proof. The condition that $f=a_{2}+a_{1}+a_{0}$ is idempotent is

$$
\begin{aligned}
& a_{2}=a_{2}^{2}+E_{2}(e) a_{1}^{2} \\
& a_{1}=\left(a_{2}+a_{0}\right) \circ a_{1} \\
& a_{0}=a_{0}^{2}+E_{0}(e) a_{0}^{2}
\end{aligned}
$$

and compatibility (1.11) becomes

$$
a_{0} \circ a_{1}+a_{1} \circ a_{2}=0,
$$

hence $a_{1}=0$ and $a_{2}=a_{2}^{2}, a_{0}=a_{0}^{2}$ are orthogonal idempotents. Conversely, if $f=f_{2}+f_{0}$ then $e, f$ are compatible by (1.8iv).

Note that the strong compatibility condition that the operators $P(e), L(e, e)$ commute with $P(f), L(f, f)$ (not merely $P(e)^{2}$ and $\left.P(f)^{2}\right)$ is not an intrinsic condition: it depends on how $e, f$ are imbedded in $J$. For example, $e=1[12]$ and $f=1$ [13] are collinear and strongly compatible in $D[12]+D[13] \cong M_{1,2}(D)$, but not in $H_{3}(D)$ since $P(e) P(f)$ $1[33]=P(e) 1[11]=1[22] \neq 0=P(f) P(e) 1[33]$.

The most important examples of compatible tripotents are either orthogonal $e \perp f$ (each lies in the 0 -space of the other) or collinear $e T f$ (each lies in the 1-space of the other). In the remainder of this section we investigate what collinearity amounts to in basic examples of triple systems. Recall that tripotents $e, f$ are collinear if $P(e) f=P(f) e=0, L(e, e) f=f, L(f, f) e=e$.

Let us note that in a Jordan algebra we cannot have collinear idempotents; collinearity is strictly for tripotents.

Proposition 1.14. Two nonzero idempotents in a Jordan algebra can never be collinear.

Proof. If $e \in J_{1}(f)$ and $f \in J_{1}(e)$ are idempotents then $f=\{e e f\}=$ $e^{2} \circ f=e \circ f=e \circ f^{2}=\{e f f\}=e$, so $f=P(f) f=P(f) e=0$ and dually $e=0$. (Alternately, if $f \in J_{1}(e)$ then $f^{2} \in J_{2}(e)+J_{0}(e)$, so the only idempotent in $J_{1}(e)$ is $f=0$. Or yet again, the result follows directly from (1.13).) 


\section{Collinearity in $J T(A)$}

From any associative algebra $A$ we can form a Jordan triple system $J T(A)$ on the linear space $A$ by

$$
P(x) y=x y x .
$$

Here an element $x$ is tripotent iff $x x x=x$, i.e., $x^{8}=x$. In this case $e=x^{2}$ is an ordinary associative idempotent, and $e x=x e=x$. Thus $x$ lies in the unital Peirce subalgebra $e A e$ and is a "square root of unity" therein. Examples of collinear tripotents are the matrix units $x=E_{12}, y=E_{13}$ or $x=E_{12}+E_{21}, y=E_{13}+E_{31}$. The latter example is quite general, since we have

1.15. CollineARITY THEOREM FOR $J T(A)$. Two nonzero tripotents $x, y$ in $J T(A)$ are collinear iff there is a subalgebra $B \cong M_{3}(\Phi)$ of $A$ with $x \cong E_{12}+E_{21}, y \cong E_{13}+E_{31}$.

Proof. Tripotence means $x^{3}=x, y^{3}=y$ and collinearity means $x y x=y x y=0, x^{2} y+y x^{2}=y, y^{2} x+x y^{2}=x$. Then $x^{2} y^{2}=\left(y-y x^{2}\right) y=$ $y\left(y-x^{2} y\right)=y^{2} x^{2}$, so a direct calculation shows

$$
\begin{aligned}
& e_{11}=x^{2} y^{2}=y^{2} x^{2} \quad e_{22}=x y^{2} x \quad e_{33}=y x^{2} y \\
& e_{12}=y^{2} x \quad e_{21}=x y^{2} \quad e_{13}=x^{2} y \quad e_{31}=y x^{2} \quad e_{23}=x y \quad e_{32}=y x
\end{aligned}
$$

form a complete family of matrix units, hence yield an isomorphism of $M_{3}(\Phi)$ into $A$ by $E_{i j} \rightarrow e_{i j}$, with $x=e_{12}+e_{21}, y=e_{13}+e_{31}$.

\section{Collinearity in $J T(J)$}

Generalizing the previous example, if $J$ is any Jordan algebra we obtain a Jordan triple system $J T(J)$ by forgetting the squaring operation:

$$
P(x) y=U(x) y \text {. }
$$

In a Jordan algebra we define an element $x$ to be strictly tripotent if it "strictly" satisfies the relation $x^{3}=x$, i.e.,

$$
x^{3}=x, x^{4}=x^{2} .
$$

Thus there is a distinction between $x$ being strictly tripotent in the Jordan algebra $J$ as in (1.16), and merely being tripotent $x^{3}=x$ in the Jordan triple system $J T(J)$. The two notions coincide if $J$ is special or nondegenerate or if $1 / 2 \in \Phi$.

LEMMA 1.17. An element $x$ is tripotent in $J T(J)$ iff $x$ lies in $J_{2}(e)$ for an idempotent $e$ with $x^{3}=x, x^{4}=e$. If $J$ has no trivial 
elements $z$ with $2 z=V(z)=U(z)=0$ (e.g., if $1 / 2 \in \Phi$ or $J$ is nondegenerate), or if $J$ is special, then $x^{3}=x$ implies $x^{4}=x^{2}$, so all tripotents are strict.

Proof. Always $x^{3}=x$ implies $\left(x^{4}\right)^{2}=U(x)^{3} x^{2}=U(x) x^{2}=x^{4}$, so $x^{4}=e$ is idempotent with $U(e)=U(x)^{4}=U(x)^{2}$ so that $U(e) x=x$. If $J$ is special, $J \subset A^{+}$, then $x^{3}=x$ implies $x x^{3}=x x$, i.e., $x^{4}=x^{2}$. In the general case there is no "left multiplication by $x$ " (though $1 / 2 V(x)$ works when $1 / 2 \in \Phi)$. The element $z=x^{4}-x^{2}$ may not be zero, but it has

$$
\begin{aligned}
& 2 z=2 x^{4}-2 x^{2}=x \circ\left(x^{3}-x\right)=0 \\
& V(z)=V\left(x^{4}-x^{2}\right)=V\left(x, x^{3}-x\right)=0 \\
& U(z)=U\left(x^{4}-x^{2}\right)=U(x) U\left(x^{3}-x\right)=0 .
\end{aligned}
$$

Thus when $J$ has no such trivial $z$ we have $z=0$ and $x^{4}=x^{2}$.

REMARK 1.18. When $x^{3}=x$ we do not always have $z=0$, as the example $J=\Phi[x] / K$ shows for $\Phi[x]$ the polynomial ring in one indeterminate and $K$ is the Jordan ideal spanned by $x-x^{3}, 2 x^{2}-$ $2 x^{4}, x^{i}-x^{j}$ for $i \equiv j \bmod 4$. Here $J$ is spanned by $1=e, x, z$ with $x^{2}=1+z, x^{3}=x, x^{4}=1,2 z=0$, but $z \neq 0$ if $1 / 2 \notin \Phi$.

An example of collinear tripotents in the Jordan matrix algebra $H_{n}(D)$ of Hermitian $n \times n$ matrices over $D$ is $x=1[12], y=1$ [13]. This example is in fact typical.

1.19. COLLINEARITY THEOREM FOR J. Two nonzero strict tripotents $x, y$ in a Jordan algebra $J$ are collinear iff there is a subalgebra $B \cong H_{3}(\Phi)$ with $x \cong 1[12]=E_{12}+E_{21}, y \cong 1[13]=E_{13}+E_{31}$.

Proof. The condition is clearly sufficient. To see it is necessary, note that strict tripotence in means $x^{3}=x, x^{4}=x^{2}, y^{3}=y, y^{4}=y^{2}$ and collinearity means $U(x) y=U(y) x=0, \quad x^{2} \circ y=y, \quad y^{2} \circ x=x$. From (0.8), (0.4), (0.6) we get $U(x)=U(\{y y x\})=U(x) U(y)^{2}+$ $U(y)^{2} U(x)+V(y, y) U(x) V(y, y)=U(x) U(y)^{2}+U(y)^{2} U(x)+V(y, y)\{-V(y$, $y)+2 U(x)\}=U(x) U(y)^{2}+U(y)^{2} U(x)+\left\{-2 U(y)^{2}+V(y, y)\right\} U(x)=$ $U(x) U(y)^{2}-U(y)^{2} U(x)+V(y, y) U(x)$ and similarly $U(x)=-U(x) U(y)^{2}+$ $U(y)^{2} U(x)+U(x) V(y, y)$, so $\left[U\left(x^{2}\right), U\left(y^{2}\right)\right]=U(x)\left[U(x), U\left(y^{2}\right)\right]+[U(x)$, $\left.U\left(y^{2}\right)\right] U(x)=U(x)\{U(x)-V(y, y) U(x)\}+\{U(x) V(y, y)-U(x)\} U(x)=0$, hence $U\left(x^{2}\right) y^{4}-U\left(y^{2}\right) x^{4}=U\left(x^{2}\right) U\left(y^{2}\right) 1-U\left(y^{2}\right) U\left(x^{2}\right) 1=0$ and $U\left(x^{2}\right) y^{2}=$ $U\left(y^{2}\right) x^{2}$ by (1.16). Then a calculation analogous to (1.15) shows

$$
\begin{aligned}
& e_{1}=U_{x^{2}} y^{2}=U_{y^{2} x^{2}} \quad e_{2}=U_{x} y^{2} \quad e_{3}=U_{y} x^{2} \\
& u_{12}=x \quad u_{13}=y \quad u_{23}=x \circ y
\end{aligned}
$$


forms a family of hermitian matrix units and thus yields an imbedding $H_{3}(\Phi) \rightarrow J$ sending $1[i i] \rightarrow e_{i}, 1[i j] \rightarrow u_{i j}$.

\section{Collinearity in $J T(A, *)$}

A more general method for obtaining Jordan triples $T$ from Jordan algebras $J$ is through $P(x) y=U(x) y$ * for some involution * of $J$. However, there seems to be no relation between tripotents $x \in J T(J, *)$ and idempotents in $J$ (in general there don't seem to be idempotents in $J$ ). In the special case where $J=A^{+}$, so $P(x) y=$ $x y^{*} x$, an element $x$ is tripotent iff $x=a+b$ for $a \in e A e, b \in e A(1-e)$ satisfying $a a^{*}+b b^{*}=e$ for a symmetric idempotent $e$ (namely $e=$ $x x^{*}, a=x e, b=x(1-e)$ ). Collinearity becomes complicated,

1.20. Collinearity Theorem for $J T(A, *)$. Two nonzero tripotents $x, y$ in $J T(A, *)$ are collinear iff there are two families $e_{11}$, $e_{22}, e_{33}$ and $f_{11}, f_{22}, f_{33}$ of symmetric orthogonal idempotents and elements $x_{i j}, y_{i j}$ in $e_{i i} A f_{j j}$ such that

$$
\begin{aligned}
& x=x_{12}+x_{21}, \quad y=y_{13}+y_{31} \\
& x_{12} x_{12}^{*}=y_{13} y_{13}^{*}=e_{11}, \quad x_{21} x_{21}^{*}=e_{22}, \quad y_{31} y_{31}^{*}=e_{33} \\
& x_{21}^{*} x_{21}=y_{31}^{*} y_{31}=f_{11}, \quad x_{12}^{*} x_{12}=f_{22}, \quad y_{13}^{*} y_{13}=f_{33} \text {. }
\end{aligned}
$$

Proof. $\quad a \rightarrow a^{\prime}=\left(\begin{array}{cc}0 & a \\ a^{*} & 0\end{array}\right)$ imbeds $J T(A, *)$ in $J T(B)$ for $B=M_{2}(A)$, so from (1.15) $x^{\prime}=e_{12}^{\prime}+e_{21}^{\prime}, y^{\prime}=e_{13}^{\prime}+e_{31}^{\prime}$ for $e_{i j}^{\prime}=\left(\begin{array}{cc}0 & x_{i j} \\ x_{j i}^{*} & 0\end{array}\right)(i, j=1,2)$ $e_{i k}^{\prime}=\left(\begin{array}{cc}0 & y_{i k} \\ y_{i k}^{*} & 0\end{array}\right)(i, k=1,3), e_{i i}^{\prime}=\left(\begin{array}{cc}e_{i} & 0 \\ 0 & f_{i}\end{array}\right)$ we get the result.

2. Compatible Peirce decomposition. A finite family $\mathscr{E}=$ $\left\{e_{1}, \cdots, e_{n}\right\}$ of tripotents is compatible if every pair $e_{i}, e_{j}$ is compatible. Now any time we have a finite number of commuting decompositions $I=E_{2}\left(e_{i}\right)+E_{1}\left(e_{i}\right)+E_{0}\left(e_{i}\right)$ relative to $e_{1}, \cdots, e_{n}$ we can put them together to get a simultaneous decomposition

$$
I=\prod_{i=1}^{n}\left\{E_{2}\left(e_{i}\right)+E_{1}\left(e_{i}\right)+E_{0}\left(e_{i}\right)\right\}=\sum_{\left\langle i_{1}, \cdots, i_{n}\right\} \in\{2,1,0\}} E_{\left\langle i_{1}, \cdots, i_{n}\right)}
$$

of the identity operator for

$$
E_{\left(i_{1}, \cdots, i_{n}\right)}=E_{i_{1}}\left(e_{1}\right) E_{i_{2}}\left(e_{2}\right) \cdots E_{i_{n}}\left(e_{n}\right) .
$$

By commutativity these $E$ 's are supplementary projection operators, and hence yield a compatible Peirce decomposition.

$$
\begin{aligned}
& J=\bigoplus_{\left(i_{1}, \cdots, i_{n}\right) \in\{2,1,0 ; n} J_{\left(i_{1}, \cdots, i_{n}\right)} \\
& J_{\left(i_{1}, \cdots, i_{n}\right)}=E_{\left(i_{1}, \cdots, i_{n}\right)} J=E_{i_{1}}\left(e_{1}\right) \cdots E_{i_{n}}\left(e_{n}\right) J=J_{i_{1}}\left(e_{1}\right) \cap \cdots \cap J_{i_{n}}\left(e_{n}\right)
\end{aligned}
$$


of the underlying space $J$. We retain the parentheses in the subscripts to distinguish them from the standard orthogonal Peirce decompositions.

WARNING. The labelling of mixed Peirce spaces IS NOT SYMMETRIC IN THE INDICES $i_{1}, \cdots, i_{n}$; it depends on an ordering $e_{1}, \cdots, e_{n}$ of the compatible tripotents. It therefore differs from the usual labelling in the case of two orthogonal tripotents. Indeed, if $e_{1}, e_{2}$ are orthogonal the above 9-term decomposition $J=\sum_{i, j=2,1,0} J_{(i j)}$ reduces to a 6 -term decomposition since

$$
J_{(22)}=J_{(21)}=J_{(12)}=0,
$$

and

$$
\begin{gathered}
J=J_{(20)} \oplus J_{(11)} \oplus J_{(02)} \oplus J_{(10)} \oplus J_{(01)} \oplus J_{(00)} \\
\left(J_{(i j)}=J_{i}\left(e_{1}\right) \cap J_{j}\left(e_{2}\right)\right)
\end{gathered}
$$

is usually written as

$$
J=J_{11} \oplus J_{12} \oplus J_{22} \oplus J_{10} \oplus J_{20} \oplus J_{00} \quad\left(J_{i i}=J_{2}\left(e_{i}\right), J_{i j}=J_{j i}\right) .
$$

It must be emphasized that such a $3^{n}$-term mixed Peirce decomposition relative to compatible $e_{1}, \cdots, e_{n}$ is much more complicated than the $1 / 2(n+1)(n+2)$-term decomposition relative to orthogonal $e_{1}, \cdots, e_{n}$. The usual philosophy behind Peirce decompositions is to reduce the abstract product on $J$ to more tractable products between the individual Peirce spaces $J_{i}$. In the case of mixed Peirce decompositions, however, the product rules for the Peirce spaces are simply those of the individual tripotents (e.g., $P\left(J_{(210)}\right) J_{(220)} \subset J_{(200)}$ since $P\left(J_{2}\left(e_{1}\right)\right) J_{2}\left(e_{1}\right) \subset J_{2}\left(e_{1}\right), P\left(J_{1}\left(e_{2}\right)\right) J_{2}\left(e_{2}\right) \subset J_{0}\left(e_{2}\right), P\left(J_{0}\left(e_{3}\right)\right) J_{0}\left(e_{3}\right) \subset J_{0}\left(e_{3}\right)$ by (1.2)). There is almost no relation between the Peirce decompositions since there is almost no relation between the tripotents.

We seldom want to consider all terms of a mixed Peirce decomposition individually. For many purposes a very crude decomposition $J=J_{2} \oplus J_{1} \oplus J_{0}$ of $J$ suffices, where $J_{2}$ is the part "covered" by the $e_{i}$ 's (the part where they act, in concert, like a unit), $J_{1}$ is the part "half-covered" by the $e_{i}$ 's and $J_{0}$ is orthogonal to the $e_{i}$ 's.

2.2. Peirce Decomposition Relative to a Compatible Family. If $\mathscr{E}=\left\{e_{1}, \cdots, e_{n}\right\}$ is a compatible family of tripotents in a Jordan triple system $J$, there is a Peirce decomposition

$$
J=J_{2}(\mathscr{E}) \oplus J_{1}(\mathscr{E}) \oplus J_{0}(\mathscr{E})
$$

for

$$
J_{2}(\mathscr{E})=\sum_{i} J_{2}\left(e_{i}\right)=\sum J_{\left(i_{1}, \cdots, 2, \cdots, i_{\mathfrak{m}}\right)}
$$




$$
\begin{aligned}
& J_{1}(\mathscr{E})=\sum_{i} J_{1}\left(e_{i}\right) \cap \bigcap_{j \neq i}\left\{J_{0}\left(e_{j}\right)+J_{1}\left(e_{j}\right)\right\}=\sum_{i_{r} \in\{1,0\}} J_{\left(i_{1}, \cdots, 1, \cdots, i_{n}\right)} \\
& J_{0}(\mathscr{E})=\bigcap_{i} J_{0}\left(e_{i}\right)=J_{(0, \cdots, 0)} .
\end{aligned}
$$

These spaces multiply according to the orthogonality rules

(P1) $P\left(J_{0}\right) J_{2}=P\left(J_{2}\right) J_{0}=\left\{J_{0} J_{2} J\right\}=\left\{J_{2} J_{0} J\right\}=P\left(J_{0}\right) J_{1}=0$ ，

(P2) $P\left(J_{0}\right) J_{0} \subset J_{0},\left\{J_{0} J_{0} J_{1}\right\} \subset J_{1},\left\{J_{2} J_{1} J_{0}\right\} \subset J_{1},\left\{J_{1} J_{1} J_{0}\right\} \subset J_{1}+J_{0}$

(P3) $P\left(J_{2}\right) J_{2}+P\left(J_{2}\right) J_{1}+P\left(J_{1}\right) J_{0}+\left\{J_{1} J_{1} J_{2}\right\}+\left\{J_{2} J_{2} J_{1}\right\} \subset J_{2}+J_{1}$,

whereas we can only say $P\left(J_{1}\right) J_{1}$ and $P\left(J_{1}\right) J_{2}$ lie somewhere in $J$.

Proof. Clearly from (2.1), we have a direct decomposition of $J$ into the sum $J_{j}(\mathscr{E})$ of those $J_{\left(i_{1}, \cdots, i_{n}\right)}$ with $(j=2)$ at least one 2, $(j=1)$ at least one 1 but no 2 's, $(j=0)$ only 0 's. The product rules follow "componentwise" from the rules (1.2) for the individual $e_{i}$ 's. For orthogonality (P1), $P(x) y=\{x y z\}=0$ if one of $x, y$ is from $J_{2}(\mathscr{E})$ and the other from $J_{0}(\mathscr{E})$, note the element from $J_{2}$ lies in at least one $J_{2}\left(e_{i}\right)$, and the element from $J_{0}$ lies in all $J_{0}\left(e_{j}\right)$ and hence in particular in $J_{0}\left(e_{i}\right)$, where any product with adjacent terms from $J_{2}\left(e_{i}\right)$ and $J_{0}\left(e_{i}\right)$ vanishes by (1.2). Similarly, if $x, z \in J_{0}, y \in J_{1}$ then $y$ lies in some $J_{1}\left(e_{i}\right)$ and $x$ and $z$ both lie in $J_{0}\left(e_{i}\right)$ so that $P(x) y$ and $\{x y z\}$ lie in $P\left(J_{0}\left(e_{i}\right)\right) J_{1}\left(e_{i}\right)=0$.

For (P2), if $x, y$ lie in $J_{0}$ they lie in all $J_{0}\left(e_{i}\right)$ so that $P(x) y$ does too, i.e., lies in $J_{0}$; if $z \in J_{1}$ then $z$ lies in all $J_{1}\left(e_{j}\right)+J_{0}\left(e_{j}\right)$ and in at least one $J_{1}\left(e_{i}\right)$, so $\{x y z\}$ lies in all $\left\{J_{0}\left(e_{j}\right) J_{0}\left(e_{j}\right)\left(J_{0}\left(e_{j}\right)+J_{1}\left(e_{j}\right)\right)\right\} \subset$ $J_{0}\left(e_{j}\right)+J_{1}\left(e_{j}\right)$ with at least one $J_{1}\left(e_{i}\right)$, i.e., in $J_{1}$. Finally, $\{w z x\}$ in $\left\{J_{2} J_{1} J_{0}\right\}$ or $\left\{J_{1} J_{1} J_{0}\right\}$ has no component in any $J_{2}\left(e_{i}\right)$ since $\left\{J J J_{0}\left(e_{i}\right)\right\} \subset$ $J_{0}\left(e_{i}\right)+J_{1}\left(e_{i}\right)$, and when $w \in J_{2}$ there is no component in $J_{0}$ either since $\left\{J_{2}\left(e_{i}\right) J J\right\} \subset J_{2}\left(e_{i}\right)+J_{1}\left(e_{i}\right)$.

For the relation (P3) we need only show the products $P(x) y$, $\{x y z\}$ have no components in $J_{0}$. This is clear if an external factor $x$ lies in $J_{2}: x$ lies in some $J_{2}\left(e_{i}\right)$, and $\left\{J_{2}\left(e_{i}\right) J J\right\} \subset J_{2}\left(e_{i}\right)+J_{1}\left(e_{i}\right)$. The only product without such external factor from $J_{2}$ is $P\left(J_{1}\right) J_{0}$; but if $x, z \in J_{1}$ and $y \in J_{0}$ then $x$ lies in some $J_{1}\left(e_{i}\right), y$ lies in $J_{0}\left(e_{i}\right)$, and $z$ lies in $J_{1}\left(e_{i}\right)+J_{0}\left(e_{i}\right)$, so $P(x) y \in J_{2}\left(e_{i}\right)$ and $\{x y z\} \in J_{2}\left(e_{i}\right)+J_{1}\left(e_{i}\right)$ has no component in $J_{0}$.

\section{Covering families}

We say a compatible family $\mathscr{E}=\left\{e_{1}, \cdots, e_{n}\right\}$ covers $J$ if $J=$ $J_{2}(\mathscr{E})=\sum J_{2}\left(e_{i}\right)$ is the sum of the various Peirce spaces $J_{2}\left(e_{i}\right)$ where $e_{i}$ acts as unit. $J$ is locally unital if it possesses a compatible covering family $\mathscr{E}$. For example, if $J$ itself has a unit element $e$ (invertible tripotent) then $\mathscr{E}=\{e\}$ is already a covering family. We will see in $\S 3$ that semisimple systems are always locally unital. 
For certain purposes the covering family $\mathscr{E}$ serves just as well as a unit element.

If $J_{i}$ are locally unital with covering families $\mathscr{E}_{i}$, then their direct sum $J=J_{1} \boxplus \cdots \boxplus J_{n}$ is locally unital with covering family $\mathscr{E}=\cup \mathscr{E}_{i}$ (note that $e, f \in \mathscr{E}$ are compatible if they lie in the same $\mathscr{E}_{i}$, and orthogonal-hence compatible-if they lie in different $\mathscr{E}_{i}$, $\mathscr{E}_{j}$ ). If $\mathscr{E}$ covers $J$ and $f: J \rightarrow J^{\prime}$ is a homomorphism, then by (1.6) $f(\mathscr{E})=\left\{f\left(e_{i}\right)\right\}$ remains compatible and covers $f(J) \subset J^{\prime}$, so the image $f(J)$ inherits local unitality. If $K \subset J$ is a subsystem containing $\mathscr{E}, \mathscr{E}$ serves as covering family for $K$ as well. Thus we have

Proposition 2.3. A finite direct sum of locally unital Jordan triple systems is again locally unital. Any homomorphic image of a locally unital system is locally unital. Any subsystem containing the covering family remains locally unital.

As an example, a useful tool in breaking semisimple algebras down into simple ones is the fact that a unital ideal is necessarily a direct summand. The same holds for locally unital triples.

Proposition 2.4. If $K$ is a locally unital ideal in a Jordan triple system $J$, then $K$ is a direct summand:

$$
J=K \boxplus K^{\prime}=J_{2} \boxplus J_{0} \text {. }
$$

Proof. Let $\mathscr{E}=\left\{e_{1}, \cdots, e_{n}\right\}$ be a compatible family of tripotents in $K$ which covers $K$. Since $e_{i} \in K \triangleleft J$ and the Peirce projections $E_{2}\left(e_{i}\right), E_{1}\left(e_{i}\right)$ of (1.1) are multiplications by $e_{i}$ we must have $J_{k}\left(e_{i}\right)=$ $E_{k}\left(e_{i}\right) J \subset K$ for $k=2,1: J_{2}\left(e_{i}\right)+J_{1}\left(e_{i}\right) \subset K$. Summing over all $i$, we get $J_{2}(\mathscr{E})+J_{1}(\mathscr{E}) \subset K$. On the other hand, since $\mathscr{E}$ covers $K$ we have $K=K_{2}(\mathscr{E}) \subset J_{2}(\mathscr{E})$. Thus $J_{1}(\mathscr{E})=0, J_{2}(\mathscr{E})=K$, and $J=K \oplus$ $K^{\prime}$ for $K^{\prime}=J_{0}(\mathscr{E})$. The orthogonality relations (P1) of (2.1) show this is a direct sum of triple systems, hence $J^{\prime}$ is a complementary ideal.

We remark that it is essential here that the family $\mathscr{E}$ be finite: if $J=A^{+}$is the unital Jordan algebra obtained from the associative algebra $A=\Phi I+K$ ( $K$ the row-and-column-finite matrices in $M_{\infty}(\Phi)$ ), then $\mathscr{E}=\left\{E_{1}, E_{2}, \cdots\right\}\left(E_{n}\right.$ the $n \times n$ unit matrix) is a compatible cover of $K$, but $K$ is not an ideal direct summand of $J$.

It is also essential that the family $\mathscr{E}$ be compatible, as the following example shows. Let $J=\Phi e \oplus J_{12} \oplus \Phi f$ be a unital Jordan algebra with unit $1=e+f$, and $J_{12}$ trivial (e.g., if $J \subset M_{2}(\Phi)$ with 
$\left.e=e_{11}, f=e_{22}, J_{12}=\Phi e_{12}\right)$. Then $K=\Phi e+J_{12}$ is an ideal which is not an ideal direct summand, yet it is covered (even spanned) by $e$ and all $e_{i}=e+z_{i}$ for some finite basis $\left\{z_{i}\right\}$ for $J_{12}$. These $e_{i}$ are idempotents but are not compatible with $e$ : $\left\{e e e_{i}\right\}=2 e+z_{i} \notin J_{2}\left(e_{i}\right)$ since $z_{i} \notin J_{2}\left(e_{i}\right)$.

\section{Orthogonal families}

We may regard (2.2) as an analogue for triple systems of the Peirce decomposition relative to a single idempotent $\mathscr{E}$. If we have mutually orthogonal families $\mathscr{E}_{1}, \cdots, \mathscr{E}_{n}$ (for example, if $\mathscr{E}_{i}$ consists of compatible tripotents from $J_{i}$ in a direct sum $J=J_{1} \boxplus \cdots \boxplus J_{n}$ ) we have the following triple system analogue of the Peirce decomposition relative to orthogonal idempotents $\mathscr{E}_{1}, \cdots, \mathscr{E}_{n}$.

2.5. PeirCe DeCOMPosition Relative to ORTHOgONAL COMPATIBLE FAMILIES. If $\mathscr{E}=\mathscr{E}_{1} \cup \cdots \cup \mathscr{E}_{n}$ is the union of mutually orthogonal compatible families $\mathscr{E}_{1}, \cdots, \mathscr{E}_{n}$ of tripotents, then the Jordan triple system $J$ has orthogonal Peirce decomposition

$$
J=\bigoplus_{0 \leqq i, j \leq n} J_{i j} \quad\left(J_{i j}=J_{j i}=J_{i j}(\mathscr{E})\right)
$$

for

$$
\begin{aligned}
& J_{i i}=J_{2}\left(\mathscr{E}_{i}\right)=J_{2}\left(\mathscr{E}_{i}\right) \cap \bigcap_{j \neq i} J_{0}\left(\mathscr{E}_{j}\right) \\
& J_{i j}=J_{1}\left(\mathscr{E}_{i}\right) \cap J_{1}\left(\mathscr{E}_{j}\right)=J_{1}\left(\mathscr{E}_{i}\right) \cap J_{1}\left(\mathscr{E}_{j}\right) \cap \bigcap_{k \neq i, j} J_{0}\left(\mathscr{E}_{k}\right) \\
& J_{i 0}=J_{1}\left(\mathscr{E}_{i}\right) \cap \bigcap_{j \neq i} J_{0}\left(\mathscr{E}_{j}\right) \\
& J_{00}=\bigcap_{i} J_{0}\left(\mathscr{E}_{i}\right) .
\end{aligned}
$$

The Peirce decompositions relative to $\mathscr{E}$ and $\mathscr{E}_{i}$ are recovered by

$$
\begin{aligned}
& J_{2}(\mathscr{E})=\sum_{i=1}^{n} J_{i i}, J_{1}(\mathscr{E})=\sum_{i, j=1}^{n} J_{i j}+\sum_{i=1}^{n} J_{i 0}, J_{0}(\mathscr{E})=J_{00} \\
& J_{2}\left(\mathscr{E}_{i}\right)=J_{i i}, J_{1}\left(\mathscr{E}_{i}\right)=J_{i 0}+\sum_{j \neq i, 0} J_{i j}, J_{0}\left(\mathscr{E}_{i}\right)=\sum_{j, k \neq i} J_{j k} .
\end{aligned}
$$

The Peirce spaces multiply according to the following rules. $A$ product is zero unless its indices can be linked or linked through 0 ,

$$
\begin{gathered}
P\left(J_{i j}\right) J_{k l}=\left\{J_{i j} J_{k l} J_{r s}\right\}=0 \quad \text { if } \quad\{k, l\} \cap\{i, j\}=\varnothing \\
\text { or } \quad\{k, l\} \not \subset\{0, i, j, r, s\}
\end{gathered}
$$

where the only possible nonzero unlinked products are (for $i, j, k, 0 \neq)$

$$
\begin{array}{ll}
\text { (U1) } & P\left(J_{i i}\right) J_{i 0} \subset J_{i i}+J_{i 0} \\
\text { (U2) } & P\left(J_{i j}\right) J_{0 j} \subset J_{i i}+J_{i j}+J_{i 0}
\end{array}
$$


(U3) $\left\{J_{i i} J_{i 0} J_{i k}\right\} \subset J_{i k}$

(U4) $\left\{J_{i j} J_{j 0} J_{j k}\right\} \subset J_{i k}$

while for distinct linked indices $i, j, k, l, 0 \neq$

(P1) $P\left(J_{i i}\right) J_{i i} \subset J_{i i}+J_{i 0}, P\left(J_{00}\right) J_{00} \subset J_{00}$

(P2) $P\left(J_{i j}\right) J_{i i} \subset J_{j j}+J_{j i}+J_{j 0}, P\left(J_{0 j}\right) J_{00} \subset J_{j j}+J_{j 0}$, $P\left(J_{i 0}\right) J_{i i} \subset J_{i i}+J_{i 0}+J_{00}$

(P3) $P\left(J_{i j}\right) J_{i j} \subset J_{i i}+J_{i j}+J_{i 0}+J_{j 0}, P\left(J_{i 0}\right) J_{i 0} \subset J_{i i}+J_{i 0}+J_{00}$

(P4) $\left\{J_{i i} J_{i j} J_{j j}\right\} \subset J_{i j}(j=0$ allowed)

(P5) $\left\{J_{i i} J_{i i} J_{i j}\right\} \subset J_{i j}\left(i=0\right.$ allowed), $\left\{J_{i i} J_{i i} J_{i 0}\right\} \subset J_{i i}+J_{i 0}$

(P6) $\left\{J_{i j} J_{i j} J_{i i}\right\} \subset J_{i i}+J_{i j}+J_{i 0}(j, i=0$ allowed $)$

(P7) $\left\{J_{i i} J_{i j} J_{j k}\right\} \subset J_{i k}\left(j=0\right.$ allowed), $\left\{J_{i i} J_{i j} J_{j 0}\right\} \subset J_{i j}+J_{i 0}$,

$$
\left\{J_{00} J_{0 j} J_{j k}\right\} \subset J_{0 k}
$$

(P8) $\left\{J_{i j} J_{j j} J_{j k}\right\} \subset J_{i k}(j=0$ allowed $),\left\{J_{i j} J_{j j} J_{j 0}\right\} \subset J_{i 0}+J_{i j}$

(P9) $\left\{J_{i j} J_{i j} J_{i k}\right\} \subset J_{i k},\left\{J_{0 j} J_{0 j} J_{0 k}\right\} \subset J_{0 k}+J_{j k},\left\{J_{i 0} J_{i 0} J_{i k}\right\} \subset J_{i k}+J_{0 k}$,

$$
\left\{J_{i j} J_{i j} J_{i 0}\right\} \subset J_{i i}+J_{i j}+J_{i 0}
$$

(P10) $\left\{J_{i j} J_{j k} J_{k i}\right\} \subset J_{i i}+J_{i 0}(j, k=0$ allowed),

$$
\left\{J_{0 j} J_{j k} J_{k 0}\right\} \subset J_{00}+J_{0 j}+J_{0 k}+J_{j k}
$$

(P11) $\left\{J_{i j} J_{j k} J_{k l}\right\} \subset J_{i l}(j, k=0$ allowed $)$

$$
\left\{J_{i j} J_{j k} J_{k 0}\right\} \subset J_{i 0}+J_{i k} \text {. }
$$

Proof. $\mathscr{E}$ is compatible iff each $\mathscr{E}_{i}$ is, since elements from distinct $\mathscr{E}_{i}, \mathscr{E}_{j}$ are orthogonal and hence automatically compatible by (1.8i). We order the tripotents in $\mathscr{E}$ so $\mathscr{E}_{i}<\mathscr{E}_{j}$ if $i<j$ : if $\mathscr{E}_{i}=\left\{e_{i 1}, \cdots, e_{i m}\right\}$ then the indices in $\mathscr{E}=\left\{e_{11}, \cdots, e_{1 m} ; e_{21}, \cdots, e_{2 p} ; \cdots\right.$; $\left.e_{n 1}, \cdots, e_{n q}\right\}$ are arranged in successive ranges corresponding to $\mathscr{E}_{1}, \cdots, \mathscr{E}_{n}$.

The simplifications in the orthogonal Peirce decomposition as compared with (2.1) depend on the well known

LEMMA 2.9. If $e, f$ are orthogonal tripotents in a Jordan triple system then

(i ) $J_{2}(e) \subset J_{0}(f)$

(ii) $J_{1}(e) \cap J_{1}(f) \subset J_{2}(e+f)$

(iii) if $e, f, g$ are orthogonal then $J_{1}(e) \cap J_{1}(f) \subset J_{0}(g)$.

Proof. (i) $J_{2}(e)=P(e) J \subset P\left(J_{0}(f)\right) J \subset J_{0}(f)$. (ii) $P(e+f)=P(e, f)$ on $J_{1}(e) \cap J_{1}(f)$ since $P(e)=0$ on $J_{1}(e)$ and $P(f)=0$ on $J_{1}(f)$, so $P(e+f)^{2}=P(e, f)^{2}=L(e, e) L(f, f)+L(e, f) L(f, e)-L(e, P(e, f) f)$ (by linearized $(0.6))=L(e, e) L(f, f)($ as $L(e, f)=0$ by orthogonality $e \perp f)=$ 
$I$ on $J_{1}(e) \cap J_{1}(f)$, and $J_{1}(e) \cap J_{1}(f) \subset J_{2}(e+f)$. (iii) If $g \perp e, f$ then $g \perp e+f$, so then result follows from (i) and (ii).

Continuing the proof of (2.5), by $(2.9 \mathrm{i})$ an element in $J_{2}\left(\mathscr{E}_{i}\right)$ automatically lies in $J_{0}\left(\mathscr{C}_{j}\right)$ for all $j \neq i$ by orthogonality of $\mathscr{E}_{i}, \mathscr{C}_{j}$ so $J_{i i}=J_{2}\left(\mathscr{E}_{i}\right) \subset \bigcap_{j \neq i} J_{0}\left(\mathscr{E}_{j}\right)$ is the sum

$$
J_{i i}=\sum J_{\left(0, \cdots, 0 ; i_{1}, \cdots, 2, \cdots, i_{m} ; 0, \cdots, 0\right)}
$$

of those Peirce spaces in (2.1) having at least one 2 in the $i$ th range of indices (hence 0's in all other ranges).

By (2.9iii), an element cannot belong to three different $J_{1}\left(\mathscr{E}_{i}\right)$, so $J_{1}(\mathscr{E})=\sum_{i} J_{1}\left(\mathscr{E}_{i}\right) \cap \bigcap_{j \neq i}\left\{J_{1}\left(\mathscr{E}_{j}\right)+J_{0}\left(\mathscr{E}_{j}\right)\right\}$ is the sum

$$
J_{i 0}=\sum J_{\left(0, \cdots, 0 ; i_{1}, \cdots, 1, \cdots, i_{m} ; 0, \cdots, 0\right)} \quad\left(i_{r}=1 \text { or } 0\right)
$$

of those Peirce spaces in (2.1) having no 2's and at least one 1 in the $i$ th range but no 1's (only 0's) in the other ranges, together with the sum

$$
J_{i j}=\sum J_{\left(0, \cdots, 0 ; i_{1}, \cdots, 1, \cdots, i_{m} ; \cdots ; j_{1}, \cdots, 1, \cdots, j_{p} ; 0, \cdots, 0\right)} \quad\left(i_{r}, j_{r}=1 \text { or } 0\right)
$$

of those Peirce spaces in (2.1) having no 2's but at least one 1 in the $i$ th and $j$ th ranges (hence 0 's in all other ranges).

Finally, $J_{0}(\mathscr{E})=\cap J_{0}\left(\mathscr{E}_{i}\right)$ is the Peirce space with no 2 's or 1 's, only 0's:

$$
J_{00}=J_{(0, \cdots, 0 ; \cdots ; 0, \cdots, 0)} \text {. }
$$

This yields the decomposition $J=\oplus J_{i j}$ and the expression for $J_{k}(\mathscr{E})$ and $J_{k}\left(\mathscr{E}_{i}\right)$ in terms of the $J_{i j}$.

Most of the Peirce relations follow directly from (2.2) (P1-3) in the form of the rules

(A) $P\left(J_{i j}\right) J_{k l} \subset \sum\left\{J_{p q} \mid p, q \in\{i, j, k, l, 0\}\right\}$

$$
\left\{J_{i j} J_{k l} J_{m n}\right\} \subset \sum\left\{J_{p q} \mid p, q \in\{i, j, k, l, m, n, 0\}\right\}
$$

(B) $P\left(J_{r s}\right) J$ or $\left\{J_{r s} J J\right\}$ has no component in $J_{t u}$ for $\{t, u\} \cap\{r, s\}=\varnothing$

(C) $\left\{J_{0}\left(\mathscr{E}_{r}\right) J_{0}\left(\mathscr{E}_{r}\right) J_{1}\left(\mathscr{E}_{r}\right)\right\} \subset J_{1}\left(\mathscr{E}_{r}\right)$ has precisely one index $r$

(D) $P\left(J_{1}\left(\mathscr{E}_{r}\right)\right) J_{0}\left(\mathscr{E}_{r}\right) \subset J_{2}\left(\mathscr{E}_{r}\right)+J_{1}\left(\mathscr{E}_{r}\right)$ has at least one index $r$. (We need only verify (A), (B). For (A) note that for all other indices $r$ we have $J_{i j}, J_{k l}, J_{m n}$ and their products falling in $J_{0}\left(\mathscr{E}_{r}\right)=$ $\sum_{p, q \neq r} J_{p q}$. For (B), either $r, s \neq 0$ or $t, u \neq 0$. If $r, s \neq 0$ and $r=s$ then $J_{r s} \subset J_{2}\left(\mathscr{E}_{r}\right)$, while if $r, s, 0 \neq$ then $J_{r s}$ is spanned by the various $J_{1}\left(e_{r p}\right) \cap J_{1}\left(e_{s q}\right) \subset J_{2}\left(e_{r p}+e_{s q}\right)$ by (2.9ii) and orthogonality $\mathscr{E}_{r} \perp \mathscr{E}_{s}$, so in either case $P\left(J_{2}\right) J$ or $\left\{J_{2} J J\right\}$ lies in $J_{2}+J_{1}$ and has no component in $J_{t u} \subset J_{0}$. If $t, u \neq 0$ we similarly have $J_{t u}$ spanned by various $J_{2}$ 's with $J_{r s} \subset J_{0}$, where $P\left(J_{0}\right) J$ and $\left\{J_{0} J J\right\} \subset J_{0}+J_{1}$ have no 
component in $J_{t u} \subset J_{2}$.)

For (2.6), if $\{k, l\} \cap\{i, j\}=\varnothing$ then in particular 0 cannot appear in both pairs. If $i, j \neq 0$ then as above $J_{i j}$ is spanned by $J_{2}$ 's with $J_{k l} \subset J_{0}$, so $P\left(J_{i j}\right) J_{k l}=\left\{J_{i j} J_{k l} J\right\}=0$ follows from $P\left(J_{2}\right) J_{0}=\left\{J_{2} J_{0} J\right\}=0$. Similarly if $k, l \neq 0$ it follows from $P\left(J_{0}\right) J_{2}=\left\{J_{0} J_{2} J\right\}=0$. If $\{k, l\} \not \subset$ $\{i, j, r, s\}$, say $k \neq i, j, r, s$, then the product falls in $P\left(J_{0}\left(\mathscr{E}_{k}\right)\right)\left\{J_{2}\left(\mathscr{E}_{k}\right)+\right.$ $\left.J_{1}\left(\mathscr{E}_{k}\right)\right\}=0$ as long as $k \neq 0$, while if $k=0$ by the above we must have $1 \in\{i, j\} \cap\{r, s\}$ so by symmetry we may take $1=j=r$, therefore the only possible unlinked products are (replacing $s$ by $k$ ) of the form

$$
P\left(J_{i j}\right) J_{0 j} \quad \text { or } \quad\left\{J_{i j} J_{0 j} J_{j k}\right\} \quad(i, j, k \neq 0, k \neq i) \text {. }
$$

Starting from (A) in all instances, we analyze these unlinked products. In the first, either $i=j$, in which case (U1) results from (B) $(r=i)$, or else $i \neq j$, in which case (U2) results from (E) $(r=i)$. In the second product either $i=j \neq k$, whence (U3) results from (C) $(r=k)$, (E) $(r=i)$ and dually if $i \neq j=k$, or else $i, j, k \neq$, whence (U4) results from (C) $(r=i$ and $r=k)$.

To analyze the linked products (P1-11) we again start from (A) in all instances. (P1) results from $P\left(J_{0}\right) J_{0} \subset J_{0}$ and $P\left(J_{2}\right) J_{2} \subset J_{2}+J_{1}$; (P2) results from (D) $(r=j)$ when $j \neq 0$; (P3) results from (B) when $j \neq 0$; (P4) results from (B) $(r=s=i ; r=s=j)$; (P5) results from (B) $(r=s=i)$ plus, when $j \neq 0$, (C) $(r=j)$; (P6) results from (B) $(r=s=i)$; (P7) results from (B) $(r=s=i ; r=j, s=k)$ plus, when $k \neq 0$, (C) $(r=k)$; (P8) results from (C) $(r=i ; r=k)$ when $i, k \neq 0$, and (C) $(r=i)$ when $k=0$; (P9) results (see below) from (C) $(r=k)$ plus (B) $(r=i, s=j)$ when $k \neq 0$, and from (B) $(r=i$, $s=j ; r=i, s=0$ ) when $k=0$; (P10) results (see below) from (D) $(r=i)$ when $i \neq 0$, and from (B) $(r=0, s=j ; r=k, s=0)$ when $i=0$; (P11) results from (C) $(r=i ; r=l)$ when $l \neq 0$, and from (C) $(r=i)$ plus (B) $(r=k, s=0)$ when $l=0$.

To see there are no components of $\left\{J_{i j} J_{i j} J_{i k}\right\}$ in $J_{j k}$ in (P9) ( $i, j \neq 0$ but $k=0$ allowed) we may assume $x_{i j}, y_{i j}, z_{i k}$ lie in Peirce spaces $J_{\left(i_{1}, \cdots, i_{n}\right)}$ of (2.1). Then $y_{i j}$ lies in some $J_{1}\left(e_{j}\right)$ for $e_{j} \in \mathscr{E}_{j}$, whence $x_{i j}$ does too (otherwise it lies in $J_{0}\left(e_{j}\right)$ with $z_{i k}$, and $\{x y z\} \in$ $\left.\left\{J_{0} J_{1} J_{0}\right\}=0\right)$, in 'which case $\{x y z\} \in\left\{J_{1} J_{1} J_{0}\right\} \subset J_{0}\left(e_{j}\right)$. This cannot be true for all $e_{j} \in \mathscr{E}_{j}$ if there is to be a component in $J_{j k}$, so some $e_{j}^{\prime} \in \mathscr{E}_{j}$ has $y_{i j} \in J_{0}\left(e_{j}^{\prime}\right)$, whence $x_{i j} \in J_{1}\left(e_{j}^{\prime}\right)$ (otherwise $x_{i j} \in J_{0}\left(e_{j}^{\prime}\right)$ and again $\left.\{x y z\} \in\left\{J_{0} J_{0} J_{0}\right\} \subset J_{0}\left(e_{j}^{\prime}\right)\right)$. At the same time $x$ lies in some $J_{1}\left(e_{i}\right)$ for $e_{i} \in \mathscr{E}_{i}$, whence $y \in J_{1}\left(e_{i}\right)$ too (if $y \in J_{0}\left(e_{i}\right)$ then $y \in J_{0}\left(e_{i}+e_{j}^{\prime}\right), x \in$ $J_{2}\left(e_{i}+e_{j}^{\prime}\right)$ by $(2.9 \mathrm{ii})$, and $\left.\{x y J\}=0\right)$, whence in turn $z_{i k} \in J_{0}\left(e_{i}\right)$ (otherwise $z \in J_{1}\left(e_{i}\right)$ and $\{x y z\} \in\left\{J_{1} J_{1} J_{1}\right\} \subset J_{1}\left(e_{i}\right)$ would have no component in $\left.J_{j k}\right)$. But then $x, y \in J_{2}\left(e_{i}+e_{j}\right)$ by $(2.9 \mathrm{ii}), z \in J_{0}\left(e_{i}+e_{j}\right)$, 
and again $\{x y z\} \in\left\{J_{2} J_{2} J_{0}\right\}=0$ leads to a contradiction. Thus there never is a component of $\left\{x_{i j} y_{i j} z_{i k}\right\}$ in $J_{j k}$.

To get rid of the components $J_{i j}, J_{i k}$ in (P10) we may assume by symmetry that $j \neq 0$. If $k=0$ then $J_{i k}$ reduces to the term $J_{i 0}$, and if $k \neq 0$ our argument for $J_{i j}$ will apply to $J_{i k}$. Thus we may assume $i, j \neq 0$ and show there is no component in $J_{i j} . J_{i j}$ is spanned by the various $J_{2}\left(e_{i}+e_{j}\right)$ where in (2.9ii) we saw $P\left(e_{i}+e_{j}\right)^{2}=$ $P\left(e_{i}, e_{j}\right)^{2}$, so $J_{i j}$ is spanned by elements $\left\{e_{i} x_{i j} e_{j}\right\}$, where $\left\{\left\{e_{i} x_{i j} e_{j}\right\} y_{j k} z_{k i}\right\}=$ $\left\{e_{i} x_{i j}\left\{e_{j} y_{j k} z_{k i}\right\}\right\}-\left\{\left\{e_{i} x_{i j} z_{k i}\right\} y_{j k} e_{j}\right\}+\left\{z_{k i}\left\{x_{i j} e_{i} y_{j k}\right\} e_{j}\right\}$ (by (0.5)) $=\left\{e_{i} x_{i j}\left\{e_{j} y_{j k} z_{k i}\right\}\right\}$ (by (U), (2.6) for $i, j \neq 0)=\left\{e_{j} y_{j k}\left\{e_{i} x_{i j} z_{k i}\right\}\right\}-\left\{\left\{e_{j} y_{j k} e_{i}\right\} x_{i j} z_{k i}\right\}+\left\{e_{i}\left\{y_{j k} e_{j} x_{i j}\right\} z_{k i}\right\}$ (by $(0.5))=\left\{e_{i}\left\{y_{j k} e_{j} x_{i j}\right\} z_{k i}\right\} \quad$ (by (U), (2.6) again) $\in\left\{J_{0}\left(\mathscr{E}_{j}\right) J J_{0}\left(\mathscr{E}_{j}\right)\right\} \subset$ $J_{0}\left(\mathscr{E}_{j}\right)$ involves no index $j$.

Note that when $\mathscr{E}=\mathscr{E}_{i}$ consists of a single family, the decomposition $J=J_{i i} \oplus J_{i 0} \oplus J_{00}$ and Peirce rules (2.7), (2.8) reduce to (2.2). It is easy to give examples where the unexpected Peirce terms in (2.8) are nonzero if one of the spaces is of the form $J_{i 0}$, since this may include elements which "ought" to belong to $J_{i i}$. For example in (U1), in a matrix algebra $M_{n}(\Phi)$ if we take $\mathscr{E}_{i}=\left\{e_{11}, e_{22}\right\}$ then $e_{11} \in J_{i i}, e_{12} \in J_{i 0}\left(\right.$ not $\left.J_{i i} !\right), e_{22} \in J_{i i}$ so $\left\{e_{11} e_{12} e_{22}\right\}=e_{12} \in J_{i 0} \cap P\left(J_{i i}\right) J_{i 0}$. Similar arguments apply to all components except
(U2)' $\quad P\left(J_{i j}\right) J_{0 j}$ in $J_{i j}$
(P2)' $P\left(J_{i j}\right) J_{i i}$ in $J_{i j}(i=0$ allowed $)$
(P3)' $\quad P\left(J_{i j}\right) J_{i j}$ in $J_{i i}, J_{i 0}$
$(\mathrm{P} 6)^{\prime} \quad\left\{J_{i j} J_{i j} J_{i i}\right\}$ in $J_{i j}$
(P9)' $\left\{J_{i j} J_{i j} J_{i 0}\right\}$ in $J_{i j}$.

It is not clear whether such terms can actually exist.

Whatever their defects and uncertainties, such decompositions are intrinsic.

Proposition 2.10. If $\mathscr{E}=\mathscr{E}_{1} \cup \cdots \cup \mathscr{E}_{n}$ is a union of mutually orthogonal families $\mathscr{E}_{i}$ of compatible tripotents in $J$, then any Jordan triple system $\widetilde{J} \supset J$ inherits the Peirce decomposition

$$
\widetilde{J}=\bigoplus_{i, j \geqq 0} \widetilde{J}_{i j}(\mathscr{E}) \text { for } \widetilde{J}_{i j}(\mathscr{E}) \supset J_{i j}(\mathscr{E}) \text {. }
$$

Any ideal $K \triangleleft J$ inherits the decomposition

$$
K=\oplus K_{i j} \text { for } K_{i j}=K \cap J_{i j}(\mathscr{E}) .
$$

Any bimodule $M$ for $J$ inherits a decomposition

$$
M=\oplus M_{i j} \text { for } M_{i j}=M \cap \widetilde{J}_{i j}(\mathscr{E}) \quad(\widetilde{J}=J \oplus M)
$$

though in general the $M_{i j}$ are not sub J-bimodules. Such supersystems, ideals, or bimodules inherit Peirce multiplication rules 
corresponding to (2.7), (2.8).

Proof. $\mathscr{E}=\cup \mathscr{E}_{i}$ remains compatible in any larger $\widetilde{J}$ since by (1.6) compatibility of $e, f$ is an element condition $\{e e f\}=P(f)^{2}\{e e f\}$ and thus remains true in $\widetilde{J}$. Thus $\mathscr{E}$ decomposes $\widetilde{J}$ with $\widetilde{J}_{i j}=E_{i j}(\widetilde{J}) \supset$ $E_{i j}(J)=J_{i j}$. Since these Peirce projections $E_{i j}=\sum E_{\left(i_{1}, \cdots, i_{n}\right)}$ are multiplication operators, they leave any ideal $K$ invariant, so $K=$ $\sum E_{i j}(K)$ for $E_{i j}(K)=K \cap E_{i j}(J)=K \cap J_{i j}$. In particular, any bimodule $M$ is an ideal in the split null extension $\widetilde{J}=J \oplus M$, so $M=\oplus M_{i j}$.

3. Grid decompositions. We wish to show that semisimple Jordan triple systems are locally unital, indeed have very special sorts of covering families. An orthogonal-collinear family is a family $\mathscr{E}=\left\{e_{i}\right\}$ of tripotents such that any two $e_{i}, e_{j}$ are either orthogonal or collinear; such families are automatically compatible by $(1.8 \mathrm{i}-\mathrm{ii})$.

If $e_{2}$ is collinear with both $e_{1}$ and $e_{3}$ in an orthogonal-collinear family, $e_{1} T e_{2} T e_{3}$, then either $e_{1} T e_{3}$ are collinear (so we have a "line" of tripotents $e_{1}-e_{2}-e_{3}$ ), or else $e_{1} \perp e_{3}$ are orthogonal (so we have the start $e_{1}-e_{2}$ of a quadrangle). This latter configuration is very important-it can always be completed to a true quadrangle

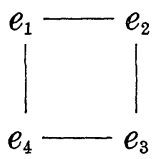

where adjacent corners are collinear and opposite corners are orthogonal.

\section{Quadrangles}

We define a quadrangle of tripotents to be an ordered quadruple $\left\{e_{1}, e_{2}, e_{3}, e_{4}\right\}$ of tripotents such that

$$
\left.e_{i} \top e_{i+1}, e_{i} \perp e_{i+2},\left\{e_{i} e_{i+1} e_{i+2}\right\}=e_{i+3} \quad \text { (indices } \bmod 4\right) \text {. }
$$

Examples are $\left\{E_{i r}, E_{i s}, E_{j s}, E_{j r}\right\}$ in $M_{p, q}(D)(p, q \geqq 2), \quad\left\{F_{i j}, F_{i l}, F_{k l}\right.$, $\left.F_{k j}\right\}$ in $S_{n}(C)(n \geqq 4)$, and $\left\{H_{i j}, H_{i l}, H_{k l}, H_{k j}\right\}$ in $H_{n}\left(D, D_{0}, j\right)(n \geqq 4)$.

QUADRANGLE LEMMA 3.2. $e_{1} T e_{2} T e_{3}, e_{1} \perp e_{3}$ suffices for quadrangularity: this implies $e_{4}=\left\{e_{1} e_{2} e_{3}\right\}$ is a tripotent collinear with $e_{1}, e_{3}$ and orthogonal to $e_{2}$, such that $\left\{e_{2} e_{3} e_{4}\right\}=e_{1},\left\{e_{3} e_{4} e_{1}\right\}=e_{2},\left\{e_{4} e_{1} e_{2}\right\}=$ $e_{3}$, so $\left\{e_{1}, e_{2}, e_{3}, e_{4}\right\}$ is a quadrangle. 
Proof. One can verify this directly, or use the exchange automorphisms of [7] taking $e_{1}, e_{2}, e_{3}, e_{4} \rightarrow e_{2}, e_{1},-e_{4},-e_{3}$ and $e_{1}, e_{2}, e_{3}$, $e_{4} \rightarrow-e_{4}, e_{3}, e_{2},-e_{1}$.

The Peirce decompositions (2.1) and (2.2) simplify in the case of a quadrangle

3.3. Quadrangular Decomposition. If $\mathscr{E}=\left\{e_{1}, e_{2}, e_{3}, e_{4}\right\}$ is a quadrangle of tripotents in $J$ then the multiplication operators satisfy
(i ) $L\left(e_{i}, e_{i+1}\right)=L\left(e_{i+3}, e_{i+2}\right) \quad$ (indices modulo 4$)$
(ii) $P\left(e_{i}\right) P\left(e_{i+1}\right)=P\left(e_{i+3}\right) P\left(e_{i+2}\right)$
(iii) $L\left(e_{1}, e_{1}\right)-L\left(e_{2}, e_{2}\right)+L\left(e_{3}, e_{3}\right)-L\left(e_{4}, e_{4}\right)=0$.

The Peirce decomposition relative to $\mathscr{E}$ is $J=J_{2}(\mathscr{E}) \oplus J_{1}(\mathscr{E}) \oplus J_{0}(\mathscr{E})$ where

$$
\begin{aligned}
& J_{2}(\mathscr{S})=\left\{J_{(2200)}+J_{(0022)}+J_{(2002)}+J_{(0220)}\right\}+\left\{J_{(2101)}+J_{(1012)}\right. \\
& \left.\quad+J_{(1210)}+J_{(0121)}\right\} \\
& J_{1}(\mathscr{E})=\left\{J_{(1111)}+J_{(1001)}+J_{(0110)}\right\}+\left\{J_{(1100)}+J_{(0011)}\right\} \\
& J_{0}(\mathscr{E})=J_{(0000)}
\end{aligned}
$$

while all other Peirce spaces vanish.

Proof. For (i) we have $L\left(e_{i}, e_{i+1}\right)=L\left(e_{i},\left\{e_{i+2} e_{i+3} e_{i}\right\}\right)=-L\left(e_{i+3}\right.$, $\left.\left\{e_{i+2} e_{i} e_{i}\right\}\right)+L\left(\left\{e_{i} e_{i+2} e_{i+3}\right\}, e_{i}\right)+L\left(\left\{e_{i} e_{i} e_{i+3}\right\}, e_{i+2}\right)$ (by linearized $\left.(0.3)\right)=-$ $0+0+L\left(e_{i+3}, e_{i+2}\right)$ by quadrangularity. For (iii) we have $L\left(e_{2}, e_{2}\right)+$ $L\left(e_{4}, e_{4}\right)=L\left(e_{2},\left\{e_{3} e_{4} e_{1}\right\}\right)+L\left(e_{4},\left\{e_{3} e_{2} e_{1}\right\}\right)=L\left(\left\{e_{2} e_{3} e_{4}\right\}, e_{1}\right)+L\left(\left\{e_{4} e_{1} e_{2}\right\}, e_{3}\right)$ (by linearized $(0.3))=L\left(e_{1}, e_{1}\right)+L\left(e_{3}, e_{3}\right)$. For (ii) $P\left(e_{i+3}\right) P\left(e_{i+2}\right)=$ $P\left(\left\{e_{i} e_{i+1} e_{i+2}\right\}\right) P\left(e_{i+2}\right)=\left\{P\left(e_{i}\right) P\left(e_{i+1}\right) P\left(e_{i+2}\right)+P\left(e_{i+2}\right) P\left(e_{i+1}\right) P\left(e_{i}\right)+L\left(e_{i}\right.\right.$, $\left.\left.e_{i+1}\right) P\left(e_{i+2}\right) L\left(e_{i+2}, e_{i+3}\right)-P\left(e_{i+2}, P\left(e_{i}\right) P\left(e_{i+1}\right) e_{i+2}\right)\right\} P\left(e_{i+2}\right)$ (by (0.8) and (i) above $)=P\left(e_{i}\right) P\left(e_{i+1}\right) P\left(e_{i+2}\right)^{2}$ (by $e_{i+2} \perp e_{i}, P\left(e_{i+1}\right) e_{i+2}=0$, and (0.2) with $\left.P\left(e_{i+2}\right) e_{i+3}=0\right)=P\left(e_{i}\right)\left\{P\left(\left\{e_{i+1} e_{i+2} e_{i+2}\right\}\right)+P\left(e_{i+1}, P\left(e_{i+2}\right)^{2} e_{i+1}\right)-P\left(e_{i+2}\right)^{2} P\left(e_{i+1}\right)-\right.$ $\left.L\left(e_{i+2} e_{i+2}\right) P\left(e_{i+1}\right) L\left(e_{i+2}, e_{i+2}\right)\right\} \quad($ by $\quad(0.8))=P\left(e_{i}\right) P\left(e_{i+1}\right) \quad\left(\right.$ by $\quad e_{i} \perp e_{i+2}$, $\left.P\left(e_{i+2}\right) e_{i+1}=0, P\left(e_{i}\right)\left\{e_{i+2} e_{i+2} J\right\} \subset P\left(e_{i}\right)\left\{J_{0}\left(e_{i}\right) J J\right\}=0\right)$.

To obtain (iv) we show the 81 terms $J_{I}=J_{\left(i_{1}, i_{2}, i_{3}, i_{4}\right)}$ for $I \in$ $\{2,1,0\}^{4}$ of the Peirce decomposition (2.1) relative to $\mathscr{E}=\left\{e_{1}, e_{2}, e_{3}, e_{4}\right\}$ reduce to the above 14 terms, i.e., the other 67 vanish. From $e_{1} \perp$ $e_{3}, e_{2} \perp e_{4}$ and (2.9i) we see that whenever $I$ has an index $i_{k}=2$ then $J_{I}$ vanishes unless $i_{k+2}=0$. This gets rid of 45 spaces

$$
J_{(2 i 2 j)}=J_{(2 i 1 j)}=J_{(1 i 2 j)}=J_{(i 2 j 2)}=J_{(i 2 j 1)}=J_{(i 1 j 2)}=0 \text {. }
$$

Applying (iii) to $J_{\left(i_{1}, i_{2}, i_{j}, i_{4}\right)}$ shows $\left(i_{1}-i_{2}+i_{3}-i_{4}\right) I=0$ there, so $J_{I}=0$ unless $i_{1}-i_{2}+i_{3}-i_{4}=0$ on $J_{I}$. This gets rid of 16 more spaces 


$$
\begin{aligned}
& J_{(2001)}=J_{(2100)}=J_{(0210)}=J_{(1200)}=J_{(1002)}=J_{(0021)}=J_{(0120)}=J_{(0012)}=0 \\
& J_{(1101)}=J_{(1110)}=J_{(1011)}=J_{(011)}=J_{(1000)}=J_{(0100)}=J_{(0010)}=J_{(0001)}=0 .
\end{aligned}
$$

If we assume $1 / 2 \in \Phi$ we could deduce the remaining 6 spaces vanish,

$$
J_{(2000)}=J_{(0200)}=J_{(0020)}=J_{(0101)}=J_{(1010)}=0,
$$

but in general we must give a different argument for these: they vanish because of $J_{0}\left(e_{i}\right) \cap J_{0}\left(e_{i+2}\right) \subset J_{0}\left(e_{i+1}\right) \cap J_{0}\left(e_{i+3}\right)$, which follows since for $e_{i+1}$ we have $L\left(e_{i+1}, e_{i+1}\right) x=\left\{e_{i+1}\left\{e_{i+2} e_{i+3} e_{i}\right\} x\right\}=-\left\{e_{i+1}\left\{e_{i+2} x e_{i}\right\} e_{i+3}\right\}+$ $\left\{e_{i+1} e_{i+2}\left\{e_{i+3} e_{i} x\right\}\right\}+\left\{e_{i+1} e_{i}\left\{e_{i+3} e_{i+2} x\right\}\right\}=0$ by linearized (0.3) and $x \perp e_{i}, e_{i+2}$, and also $P\left(e_{i+1}\right) x=P\left(\left\{e_{i+2} e_{i+3} e_{i}\right\}\right) x=\left\{P\left(e_{i+2}\right) P\left(e_{i+3}\right) P\left(e_{i}\right)+P\left(e_{i}\right) P\left(e_{i+3}\right) P\left(e_{i+2}\right)+\right.$ $\left.P\left(e_{i}, e_{i+2}\right) P\left(e_{i+3}\right) P\left(e_{i}, e_{i+2}\right)-P\left(P\left(e_{i+2}\right) e_{i+3}, P\left(e_{i}\right) e_{i+3}\right)\right\} x=0$ using linearized (0.1) plus $x \perp e_{i}, e_{i+2}$ plus $e_{i} \perp e_{i+3}$. Thus the 67 spaces vanish, leaving the 14 spaces of (iv).

Another way to see the last 6 spaces vanish is to note that for collinear tripotents, certain of the Peirce spaces are tied to each other.

LEMma 3.4. The Peirce spaces relative to collinear tripotents $e T f$ satisfy

(i) $\quad J_{(20)}=P(e) J_{(22)}, J_{(02)}=P(f) J_{(22)}, J_{(22)}=P(e) J_{(20)}=P(f) J_{(02)}$

(ii) $J_{(10)}=L(e, f) J_{(01)}, J_{(01)}=L(f, e) J_{(10)}$.

Proof. (i) $P(e)^{2}=I$ on $J_{2}(e)$ shows via (1.2) that $J_{(20)}=P(e) P(e) J_{(20)} \subset$ $P(e) J_{(22)} \subset J_{(20)}$, so $J_{(20)}=P(e) J_{(22)}, J_{(22)}=P(e)^{2} J_{(22)}=P(e) J_{(20)}$, and dually for $f$.

(ii) $J_{(10)}=\left\{e e J_{10}\right\}=\left\{(L(e, f) f) e J_{10}\right\} \subset L(e, f)\left\{f e J_{(10)}\right\}-\{f e(L(e$, $\left.\left.f) J_{(10)}\right)\right\}+\left\{f(L(f, e) e) J_{(10)}\right\}$ (by $\left.(0.5)\right) \subset L(e, f) J_{(10)} \subset J_{(01)}$ (since $\left\{J f J_{(10)}\right\} \subset$ $\left.\left\{J f J_{0}(f)\right\}=0\right)$, so $J_{(10)}=L(e, f) J_{(01)}$, and dually.

These are special instances of a global exchange automorphism [7] which exchanges $e$ and $f$. From these we see $J_{(2000)}=P\left(e_{1}\right) J_{(2202)}=$ $0, J_{(1010)} \subset L\left(e_{1}, e_{2}\right) J_{(01)} \cap J_{0}\left(e_{4}\right) \subset\left\{J_{1}\left(e_{4}\right) J_{0}\left(e_{4}\right) J\right\} \cap J_{0}\left(e_{4}\right)=0$, and similarly for the other spaces.

\section{Rigidity}

Two orthogonal tripotents $e \perp f$ are automatically rigid in the sense that not merely $e$, but the whole Peirce space $J_{2}(e)$ governed by $e$, falls in $J_{0}(f)$ by (2.9i). Unfortunately the analogous property need not hold for collinear tripotents. We say collinear $e, f$ are rigid or rigidly imbedded in $J$ if the whole Peirce space governed by $e$ falls in $J_{1}(f)$ 


$$
J_{2}(e) \subset J_{1}(f) \quad(e \top f \text { rigid }) .
$$

In view of the Peirce decomposition $J_{2}(e)=J_{(22)} \oplus J_{(21)} \oplus J_{(20)}$ in (1.2), rigidity means $J_{2}(e)=J_{(21)}$, i.e., $J_{(22)}=J_{(20)}=0$. From (3.4i) it suffices if either $J_{(22)}$ or $J_{(20)}$ vanishes, since they are interchanged by $P(e)$. In particular

$$
e T f \text { are rigid iff } J_{(22)}=0,
$$

which shows rigidity is symmetric. The condition $J_{(22)}=0$ is that $e$ and $f$ do not "overlap" in $J$, in the sense that they have no common elements in their 2-spaces.

An important situation where rigidity is automatic is the case of division tripotents, those $e$ for which $J_{2}(e)$ is a division system all of whose nonzero elements $x$ are invertible in the sense that $P(x)$ is an invertible operator. Such tripotents are found in abundance in systems satisfying the d.c.c. on inner ideals. Slightly more general are the domain tripotents, for which $J_{2}(e)$ is a domain in the sense that all nonzero $x$ are cancellable, i.e., $P(x)$ is injective; this is equivalent to the condition that there be no zero divisors $P(x) y=0$ for $x, y \neq 0$. At the other extreme from this case where $J_{2}(e)$ is small is that where $J_{2}(e)$ is large, namely the case of a full (maximal) tripotent $e$ with $J_{0}(e)=0$.

Proposition 3.7. If $e$ is a full or domain tripotent, then any tripotent $f$ collinear with $e$ is automatically rigidly imbedded with $e$,

$$
J_{2}(e) \subset J_{1}(f) \text {. }
$$

If $e$ is a domain tripotent then a nonzero tripotent $f$ in $J_{1}(e)$ will be collinear with $e$ as soon as $P(f) e=0$.

Proof. Suppose $e$ and $f$ are collinear. If $e$ is full then $J_{0}(e)=0$ implies $J_{(02)}=0$ and $e, f$ are rigid. Suppose now that $e$ is a domain tripotent, $J_{2}(e)$ is a domain. But $P\left(J_{(22)}\right) e \subset P\left(J_{2}(f)\right) J_{1}(f)=0$ by (1.2), so $J_{(22)}=0$ and hence $e, f$ are rigid.

Now suppose $e$ is a domain tripotent and $f$ a tripotent in $J_{1}(e)$ with $P(f) e=0$. Then collinearity reduces to $\{f f e\}=e$. Writing $e=x_{2}+x_{1}+x_{0}$ for Peirce elements $x_{i} \in J_{i}(f)$, we have $x_{2}=P(f)^{2} e=0$ by hypothesis, so $x_{1}=\{f f e\} \in\left\{J_{1}(e) J_{1}(e) J_{2}(e)\right\} \subset J_{2}(e)$, so also $x_{0}=e-$ $x_{1} \in J_{2}(e)$, yet $P\left(x_{0}\right) x_{1}=0$ by (1.2). Since $J_{2}(e)$ is a domain this forces one of $x_{1}, x_{0}$ to vanish. Here $x_{1}=0$ would imply $e=x_{0}$ is orthogonal to $f$, so instead it must be $x_{0}$ that vanishes, and $e=x_{1}=\{f f e\}$.

Rigidity is not an intrinsic property of the tripotents, it depends very much on the imbedding. For example, $e=E_{11}$ and $f=E_{12}$ are 
rigidly imbedded in $M_{1,2}(D)$, but not in the larger system $H_{3}\left(D, D_{0}\right.$, j) (imbedded via $e=H_{12}, f=H_{13}$ ), since here $e$ and $f$ overlap on $J_{(22)}=D_{0}[11]$.

\section{Grids}

An orthogonal-collinear family $\mathscr{E}=\left\{e_{i}\right\}$ is rigidly imbedded or rigid in $J$ if each collinear pair $e_{i}, e_{j}$ is rigid (we observed that orthogonal pairs are always rigid). Thus for each $i, j$ either $J_{2}\left(e_{i}\right) \subset$ $J_{0}\left(e_{j}\right)$ or $J_{2}\left(e_{i}\right) \subset J_{1}\left(e_{j}\right)$, according as $e_{i} \perp e_{j}$ or $e_{i} \top e_{j}$.

A grid is a rigid orthogonal-collinear family $\mathscr{C}$ which covers $J$ and is closed under multiplication, in the sense that for distinct $e, f, g \in \mathscr{E}\{e f g\}$ is zero or (up to sign) a tripotent in $\mathscr{E}$. (By orthogonal-collinearity, $P(e) f$ and $\{e e f\}$ automatically vanish or fall in $\mathscr{E}$.) $J$ is basically determined by $\mathscr{E}$.

GRID Decomposition 3.8. If the Jordan triple system $J$ possesses a grid $\mathscr{E}$ then it has a grid decomposition

$$
J=\bigoplus_{e \in \mathscr{Y}} J_{e}
$$

for

$$
J_{e}=J_{2}(e)=J_{2}(e) \cap\left\{\bigcap_{f \uparrow e} J_{1}(f)\right\} \cap\left\{\bigcap_{g_{\perp e}} J_{0}(g)\right\} .
$$

These subspaces multiply according to

(G1) $P\left(J_{e}\right) J_{e} \subset J_{e}, P\left(J_{e}\right) J_{f}=0$

(G2) $\left\{J_{e} J_{e} J_{f}\right\} \subset J_{f}$ if $e T f$ and $\left\{J_{e} J_{e} J_{f}\right\}=0$ if $e \perp f$

(G3) $\left\{J_{e} J_{f} J_{g}\right\}=0$ if $e \perp f$ or $f \perp g$ or $e T g$ (i.e., unless $e T f T$

$$
g \perp e)
$$

(G4) $\left\{J_{e} J_{f} J_{g}\right\} \subset J_{h}$ for $e T f T g \perp e$, where $\{e, f, g, h\}$ forms a quadrangle with $h=\{e f g\}$.

$J$ decomposes into a direct sum $J=J_{1} \boxplus \cdots \boxplus J_{m}$ of ideals $J_{i}=$ $J_{2}\left(\mathscr{E}_{i}\right)=\sum_{e \in \mathscr{S}_{i}} J_{2}(e)$ corresponding to the connected components $\mathscr{E}_{i}$ of $\mathscr{E}$ under the equivalence relation generated by collinearity $(e \sim f$ iff $e T e_{1} \top \cdots T e_{n} T f$ for some $e_{i}$ ).

Proof. The description of $J_{2}(e)$ follows from orthogonal-collinearity and rigidity. Thus the spaces $J_{e}$ are independent, and by the covering property $J=J_{2}(\mathscr{E})$ they span $J$, so $J=\oplus J_{e}$. The relations (G1), (G2) hold whenever $e, f$ are rigidly imbedded, by the Peirce relations (1.2). For (G3) note that if $e T f T g$ the product vanishes when $e T g$ by rigid collinearity, $\left\{J_{e} J_{f} J_{g}\right\} \subset J_{2}(e) \cap J_{0}(f) \cap$ $J_{2}(g)=0$ by (3.6). For (G4), recall by (3.2) that $\{e, f, g, h\}$ does form a quadrangle whenever $e T f T g \perp e$. By rigidity, $\left\{J_{e} J_{f} J_{g}\right\} \subset\left\{J_{1}(h) J_{0}(h) J_{1}(h)\right\} \subset$ $J_{2}(h)=J_{h}$, where we have used the closure property of a grid to 
insure that $\pm h$ belongs to $\mathscr{E}$ (note $J_{-h}=J_{h}$ ).

From these rules it is clear that the $J_{2}\left(\mathscr{E}_{i}\right)$ are orthogonal ideals summing to $J$ (if $e_{i} \in \mathscr{E}_{i}, e_{j} \in \mathscr{E}_{j}$ distinct then $e_{i} \perp e_{j}$, and a product is always connected to both outer factors).

This allows us to concentrate on "connected" grids. To a connected grid we can attach a coordinate algebra $D$ : we choose a tripotent $e \in \mathscr{E}$ and introduce $D=J_{2}(e)$. By connectivity all $J_{2}(f)$ are isomorphic to $J_{2}(e)$ by a chain of exchange automorphisms $T_{e_{i}, e_{i}+1}$ [7], so $J$ is a direct sum of copies of $D$. The exact description of $J$ reduces to the selection of canonical identification or symmetry maps $J_{2}(f) \rightarrow J_{2}(e)$, and the description of the collinear product (G2) and quadrangular products (G4). We will carry out this program for rectangular, symplectic, and hermitian grids in a subsequent paper [7].

\section{Examples}

We now want to exhibit grids for all semisimple triple systems.

EXAMPLE 3.9 (Unital grid). If $J$ is a unital Jordan algebra, then $J$ has as covering grid $\mathscr{E}=\{1\}$.

Example $3.10\left(1 \times 2\right.$ Grid). The triple system $M_{1,2}(D)$ of $1 \times 2$ matrices (as in (0.9)) over an alternative algebra with involution has covering grid $\mathscr{E}=\left\{E_{11}, E_{12}\right\}$ consisting of two rigidly collinear tripotents $E_{11}, E_{12}$. Here $J_{2}\left(E_{1 j}\right)=D E_{1 j}, J_{1}\left(E_{1 j}\right)=D E_{1 k}, J_{0}\left(E_{1 j}\right)=0$, $(k=3-j)$.

EXAmple 3.11 (Rectangular grid). The triple system $M_{p, q}(D)$ of rectangular matrices (as in (0.9)) has as covering grid the rectangular grid $\mathscr{E}=\left\{E_{i j}\right\}$ of all rectangular matrix units $E_{i j}$. Here $J_{2}\left(E_{i j}\right)=$ $D E_{i j}, J_{1}\left(E_{i j}\right)=\sum_{l \neq j} D E_{i l}+\sum_{k \neq i} D E_{k j}, J_{0}\left(E_{i j}\right)=\sum_{k \neq i, l \neq j} D E_{k l}$, so $E_{i j}$, $E_{k l}$ are rigidly collinear if they share a common row index $i=k$ or column index $j=l$, and are orthogonal otherwise.

EXAMPLE 3.12 (Symplectic grid). The symplectic triple system $S_{n}(C)$ of alternating matrices (as in $(0.10)$ ) has symplectic grid $\mathscr{E}=$ $\left\{F_{i j} \mid i<j\right\}$ consisting of the symplectic matrix units $F_{i j}\left(F_{j i}=-F_{i j}\right.$, $\left.F_{i i}=0\right)$. Here $J_{2}\left(F_{i j}\right)=C F_{i j}, J_{1}\left(F_{i j}\right)=\sum_{k \neq i, j} C F_{i k}+C F_{k j}, J_{0}\left(F_{i j}\right)=$ $\sum_{k, l, i, j \neq} C F_{k l}$ so $F_{i j}, F_{k l}$ are rigidly collinear if they share a common index and are orthogonal otherwise.

Example 3.13 (Hermitian grid). The triple system $H_{n}\left(D, D_{0}, J\right)$ of $n \times n$ hermitian matrices (as in (0.12)) is an isotope of a Jordan 
algebra, hence trivially has unital covering grid $\mathscr{E}=\{1\}$. It also has an orthogonal-collinear cover $\mathscr{E}=\left\{H_{i j} \mid i<j\right\}$, of all off-diagonal hermitian matrix units $H_{i j}=H_{j i}(i<j)$; here $J_{2}\left(H_{i j}\right)=D_{0}[i i]+$ $D[i j]+D_{0}[j j], J_{1}\left(H_{i j}\right)=\sum_{k \neq i, j} D[i k]+D[k j], J_{0}\left(H_{i j}\right)=\sum_{k \neq i, j} D_{0}[k k]+$ $\sum_{k, l, i, j \neq} D[k l]$, so $H_{i k}, H_{k l}$ are collinear if they share a common index and are orthogonal otherwise. However this compatible family is not a grid in the sense of (3.8) since the collinear tripotents are not rigidly collinear: $J_{2}\left(H_{i j}\right) \cap J_{2}\left(H_{i k}\right)=D_{0}[i i] \neq 0$. The hermitian grid $\mathscr{E}=\left\{H_{i j} \mid i \leqq j\right\}$ IS NOT A GRID; it remains compatible, though no longer orthogonal-collinear.

EXAMPLE 3.14. If $J=\Phi^{n}$ with $P(x) y=2\langle x, y\rangle x-\langle x, S x\rangle S y$ for $\langle x, y\rangle=\sum x_{i} y_{i}$ the standard inner product on $\Phi^{n}$ and $S$ the reflection in some subspace of $\Phi^{n}$, then $J$ contains invertible tripotents $e$ where $\langle e, e\rangle=1$ and $S e= \pm e$, hence $J$ is an isotope of a Jordan algebra and has covering grid $\mathscr{E}=\{e\}$.

The remaining basic examples of Jordan triples are really Jordan pairs. For our present purposes we prefer to consider Jordan pairs as polarized triple systems, consisting of a Jordan triple system $J$ together with a decomposition $J=J_{+} \oplus J_{-}$such that $P\left(J_{\varepsilon}\right) J_{\varepsilon}=$ $\left\{J_{\varepsilon} J_{\varepsilon} J\right\}=0 \quad(\varepsilon= \pm 1)$. Thus the only nontrivial products have the form $P\left(J_{\varepsilon}\right) J_{-\varepsilon}$ or $\left\{J_{\varepsilon} J_{-\varepsilon} J_{\varepsilon}\right\}$ falling in $J_{\varepsilon}$. Then $J=\left(J_{+}, J_{-}\right)$consists of a pair of spaces acting on each other like Jordan triple systems, but with trivial action on themselves.

The polarized triples we need to consider have the special form $\widetilde{J}=J \oplus J$ obtained by pairing two copies of the same Jordan triple system $J_{+}=J_{-}=J$ with $P\left(x_{\varepsilon}\right) y_{-\varepsilon}=P(x) y . \quad \widetilde{J}$ is isomorphic to $J \otimes \Omega$ for $\Omega=\Phi_{+} \boxplus \Phi_{-}$, with $\widetilde{P}(x) y=P(x) y^{*}$ where the exchange involution $(x \oplus y)^{*}=y \oplus x$ is induced from the exchange involution on $\Omega$. The map $x \rightarrow \widetilde{x}=x \oplus x$ is an isomorphism of $J$ with $H(\widetilde{J}, *)$. If $\mathscr{E}=\left\{e_{i}\right\}$ is a compatible or orthogonal-collinear cover or grid for $J$, then $\tilde{\mathscr{E}}=\left\{\widetilde{e}_{i}\right\}$ is a family of the same sort which covers $\widetilde{J}$ since $\widetilde{J}_{k}\left(\widetilde{e}_{i}\right)=J_{k}\left(e_{i}\right) \oplus J_{k}\left(e_{i}\right)$.

EXAMPLE 3.15. If $\widetilde{J}=J \oplus J$ results from doubling a Jordan triple system $J$ having grid $\mathscr{E}$, then the Jordan pair or polarized Jordan triple system $\widetilde{J}$ has grid $\tilde{\mathscr{E}}$.

Since all semisimple Jordan pairs with d.c.c. on all inner ideals are direct sums of simple systems of the above types 3.9-3.15 ([2, p. 138-139]), as are the semisimple Jordan triple systems finitedimensional over an algebraically closed field of characteristic $\neq 2$ ([3, Th. 10.3, p. 63]), and grids are inherited by direct sums, we 
have

GRID THEOREM 3.16. Any semisimple Jordan pair with d.c.c. on inner ideals, or semisimple Jordan triple system finite-dimensional over an algebraically closed field of characteristic $\neq 2$, has a covering grid of tripotents which are pairwise orthogonal or collinear.

It will be important when we try to lift tripotents, that the covers are not merely compatible, but actually orthogonal-collinear.

4. Peirce reflections. A Peirce decomposition $J=J_{2} \oplus J_{1} \oplus J_{0}$ relative to a tripotent $e$ determines an important automorphism of period 2, the Peirce reflection $S_{e}\left(x_{2}+x_{1}+x_{0}\right)=x_{2}-x_{1}+x_{0}$,

$$
S_{e}=E_{2}-E_{1}+E_{0}=B(e, 2 e) \text { with } S_{e}=(-1)^{i} \text { on } J_{i}(e) .
$$

These generate a normal subgroup of the group of automorphism, $T S_{e} T^{-1}=S_{T e}$, and play an important role in many applications.

We wish to try the same thing for an arbitrary compatible family of tripotents $\mathscr{E}$ in place of $e$. The Peirce reflection $S_{\mathscr{E}}$ relative to this family is defined to be

$$
S_{\mathscr{E}}=E_{2}(\mathscr{E})-E_{1}(\mathscr{E})+E_{0}(\mathscr{E}) \text {, so } s_{\mathscr{E}}=(-1)^{i} \text { on } J_{i}(\mathscr{E})
$$

for the Peirce projections $E_{i}(\mathscr{E})$ of $J$ on $J_{i}(\mathscr{E})$ in (2.2). These are normalized by automorphisms,

$$
T S \mathscr{E} T^{-1}=S_{T(\mathscr{E})}
$$

However, in general the invertible linear operator $S_{\mathscr{\varepsilon}}$ of period 2 is not expressible as a $B$ operator and is not an automorphism of the triple system. The conditions for it to be an automorphism are

Lemma 4.3. The Peirce reflection $S_{\mathscr{E}}$ relative to a compatible family $\mathscr{E}=\left\{e_{1}, \cdots, e_{n}\right\}$ of tripotents is an automorphism of $J$ if the Peirce decomposition $J=J_{2} \oplus J_{1} \oplus J_{0}\left(\right.$ for $J_{i}=J_{i}(\mathscr{E})$ ) satisfies the Peirce rules

$$
\begin{array}{ll}
\text { (i) } & P\left(J_{1}\right) J_{1} \subset J_{1}, P\left(J_{2}\right) J_{2} \subset J_{2} \\
\text { (ii) } & P\left(J_{1}\right)\left(J_{2}+J_{0}\right) \subset J_{2}+J_{0}, P\left(J_{2}\right) J_{1} \subset J_{1} \\
\text { (iii) } & \left\{J_{1} J_{1}\left(J_{2}+J_{0}\right)\right\} \subset J_{2}+J_{0} \\
\text { (iv) } & \left\{J_{2} J_{2} J_{1}\right\} \subset J_{1} .
\end{array}
$$

Proof. The map $S=I \oplus-I$ on $J_{+} \oplus J_{-}$is an automorphism if the subspaces $J_{\varepsilon}\left(\varepsilon= \pm\right.$ ) satisfy $(*) P\left(J_{\varepsilon}\right) J_{\varepsilon} \subset J_{\varepsilon}$, (**) $P\left(J_{\varepsilon}\right) J_{-\varepsilon} \subset J_{-\varepsilon}$, (***) $L\left(J_{\varepsilon}, J_{\varepsilon}\right) J_{-\varepsilon} \subset J_{-\varepsilon}$. By (4.2), for $J_{+}=J_{2}+J_{0}$ and $J_{-}=J_{1}$ these 
reduce to $(*)=(\mathrm{i}),(* *)=(\mathrm{ii})$, and $(* * *)=(\mathrm{iii})+(\mathrm{iv})$.

These conditions are necessary if $J$ has no 2-torsion. On the other hand, if $J$ has characteristic 2 then all Peirce reflections reduce to the identity map, which is automatically an automorphism.

We verify these conditions for two special situations which are important in constructing symmetries of matrix systems [7].

Proposition 4.4. The Peirce reflection $S_{\mathscr{B}}$ will be an automorphism in either of the two following cases: if $\mathscr{E}=\{e, f\}$ for two collinear tripotents $e, f$ with Peirce decomposition

$$
J_{2}(\mathscr{E})=J_{(21)} \oplus J_{(12)}, J_{1}(\mathscr{E})=J_{(11)} \oplus J_{(10)} \oplus J_{(01)}, J_{0}(\mathscr{E})=J_{(00)}
$$

satisfying the conditions

(2a) $J_{(22)}=J_{(20)}=J_{(02)}=0$

(2b) $P\left(J_{(11)}\right) e=P\left(J_{(11)}\right) f=0$

(2c) $L\left(J_{(01)}, J_{(11)}\right) e=L\left(J_{(10)}, J_{(11)}\right) f=0$

or if $\mathscr{E}=\{e, f, k\}$ for three pairwise collinear tripotents $e, f, k$ with Peirce decomposition

$$
\begin{gathered}
J_{2}(\mathscr{E})=J_{(220)} \oplus J_{(202)} \oplus J_{(022)} \oplus J_{(211)} \oplus J_{(121)} \oplus J_{(12),}, J_{0}(\mathscr{E})=J_{(000)}, \\
J_{1}(\mathscr{E})=J_{(110)} \oplus J_{(101)} \oplus J_{(011)}
\end{gathered}
$$

satisfying

$$
\begin{aligned}
\text { (3a) } J_{(2 i j)}= & J_{(j 2 i)}=J_{(i j 2)}=0 \text { unless } i=j=1 \text { or } i=2, j=0 \text {, or } \\
& i=0, j=2 \\
\text { (3b) } \quad J_{(111)}= & J_{(100)}=J_{(010)}=J_{(001)}=0 .
\end{aligned}
$$

Proof. Consider first the case $\mathscr{E}=\{e, f, k\}$ of three tripotents. To verify the conditions (i)-(iv) of (4.3,) by symmetry in $e, f, k$ and the fact that always $L\left(J_{I}, J_{I}\right) J_{K} \subset J_{K}$, it suffices to verify (i'-ii') $P\left(J_{(110)}\right)$ and $P\left(J_{(110)}, J_{(101)}\right)$ and $P\left(J_{(220)}\right)$ and $P\left(J_{(220)}, J_{(022)}+J_{(112)}+J_{(211)}\right)$ and $P\left(J_{(211)}\right)$ and $P\left(J_{(211)}, J_{(122)}\right)$ leave $J_{1}$ and $J_{2}+J_{0}$ invariant, (iii') $L\left(J_{(110)}, J_{(101)}\right)$ leaves $J_{2}+J_{0}$ invariant, (iv') $L\left(J_{(211)}, J_{(121)}\right)$ and $L\left(J_{(211)}, J_{(220)}\right)$ and $L\left(J_{(220)}, J_{(211)}\right)$ leave $J_{1}$ invariant (noting that $L\left(J_{(211)}, J_{(022)}\right)=0$ etc. when corresponding adjacent indices are 2,0). By the individual Peirce relations, the only nontrivial products in (i'-ii') are $P\left(J_{(110)}\right)\left(J_{(110)}+\right.$ $\left.J_{(220)}+J_{(000)}\right) \subset J_{(110)}+J_{(000)}+J_{(220)}, P\left(J_{(110)}, J_{(101)}\right)\left(\left\{J_{(110)}+J_{(101)}+J_{(011)}\right\}+\right.$ $\left.\left\{J_{(211)}+J_{(000)}\right\}\right) \subset\left\{J_{(101)}+J_{(110)}+J_{(200)}\right\}+\left\{J_{(000)}+J_{(211)}\right\}$ where $J_{(200)}=0$ by (3a), $\quad P\left(J_{(220)}\right) J_{(220)} \subset J_{(220)}, P\left(J_{(220)}, J_{(022)}\right) J_{(121)} \subset J_{(121)}, P\left(J_{(220)}, J_{(112)}\right)\left(J_{(211)}+\right.$ $\left.J_{(121)}\right) \subset J_{(121)}+J_{(211)}, P\left(J_{(220)}, J_{(211)}\right)\left(J_{(220)}+J_{(211)}\right) \subset J_{(211)}+J_{(220)}, P\left(J_{(211)}\right)\left(J_{(211)}+\right.$ $\left.J_{(220)}+J_{(202)}\right) \subset J_{(211)}+J_{(202)}+J_{(220)}, P\left(J_{(211)}, J_{(112)}\right)\left(\left\{J_{(101)}\right\}+\left\{J_{(202)}+J_{(211)}+\right.\right.$ $\left.\left.J_{(121)}+J_{(112)}\right\}\right) \subset\left\{J_{(222)}\right\}+\left\{J_{(121)}+J_{(112)}+J_{(202)}+J_{(211)}\right\}$ where $J_{(222)}=0$ by (3a); in (iii') are $L\left(J_{(110)}, J_{(101)}\right)\left(J_{(211)}+J_{(112)}+J_{(202)}\right) \subset J_{(220)}+J_{(121)}+J_{(211)}$; 
in (iv') are $L\left(J_{(211)}, J_{(121)}\right)\left(J_{(011)}+J_{(110)}\right) \subset J_{(101)}+J_{(200)}$ where $J_{(200)}=0$ again by (3a), $L\left(J_{(211)}, J_{(220)}\right) J_{(110)} \subset J_{(101)}, L\left(J_{(220)}, J_{(211)}\right) J_{(101)} \subset J_{(110)}$. Thus the conditions of 4.3 are met, and $S_{\mathscr{E}}$ is an automorphism in this case.

Now consider the case $\mathscr{E}=\{e, f\}$. We first note that conditions (2a)-(2c) imply the further conditions

$\left(2 \mathrm{a}^{\prime}-\mathrm{b}^{\prime}\right) \quad P\left(J_{(11)}\right)\left\{J_{(21)}+J_{(12)}+J_{(10)}+J_{(01)}+J_{(00)}\right\}=0$

$\left(2 \mathrm{c}^{\prime}\right) \quad P\left(J_{(10)}, J_{(01)}\right)\left\{J_{(11)}+J_{(00)}\right\}=0$

$\left(2 \mathrm{c}^{\prime \prime}\right) \quad L\left(J_{(11)}, J_{(10)}+J_{(01)}\right) J_{(00)}=0$

$\left(2 \mathrm{c}^{\prime \prime \prime}\right) \quad L\left(J_{(10)}, J_{(11)}\right) J_{(12)}=L\left(J_{(01)}, J_{(11)}\right) J_{(21)}=0$.

Indeed, (2a-b) imply $\left(2 \mathrm{a}^{\prime}-\mathrm{b}^{\prime}\right)$ since $P\left(x_{11}\right) y_{j 1}=\left\{\left\{x_{11} y_{j 1} f\right\} f x_{11}\right\}-\left\{\left(P\left(x_{11}\right) f\right) y_{j 1} f\right\}$ (by $(0.4))=0$ by $(2 \mathrm{~b})$ and $\left\{x_{11} y_{j 1} f\right\} \in J_{2-j, 2}=0$ for $j=2$ or 0 by (2a). (2c) implies $\left(2 c^{\prime}\right)$ since $\left\{x_{10} y_{00} z_{01}\right\}=\left\{x_{10}\left\{y_{00} z_{01} f\right\} f\right\}$ (by linearized (0.3) acting on $\left.x_{10}\right)=\left\{x_{10} w_{11} f\right\}=0$ by (2c), and $\left\{x_{10} y_{11} z_{01}\right\}=\left\{x_{10} e\left\{e y_{11} z_{01}\right\}\right.$ (by linearized $(0.3)$ on $\left.x_{10}\right)=0$ by (2c), (2c) implies $\left(2 c^{\prime \prime}\right)$ since $\left\{z_{00} y_{10} x_{11}\right\}=$ $\left\{z_{00}\left\{y_{10} x_{11} f\right\} f\right\}$ (by linearized (0.3)) $=0$ by (2c), and (2c) implies (2c' $\left.\mathrm{c}^{\prime \prime \prime}\right)$ since $\left\{x_{10} y_{11} z_{12}\right\}=\left\{\left\{x_{10} y_{11} f\right\} f z_{12}\right\}$ (by linearized $\left.(0.3)\right)=0$ by (2c) again. Thus we may employ all these.

To verify (4.3(i)-(iv)) for $\mathscr{E}=\{e, f\}$ it suffices by symmetry in $e, f$ and $L\left(J_{I}, J_{I}\right) J_{K} \subset J_{K}$ to verify (i'-ii') $P\left(J_{(11)}\right), P\left(J_{(10)}\right), P\left(J_{(11)}, J_{(10)}\right)$, $P\left(J_{(10)}, J_{(01)}\right), P\left(J_{(21)}\right), P\left(J_{(21)}, J_{(12)}\right)$ all leave $J_{1}$ and $J_{2}+J_{0}$ invariant, (iii') $L\left(J_{(11)}, J_{(10)}\right)$ and $L\left(J_{(10)}, J_{(11)}\right)$ and $L\left(J_{(10)}, J_{(01)}\right)$ leave $J_{2}+J_{0}$ invariant, (iv') $L\left(J_{(21)}, J_{(12)}\right)$ leaves $J_{1}$ invariant. By the Peirce relations (1.2) the only nontrivial products in $\left(\mathrm{i}^{\prime}-\mathrm{ii}^{\prime}\right)$ are $P\left(J_{(11)}\right)\left(\left\{J_{(21)}+J_{(12)}+\right.\right.$ $\left.\left.J_{(00)}\right\}+\left\{J_{(11)}+J_{(10)}+J_{(01)}\right\}\right) \subset\{0+0+0\}+\left\{J_{(11)}+0+0\right\}$ by $\left(2 a^{\prime}-b^{\prime}\right)$, $P\left(J_{(10)}\right)\left(J_{(00)}+J_{(10)}\right) \subset 0+J_{(10)}$ by $(2 \mathrm{a}), \quad P\left(J_{(11)}, J_{(10)}\right)\left(\left\{J_{(21)}+J_{(00)}\right\}+\right.$ $\left.\left\{J_{(11)}+J_{(10)}+J_{(01)}\right\}\right) \subset\left\{J_{(00)}+J_{(21)}\right\}+\left\{J_{(10)}+J_{(11)}+0\right\}$ by $(2 \mathrm{a}), P\left(J_{(10)}\right.$, $\left.J_{(01)}\right)\left(J_{(00)}+\left\{J_{(11)}+J_{(10)}+J_{(01)}\right\}\right) \subset 0+\left\{0+J_{(01)}+J_{(10)}\right\}$ by $\left(2 \mathrm{c}^{\prime}\right), P\left(J_{(21)}\right) J_{(21)} \subset$ $J_{(21)}, P\left(J_{(21)}, J_{(12)}\right)\left(\left\{J_{(21)}+J_{(12)}\right\}+\left\{J_{(11)}\right\}\right) \subset\left\{J_{(12)}+J_{(21)}\right\}+0$ by $(2 \mathrm{a}) ;$ in (iii') are $L\left(J_{(11)}, J_{(10)}\right)\left(J_{(21)}+J_{(00)}\right)=0+0$ by $(2 \mathrm{a})$ and $\left(2 \mathrm{c}^{\prime \prime}\right), L\left(J_{(10)}\right.$, $\left.J_{(11)}\right)\left(J_{(21)}+J_{(12)}\right)=0$ by $(2 \mathrm{a})$ and $\left(2 \mathrm{c}^{\prime \prime \prime}\right), L\left(J_{(10)}, J_{(01)}\right) J_{(12)} \subset J_{(21)}$; and in (iv') are $L\left(J_{(21)}, J_{(12)}\right)\left(J_{(11)}+J_{(01)}\right) \subset 0+J_{(10)}$ by (2a). Thus (2a-c) suffice to yield 4.3(i)-(iv), and $S_{\mathscr{8}}$ is an automorphism in this case too.

Rectangular Reflection Proposition 4.5. The Peirce reflection $S_{\{e, f\}}$ relative to collinear tripotents $e, f$ will be an antomorphism of $J$ if either $e, f$ imbeds in a quadrangle $\{e, f, g, h\}$ such that

(i ) $J_{(2200)}=J_{(1111)}=0$, or if e,f satisfy

(ii) $J_{(22)}=J_{(10)}=0$.

Proof. In case (i), from $J_{(2200)}=0$ we get by 3.4(i) that

$$
J_{(2002)}=P\left(e_{1}\right) J_{(2200)}, J_{(0220)}=P\left(e_{2}\right) J_{(2200)}, J_{(0022)}=P\left(e_{3}\right) P\left(e_{2}\right) J_{(2200)}
$$


also vanish and the quadrangular Peirce decomposition (3.3iv) reduces to

$$
\begin{aligned}
J= & J_{(2101)}+J_{(1210)}+J_{(1012)}+J_{(0121)}+J_{(1001)}+J_{(0110)}+J_{(1100)} \\
& +J_{(0011)}+J_{(0000)} .
\end{aligned}
$$

Relative to $e, f$ this says

$$
\begin{aligned}
& J_{(22)}=J_{(20)}=J_{(02)}=0, J_{(21)}=J_{(2101)} J_{(12)}=J_{(1210)} \\
& J_{(10)}=J_{(1012)}+J_{(1001)}, J_{(01)}=J_{(0121)}+J_{(0110)}, \\
& J_{(11)}=J_{(1100)}, J_{(00)}=J_{(0011)}+J_{(0000)} .
\end{aligned}
$$

Thus $P\left(J_{(11)}\right) e \subset P\left(J_{0}(h)\right) J_{1}(h)=0,\left\{J_{(01)} J_{(11)} e\right\}=\left\{J_{(0121)} J_{(1100)} e\right\}+\left\{J_{(0110)} J_{(1100)} e\right\} \subset$ $\left\{J_{2}(g) J_{0}(g) e\right\}+\left\{J_{(0110)} J_{(1100)} J_{(2101)}\right\} \subset 0+J_{(1111)}=0$ by hypothesis, and dually for $f$. Thus condition $(2(a-c))$ of (4.4) met, and $S_{\{e, f\}}$ is an automorphism.

In case (ii) $J_{(22)}=0$ implies $J_{(20)}=J_{(02)}=0$ by $3.4(\mathrm{i})$, and $J_{(10)}=0$ implies $J_{(01)}=0$ by (3.4ii). Since $P\left(J_{(11)}\right) e \subset J_{(01)}=0$ and $P\left(J_{(11)}\right) f \subset$ $J_{(10)}=0$, the conditions $2 \mathrm{a}-\mathrm{c}$ are met in this case too, and again $S_{\{e, f\}}$ is an automorphism.

Symplectic Reflection Proposition 4.6. The Peirce reflection $S_{|e, f, k|}$ relative to pairwise collinear tripotents $e, f, k$ will be an automorphism of $J$ if $e, f$ imbeds in a quadrangle $\{e, f, g, h\}$ so $k$ remains collinear with $g, h$, and

(i ) $J_{(2200)} \subset J_{0}(k)$

(ii) $J_{(0011)} \subset J_{0}(k)$

(iii) $J_{(1111)} \subset J_{2}(k)+J_{0}(k)$.

Proof. These 3 conditions imply the quadrangular Peirce decomposition (3.3iv) has

(i' $) J_{(2200)}+J_{(0022)} \subset J_{0}(k), J_{(2002)}+J_{(0220)} \subset J_{2}(k)$

(ii') $J_{(0011)}+J_{(1100)} \subset J_{0}(k), J_{(0110)}+J_{(1001)} \subset J_{1}(k)$

(iii') $J_{(111)} \subset J_{2}(k)+J_{0}(k)$

(iv') $J_{(2101)}+J_{(1210)}+J_{(1012)}+J_{(0121)} \subset J_{1}(k)$

$\left(v^{\prime}\right) \quad J_{(0000)} \subset J_{0}(k)$.

Indeed, from (i) we see $J_{(2002)}=P(e) J_{(2200)}$ (by $(3.4(\mathrm{i})) \subset P\left(J_{1}(k)\right) J_{0}(k) \subset$ $J_{2}(k)$ and similarly $J_{(0220)} \subset J_{2}(k)$, while $J_{(0022)}=P(g) J_{(0220)} \subset P\left(J_{1}(k)\right) J_{2}(k) \subset$ $J_{0}(k)$, yielding (i'). Also (3.4ii) shows that (i) implies $J_{(1100)}=\left\{f g J_{(0011)}\right\} \subset$ $\left\{J_{1}(k) J_{1}(k) J_{0}(k)\right\} \subset J_{0}(k), J_{(0110)} \cap J_{0}(k)=\left\{f k\left(J_{(0011)} \cap J_{1}(k)\right)\right\}=0$ and similarly $J_{(1001)} \cap J_{0}(k)=0$, while $J_{(0110)} \cap J_{2}(k)=P(k)\left\{J_{(2112)} \cap J_{2}(k)\right\}$ (using $3.4(\mathrm{i}))=0$ and $J_{(1001)} \cap J_{2}(k)=0$ automatically, so $J_{(0110)}+J_{(1001)} \subset J_{1}(k)$ by compatibility, yielding (ii'). By (3.4i) we have $J_{(2101)} \cap J_{2}(k)=$ $P(e)\left\{J_{(2101)} \cap J_{0}(k)\right\}$ and by (3.4ii) $J_{(2101)} \cap J_{0}(k)=\left\{f k\left(J_{(2002)} \cap J_{1}(k)\right)\right\}=0$ by (i'), so $J_{(2101)} \subset J_{1}(k)$ by compatibility, similarly for $J_{(1210)}$, while 
using $g, h$ in place of $e, f$ yields $J_{(0121)} \cap J_{2}(k)=J_{(0121)} \cap J_{0}(k)=0$ so $J_{(012)} \subset J_{1}(k)$ and dually for $J_{(1012)}$. Finally $\left(\mathrm{v}^{\prime}\right)$ follows from (3.3iv), since by Lemma $3.2\{e, k, g\}$ imbeds in a quadrangle. From these we immediately obtain the condition $(3 a),(3 b)$ for $\{e, f, k\}$ : for $(3 b)$ $J_{(111)}=\left\{J_{(1111)}+J_{(1100)}\right\} \cap J_{1}(k)=0$ by (iii), (ii'), $J_{(100)}=\left\{J_{(1012)}+J_{(1001)}\right\} \cap$ $J_{0}(k)=0$ by $\left(\mathrm{iv}^{\prime}\right)$, (ii'), dually $J_{(010)}=0$, and $J_{(001)}=\left\{J_{(0022)}+J_{(0011)}+\right.$ $\left.J_{(0000)}\right\} \cap J_{1}(k)=0$ by (i'), (ii'), ( $\left.\mathrm{v}^{\prime}\right)$; for (3a) $J_{(22 j)}=J_{(2200)} \cap J_{j}(k)=0$ unless $j=0$ by (i'), $J_{(21 j)}=J_{(2101)} \cap J_{j}(k)=0$ unless $j=1$ by (iv'), $J_{(20 j)}=J_{(2002)} \cap J_{j}(k)=0$ unless $j=2$ by $\left(\mathrm{i}^{\prime}\right)$, dually for $J_{(j 2 i)}$, and $J_{(002)}+J_{(102)}+J_{(012)}=\left\{J_{(0000)}+J_{(0011)}+J_{(1001)}+J_{(1012)}+J_{(0110)}+J_{(0121)}\right\} \cap$ $J_{2}(k)=0$ by $\left(\mathrm{v}^{\prime}\right),\left(\mathrm{ii}^{\prime}\right),\left(\mathrm{ii}^{\prime}\right),\left(\mathrm{iv}^{\prime}\right),\left(\mathrm{ii}^{\prime}\right),\left(\mathrm{iv}^{\prime}\right)$. Thus the hypotheses $(3 \mathrm{a}-\mathrm{b})$ of (4.4) are met, and $S_{\{e f k\}}$ is an automorphism.

EXAMPLE 4.7. If $e=E_{11}, f=E_{12}$ in $M_{p, q}(D)$ then the Peirce reflection $S_{\{e, f\}}$ is an automorphism $X \rightarrow\left(I_{p}-2 E\right) X\left(I_{q}-2 F\right)$ for $E=E_{11} \in M_{p}(D), F=E_{11}+E_{22} \in M_{q}(D)$. If $p, q \geqq 2$ then $e, f$ imbeds in a quadrangle $\{e, f, g, h\}=\left\{E_{11}, E_{12}, E_{22}, E_{21}\right\}$ as in (4.5).

EXAMPLE 4.8. If $e=F_{12}, f=F_{13}$ in $S_{n}(C)$ for $n \geqq 4$, then $S_{\{e, f\}}$ is not an automorphism if characteristic $C \neq 2: F_{23}$ and $F_{34}$ lie in $J_{1}(\mathscr{E}), F_{12}$ in $J_{2}(\mathscr{E})$, yet $\left\{F_{34} F_{23} F_{12}\right\}=-F_{14}$ lies in $J_{1}(\mathscr{E})$. Note condition $(4.4(2 \mathrm{c}))$ is violated here: $\left\{F_{34} F_{23} F_{12}\right\}=-F_{14}$ is nonzero in $\left\{J_{(01)} J_{(11)} e\right\}$. The trouble is that $C F_{23}$ really acts like part of $J_{2}$-if we take $\mathscr{C}^{\prime}=\{e, f, k\}=\left\{F_{12}, F_{13}, F_{23}\right\}$ then $S_{\{e, f, k\}}$ is an automorphism $X \rightarrow\left(I_{n}-2 E\right) X\left(I_{n}-2 E\right)$ for $E=E_{11}+E_{22}+E_{33}$ in $M_{n}(C)$. Here $e, f$ imbeds in the quadrangle $\{e, f, g, h\}=\left\{F_{12}, F_{13}, F_{43}, F_{42}\right\}$ collinear with $F_{23}$ as in (4.6.)

5. Lifting compatible families. In considering Wedderburn splittings and the second cohomology group $H^{2}(\bar{J}, M)$, it is important to be able to lift compatible covering families from $\bar{J}$ to any null extension $J$ (i.e., lifting from $\bar{J} \cong J / M$ to $J$ modulo a null or trivial ideal $M$ ). In this section we consider the general problem of lifting a compatible family modulo a nil ideal.

In lifting a family the crucial step is always lifting a single tripotent.

Lifting LemMa 5.1 (1, p. 108). If $J \stackrel{\pi}{\rightarrow} \bar{J}$ is a projection of Jordan algebras whose kernel is nil, then for each idempotent $\bar{e} \in \bar{J}$ and each preimage $x \in J(\pi(x)=\bar{e})$, there is a preimage $e=p(x)$ which is a polynomial in the given $x$ and is an idempotent in $J$. The same holds if $J, \bar{J}$ are Jordan pairs.

If $J$ and $\bar{J}$ are Jordan triple systems, then tripotents $\bar{e}$ can be 
lifted modulo nil ideals to tripotents $e=p(x)$ as long as $1 / 2 \in \Phi$.

Proof. In the polynomial ring $\Phi[t]$ we have

$$
f(g(t)) \in U(f(t)) \Phi[t] \text { for } f(t)=t-t^{2}, g(t)=3 t^{2}-2 t^{3} .
$$

Thus by induction $f\left(g^{(n)}(t)\right) \in U\left(f(t)^{2^{n-1}}\right) \Phi[t]$ and the iterates $g^{(1)}(x)$, $g^{(2)}(x), \cdots,\left(g^{(1)}(x)=g(x), g^{(m+1)}(x)=g\left(g^{(m)}(x)\right)\right)$ converge to an idempotent $e=g^{(n)}(x)$ : since $\pi(f(x))=\pi\left(x-x^{2}\right)=\bar{e}-\vec{e}^{2}=0$ we have $f(x) \in \operatorname{Ker} \pi$ nil by hypothesis, $f(x)^{2^{2-1}}=0$ for suitably large $n$, hence $f\left(g^{(n)}(x)\right)=0$ and $e=g^{(n)}(x)$ has $e-e^{2}=f(e)=0$. Thus $e$ is an idempotent. It still covers $\bar{e}$ since $\pi\left(g^{(n)}(x)\right)=g^{(n)}(\pi(x))=g^{(n)}(\bar{e})=\bar{e}$ since $g(\bar{e})=3 \bar{e}-2 \bar{e}=\bar{e}$. The Jordan algebra proof can be used to derive the result for pairs ([2, p. 109]).

In triple systems only odd powers are defined, hence we can only consider odd polynomials. When $1 / 2$ is available we have

$$
f(g(t)) \in P(f(t)) \Phi_{\text {odd }}[t] \text { for } f(t)=t-t^{3}, g(t)=5 / 2 t^{3}-3 / 2 t^{5} .
$$

As before, after a finite number of steps the iterates $g^{(n)}(x)$ converge to a tripotent, $e, e-e^{3}=f(e)=0$.

If we do not wish to wait for a sequence to converge, we can produce the idempotent lift directly. In the Jordan algebra case, suppose $x-x^{2}$ is nilpotent of index $n$. We claim

$$
g(t)-g(t)^{2} \in U\left(\left(t-t^{2}\right)^{n}\right) \Phi[t] \text { for } g(t)=\left\{1-(1-t)^{2 n}\right\}^{2 n} .
$$

Indeed, $t^{2 n}$ divides $g$ since $1-(1-t)^{2 n}$ vanishes at $t=0$ and hence is divisible by $t$, similarly $1-g=1-(1-u)^{2 n}$ (for $u=(1-t)^{2 n}$ ) is divisible by $u$, thus $g-g^{2}=g(1-g)$ is divisible by $t^{2 n} u=t^{2 n}(1-t)^{2 n}=$ $\left\{t-t^{2}\right\}^{2 n}$. Therefore $e=g(x)$ has $e-e^{2} \in U\left(\left(x-x^{2}\right)^{n}\right) J=0$, where $g(0)=0$ and $g(1)=1$ guarantee $g(x)$ makes sense in (the perhaps nonunital) $J$ and $\pi(g(x))=g(\bar{e})=g(1) \bar{e}=\bar{e}$.

The Jordan triple case is more complicated. We have

$$
g(t)-g(t)^{3} \in P\left(\left(t-t^{3}\right)^{n}\right) \Phi_{\text {odd }}[t] \text { for } g(t)=t^{2 n+1} h(s) k(s)^{n} \text { where } s=1-
$$
$t^{2}$ and $k(s)=1+s+\cdots+s^{2 n-1}$ and $h(s)=1+1 / 2 s+3 / 8 s^{2}+\cdots$ $+\alpha_{n-1} s^{2 n-1} \in Z[1 / 2][s]$ is the first $2 n$ terms of $(1-s)^{-1 / 2}=$ $k(s)^{1 / 2}$.

Clearly $g$ is an odd polynomial since $s$ is even. $g-g^{3}=g\left(1-g^{2}\right)$ is divisible by $\left\{t\left(1-t^{2}\right)\right\}^{2 n}$ since $g$ is divisible by $t^{2 n}$ and $1-g^{2}=1-$ $\left(t^{2}\right)^{2 n+1} h(s)^{2} k(s)^{2 n}$ is divisible by $\left(1-t^{2}\right)^{2 n}=s^{2 n}$ because modulo $s^{2 n}$ it is $\equiv 1-(1-s)^{2 n+1} k(s) k(s)^{2 n}=1-(1-s)^{2 n+1}\left(1+s+\cdots+s^{2 n-1}\right)^{2 n+1}=1-$ $\left(1-s^{2 n}\right)^{2 n+1} \equiv 0$. Once more the specialization $\Phi_{\text {odd }}[t] \rightarrow J$ via $t^{2 k+1} \rightarrow$ $x^{2 k+1}$ yields $e=g(x)$ with $e-e^{3}=0, \pi(e)=g(\bar{e})=g(1) \bar{e}=\bar{e}$. 
EXAMPLE 5.6. It is somewhat surprising that $1 / 2$ is necessary for lifting in triple systems, but one can give a "generic" example of a non-liftable tripotent. One can calculate that there is no integral polynomial $g(t) \in Z_{\text {odd }}[t]$ such that $g(1)=1$ and $g(t)-g(t)^{3}$ is divisible by $\left(t-t^{3}\right)^{2}$. Thus if $B=Z_{\text {odd }}[t]=t Z[s]\left(s=t^{2}\right), L=(1-s) B=$ $t(1-s) Z[s]=\left(t-t^{3}\right) Z[s], K=\left(t-t^{3}\right)^{2} B=s(1-s)^{2} B$ then $J=B / K \rightarrow$ $B / L=\bar{J}$ has nil (even trivial) kernel $L / K$, yet $\bar{e}=\bar{t}$ is a tripotent in $\bar{J}$ with no covering tripotent $e=g(t)$ in $J$. Thus lifting is not always possible in triple systems.

REMARK 5.7. The lift $e$ obtained from an arbitrary $x$ may not be the "correct" one. For example, if $e$ is the "correct" cover of $\bar{e}$ and we choose a preimage $x=e-w=e-\left(w_{2}+w_{1}+w_{0}\right)$ for $w_{i} \in K \cap J_{i}(e)$ (for trivial $K=\operatorname{Ker} \pi: J \rightarrow \bar{J}$ ), then one easily computes

$$
x^{2 n+1}=e-\left(w_{1}+w_{2}+n\left(w_{2}+w_{2}^{*}\right)\right) \quad\left(n>0, w_{2}^{*}=P(e) w_{2}, K^{2}=0\right) .
$$

Therefore $p(x)=\sum \alpha_{n} x^{2 n+1}=\left(\sum \alpha_{n}\right)\left(e-\left(w_{1}+w_{2}\right)\right)-\alpha_{0} w_{0}-\sum n \alpha_{n}\left(w_{2}+w_{2}^{*}\right)$ covers $\bar{e}$ iff $\sum \alpha_{n}=1$. In general $f=e-\left(z_{2}+z_{1}+z_{0}\right)$ is tripotent for $z_{i} \in K \cap J_{i}(e)$ iff $z_{0}=z_{2}+z_{2}^{*}=0(P(f)-f=z-P(e) z-\{e e z\}=$ $\left(z_{2}+z_{1}+z_{0}\right)-\left(z_{1}+2 z_{2}+z_{2}^{*}\right)$ by triviality of $\left.K\right)$, so $p(x)=f$ is a tripotent cover of $\bar{e}$ iff

$$
\sum \alpha_{n}=1, \alpha_{0} w_{0}=0,\left\{1+2 \sum n \alpha_{n}\right\}\left(w_{2}+w_{2}^{*}\right)=0 .
$$

In this case

$$
f=e-\left(w_{1}+1 / 2\left(w_{2}-w_{2}^{*}\right)\right) .
$$

Thus in general we cannot get rid of the components $w_{1}$ and $w_{2}$, so no lift $f \in \Phi[x]$ is the correct lift $e$.

Once we can lift a single idempotent, we can without further ado lift a countable family of orthogonal idempotents. It is not clear that we can always lift compatible families. We will be able to lift certain families intermediate between orthogonal and compatible. A linearly-ordered family $\left\{e_{\alpha}\right\}$ of tripotents is hierarchical if $\beta>\alpha$ implies $e_{\beta}$ lies in one of the Peirce spaces $J_{i}\left(e_{\alpha}\right)$. An important special case is that of an orthogonal family $\left(e_{\beta} \in J_{0}\left(e_{\alpha}\right)\right)$ or a collinear family $\left(e_{\beta} \in J_{1}\left(e_{\alpha}\right)\right)$, or more generally an orthogonal-collinear family where any two $e_{\alpha}, e_{\beta}$ are either orthogonal or collinear. It is easy to see by (1.8(iii)) that any hierarchical family is compatible.

Countable Lifting Proposition 5.8. If $J \stackrel{\pi}{\rightarrow} \bar{J}$ is a projection of Jordan algebras with nil kernel, then any finite or countable hierarchical family $\bar{e}_{1}, \bar{e}_{2}, \ldots$ of idempotents in $\bar{J}$ can be lifted to 
a hierarchical family $e_{1}, e_{2}, \cdots$ of idempotents in $J$. If $J$ and $\bar{J}$ are unital and $\bar{e}_{1}, \cdots, \bar{e}_{n}$ are supplementary orthogonal idempotents in $\bar{J}$, then $e_{1}, \cdots, e_{n}$ are supplementary orthogonal idempotents in $J$. The same results hold if $J, \bar{J}$ are Jordan pairs.

If $J \stackrel{\pi}{\rightarrow} \bar{J}$ is a projection of Jordan triple systems with nil kernel, and if $1 / 2 \in \Phi$, then any finite or countable hierarchical family of tripotents in $\bar{J}$ can be lifted to a hierarchical family in $J$.

Proof. Assume $\left\{\bar{e}_{i}\right\}$ is a hierarchical family of tripotents (resp. idempotents in the Jordan algebra case). By the Lifting Lemma 5.1 we can under our hypotheses lift $\bar{e}_{1}$ to $e_{1}$, which is by itself trivially hierarchical. Assume we have lifted $\left\{\bar{e}_{1}, \cdots, \bar{e}_{n}\right\}$ to hierarchical $\left\{e_{1}, \cdots, e_{n}\right\}$. Then these are in particular compatible, and determine a Peirce decomposition $J=\bigoplus J_{\left(i_{1}, \ldots, i_{n}\right)}$ as in (2.1), with $\pi\left(J_{\left(i_{1}, \cdots, i_{n}\right)}\right)=\bar{J}_{\left(i_{1}, \cdots, i_{n}\right)}$ relative to $\bar{e}_{1}, \cdots, \bar{e}_{n}$. Now by hierarchy $\bar{e}_{n+1}$ lies in some single Peirce space $\bar{J}_{\varepsilon_{i}}\left(\bar{e}_{i}\right)$ relative to each $\bar{e}_{1}, \cdots \bar{e}_{n}$, so $\bar{e}_{n+1} \in \bar{J}_{\left(\varepsilon_{1}, \ldots, \varepsilon_{n}\right)}$. Then we can choose a preimage $x$ of $\bar{e}_{n+1}$ lying in $J_{\left(\varepsilon_{1}, \cdots, \varepsilon_{n}\right) \cdots}$ As a result the tripotent (resp. idempotent) $e_{n+1}=p(x)$ given by (5.1) automatically stays inside the sub-triple system $J_{\left(\varepsilon_{1}, \cdots, \varepsilon_{n}\right)}=$ $\cap J_{\varepsilon_{i}}\left(e_{i}\right)$, so $e_{n+1}$ automatically lies in $J_{\varepsilon_{i}}\left(e_{i}\right)$ for each $i=1,2, \cdots, n$, and hence $\left\{e_{1}, e_{2}, \cdots, e_{n}, e_{n+1}\right\}$ is again hierarchical.

If $\bar{e}_{1}, \cdots, \bar{e}_{n}$ are supplementary in $\bar{J}$ then in $J$ the idempotent $e=e_{1}+\cdots+e_{n}$ (using orthogonality!) covers $\overline{1}, \pi(e)=\bar{e}_{1}+\cdots+$ $\bar{e}_{n}=\overline{1}$. Thus $\pi(1-e)=0,1-e \in \operatorname{Ker} \pi$ is nil, yet at the same time $1-e$ is idempotent. Thus $1-e=0$ and $e_{1}+\cdots+e_{n}=e=1$ are supplementary in $J$.

OPEN QUESTION 5.9. Can we lift arbitrary compatible families? Can strongly compatible families, at least, be lifted to compatible families? Can orthogonal-collinear families be lifted to orthogonalcollinear families? (By the above, orthogonal-collinear $\left\{\bar{e}_{i}\right\}$ can be lifted to hierarchical $\left\{e_{i}\right\}$, so orthogonality $\bar{e}_{\alpha} \perp \bar{e}_{\beta} \Leftrightarrow \bar{e}_{\beta} \in \bar{J}_{0}\left(\bar{e}_{\alpha}\right)$ is inherited by $e_{\alpha}, e_{\beta}$, but collinearity $\bar{e}_{\alpha} T \bar{e}_{\beta}$ is transformed only into $e_{\beta} \in J_{1}\left(e_{\alpha}\right)$, which does not quite imply collinearity $e_{\alpha} \in J_{1}\left(e_{\beta}\right)$ as well).

We can always lift two collinear tripotents modulo nilpotent ideals.

Proposition 5.10. If $J \stackrel{\pi}{\rightarrow} \bar{J}$ has Penico-solvable kernel, and $1 / 2 \in \Phi$, then any two collinear tripotents $\bar{e}, \bar{f}$ in $\bar{J}$ can be lifted to collinear tripotents $e, f$ in $J$. 
Proof. Penico solvability means $S^{n}(K)=0$ for some $n$, where $S(K)=P(J) P(K) J+P(K) J+L(K, K) J, S^{n}(K)=S\left(S^{n-1}(K)\right) . \quad$ Вy induction it suffices to consider the case $S(K)=0$ of a trivial ideal. By 5.8 we can left $\bar{e}$ to $e$, then $\bar{f} \in \bar{J}_{1}(\bar{e})$ to $f \in J_{1}(e)$. Since $f$ covers $\bar{f}, P(\bar{f}) \bar{e}=L(\bar{f}, \bar{f}) \bar{e}-\bar{e}=0$ implies $P(f) e=z_{0}, L(f, f) e-e=z_{2}$ lie in $K$, so the result follows from

LEMMA 5.11. If $1 / 2 \in \Phi$ and $e, f$ are tripotents which are collinear modulo a trivial ideal $K$ and have

$$
f \in J_{1}(e), P(f) e=z_{0}, L(f, f) e-e=z_{2} \quad\left(z_{i} \in K \cap J_{i}(e)\right)
$$

then $f^{\prime}=f-1 / 2 z_{1}$ for $z_{1}=\left\{e f z_{0}\right\}$ is a tripotent collinear with e and congruent modulo $K$ to $f$. If $g$ is a tripotent with $e \in J_{i}(g), f \in J_{j}(g)$ then $f^{\prime}$ remains in $J_{j}(g)$.

Proof. Clearly $f^{\prime}$ is congruent modulo $z_{1} \in K$ to $f$, it remains in $J_{1}(e)$ since $z_{1} \in J_{1}(e)$, and if $e \in J_{i}(g), f \in J_{j}(g)$ then by (1.2) $z_{1}=$ $L(e, f) P(f) e \in J_{j}(g)$ so $f^{\prime}$ remains in $J_{j}(g)$. We must show (i) $f^{\prime}$ is tripotent, (ii) $e \in J_{1}\left(f^{\prime}\right)$, i.e., $\left\{f^{\prime} f^{\prime} e\right\}=e$.

By triviality of $K$ we have $f^{\prime 3}=f^{3}-1 / 2\{P(f)+L(f, f)\} z_{1}=f-$ $1 / 2\{P(f)+L(f, f)\} L(e, f) z_{0}=f-1 / 2 z_{1}=f^{\prime}$ since $\{P(f)+L(f, f)\} L(e$, $f) z_{0}=\{P(P(f) e, f)+L(e, f) L(f, f)+L(\{f f e\}, f)-L(e,\{f f f\})\} z_{0}$ (by $(0.2),(0.5))=\left\{P\left(z_{0}, f\right)+L(e, f) L(f, f)+L\left(e+z_{2}, f\right)-2 L(e, f)\right\} z_{0}=$ $L(e, f)\{L(f, f)-I\} z_{0}$ (by triviality of $\left.K\right)=L(e, f) z_{0}$ because $L(f, f) z_{0}=$ $L(f, f) P(f) e=P(P(f) f, f) e \quad($ by $\quad(0.2))=2 P(f) e=2 z_{0}$. Thus $f^{\prime}$ is tripotent.

Again by triviality, $\left\{f^{\prime} f^{\prime} e\right\}=\{f f e\}-1 / 2\left[\left\{f z_{1} e\right\}+\left\{z_{1} f e\right\}\right]=e+z_{2}-$ $1 / 2\{P(e, f)+L(e, f)\} L(e, f) z_{0}=e$ since $\{P(e, f)+L(e, f)\} L(e, f) z_{0}=$ $\{L(e, e) P(f)+P(e, P(f) e)+L(P(e) f, f)+2 P(e) P(f)\} z_{0}$ (by $\left.(0.7),(0.6)\right)=$ $\left\{L(e, e) P(f)+P\left(e, z_{0}\right)+0+2 P(e) P(f)\right\} z_{0}=\{L(e, e)+2 P(e)\} P(f) z_{0} \quad$ (by triviality of $K$ again $)=2\{I+P(e)\} P(f)^{2} e\left(\right.$ as $L(e, e)=2 I$ on $\left.J_{2}(e)\right)=$ $2\left\{P(f)^{2} P(e)+P(e) P(f)^{2}\right\} e=2\left\{P(\{f f e\})+P\left(P(f)^{2} e, e\right)-L(f, f) P(e) L(f\right.$, $f)\} e($ by $(0.8))=2\left\{P\left(e+z_{2}\right)+2 P(f)^{2}-L(f, f)^{2}\right\} e$ (always $P(e)\left\{a_{1} b_{1} e\right\}=$ $\left.\left\{b_{1} a_{1} e\right\}\right)=2\left\{\left(e+2 z_{2}\right)+2 P(f)^{2} e-L(f, f) e-2 P(f)^{2} e\right\}$ (by triviality and $(0.6))=2\left\{\left(e+2 z_{2}\right)-\left(e+z_{2}\right)\right\}=2 z_{2}$. Thus $e \in J_{1}\left(f^{\prime}\right)$ is collinear with $f^{\prime}$.

However, it is not clear that this argument can be extended to show a whole collinear (or orthogonal-collinear) family can be lifted to one of the same type.

REMARK 5.12. The fact that collinear $\bar{e}, \bar{f}$ have been lifted to tripotents $e, f$ with $f \in J_{1}(e)$ does not imply collinearity $e \in J_{1}(f)$, hence the modification $f^{\prime}$ in the above proof is really necessary. 
For example, take $e=1[12], f=1[13]+\varepsilon \delta[23]$ in $J=H_{3}(\Omega[\varepsilon])$ for $\Omega$ having nontrivial involution with $\delta \in \Omega$ having trace $t(\delta)=1, \varepsilon^{2}=$ $0, K=\varepsilon J$. Then $f \in J_{1}(e)$ but $z_{0}=P(f) e=t(\varepsilon \delta) 1[33]=\varepsilon[33], \quad z_{2}=$ $\{f f e\}-e=t\left(\varepsilon \delta^{*}\right)[11]+t(\varepsilon \delta)[22]=\varepsilon(1[11]+1[22])$. The modification obtained above is $f^{\prime}=f-1 / 2\left\{e f z_{0}\right\}=f-1 / 2 \varepsilon[23]=1[13]+\varepsilon \eta[23]$ for $\eta=\delta-1 / 2$ with $t(\eta)=0$. We never recover the "correct" modification $f-\varepsilon \delta[23]=1[13]$ this way.

EXAMPLE 5.13. Strongly compatible families cannot in general be lifted to strongly compatible families. For example, take $J=$ $\Omega e_{11}+\Omega e_{12}+\varepsilon \Omega e_{13}+\varepsilon \Omega e_{21}+\varepsilon \Omega e_{22}+\Omega e_{23} \subset M_{2,3}(\Omega)$ (the $2 \times 3$ matrices over $\Omega=\Phi[\varepsilon], \varepsilon^{2}=0$, via $\left.P(x) y=x y^{t} x\right)$. Note here $J$ has the form $J=$ $J_{0}\left(e_{23}\right)+\varepsilon J_{1}\left(e_{23}\right)+J_{2}\left(e_{23}\right)$ in $M_{2,3}(\Omega)$, and hence automatically is a subtriple system. Then $K=\varepsilon M_{2,3}(\Omega)$ is a trivial ideal, $\bar{e}=\bar{e}_{11}$ and $\bar{f}=\bar{f}_{1} \oplus \bar{f}_{0}=\bar{e}_{12} \oplus \bar{e}_{23}$ are strongly compatible in $J / K \cong\left\{\Phi e_{11}+\Phi e_{12}\right\} \boxplus$ $\left\{\Phi e_{23}\right\}$, but no covers $e=e_{11}+\varepsilon r, f=e_{12}+e_{23}+\varepsilon s$ are strongly compatible since $P(f) L(e, e) \varepsilon e_{13}=\varepsilon e_{22}, L(e, e) P(f) \varepsilon e_{13}=0$.

6. Wedderburn Splittings and $H^{2}$. Another case where the Peirce orthogonality relations (2.5) suffice is in showing that Wedderburn splittings of direct sums can be reduced to splittings of the individual factors. Recall $\left[6\right.$, p. 286] that $H^{2}(J, M)$ denotes the equivalence classes of null extensions $0 \rightarrow M \stackrel{i}{\rightarrow} \widetilde{J} \stackrel{\pi}{\rightarrow} J \rightarrow 0$ of $J$ by a bimodule $M . \quad H^{2}(J, M)=0$ iff all extensions split, in the sense that there exist homomorphisms $\sigma: J \rightarrow \widetilde{J}$ with $\pi \circ \sigma=1_{J}$, i.e., there exists a subsystem $B=\sigma(J) \subset \widetilde{J}$ isomorphic under $\pi$ to $J$.

Proposition 6.1. If $J_{1}, \cdots, J_{n}$ are locally unital Jordan triple systems with the property that all null extensions are split, then the direct sum $J_{1} \boxplus \cdots \boxplus J_{n}$ has the same property. In terms of cohomology, if $H^{2}\left(J_{i}, M_{i}\right)=0$ for all $J_{i}$-bimodules $M_{i}$ then $H^{2}(J, M)=$ 0 for all bimodules $M$ for the direct sum $J$.

Proof. By induction it suffices to prove this for the case of two summands, recalling by (2.3) that direct sums remain locally unital. So let $\widetilde{J}$ be a null extension of $J=K \boxplus L$ by a bimodule $M: 0 \rightarrow M \rightarrow \widetilde{J} \stackrel{\pi}{\rightarrow} J \rightarrow 0$ is exact. Then $0 \rightarrow M \rightarrow \widetilde{K} \stackrel{\pi}{\rightarrow} K \rightarrow 0$ is exact for $\widetilde{K}=\pi^{-1}(K) \supset \pi^{-1}(0)=M$, and by our hypothesis on $K$ this splits: there is a subsystem $B \subset \widetilde{K} \subset \widetilde{J}$ isomorphic under $\pi$ to $K$. If $\mathscr{E}$ is a covering family for the locally unital $K$, we lift it via the given isomorphism to a covering family for $B$. Regarded as a compatible (non-covering) family in $\widetilde{J}$, it determines by (2.2) a Peirce decomposition $\widetilde{J}=\widetilde{J}_{2}(\mathscr{E}) \oplus \widetilde{J}_{1}(\mathscr{E}) \oplus \widetilde{J}_{0}(\mathscr{E})$ with $B \subset \widetilde{J}_{2}(\mathscr{E})$. Furthermore, since 
$J=\pi(\widetilde{J})=\pi\left(\widetilde{J}_{2}\right)+\pi\left(\widetilde{J}_{1}\right)+\pi\left(\widetilde{J}_{0}\right)=J_{2}(\mathscr{E}) \oplus J_{1}(\mathscr{E}) \oplus J_{0}(\mathscr{E})$ where $L$ is orthogonal to $\mathscr{E} \subset K$, we must have $\pi\left(\widetilde{J}_{2}\right)=J_{2}(\mathscr{E})=K, \pi\left(\widetilde{J}_{0}\right)=$ $J_{0}(\mathscr{E})=L, \pi\left(\widetilde{J}_{1}\right)=J_{1}(\mathscr{E})=0$. Thus $0 \rightarrow M_{0}(\mathscr{E}) \rightarrow \widetilde{J}_{0}(\mathscr{E}) \stackrel{\pi}{\rightarrow} L \rightarrow 0$ is exact, hence by our hypothesis on $L$ it too splits: there is a subsystem $C \subset \widetilde{J}_{0}(\mathscr{C})$ isomorphic under $\pi$ to $L$. Because we have lifted $B$ into $\widetilde{J}_{2}(\mathscr{E})$ and $C$ into $\widetilde{J}_{0}(\mathscr{E})$, they are automatically orthogonal by Peirce orthogonality (2.2) (P1), and the sum $B \boxplus C \subset \widetilde{J}$ is automatically a direct sum of triple systems isomorphic under $\pi$ to $K \boxplus$ $L=J$. Thus $\widetilde{J}=(B \oplus C) \oplus M$ is split.

The argument actually shows it suffices if all but one of the $J_{i}$ is locally unital, since we never needed a cover for $L$.

The crucial step in the above proof was lifting a cover $\mathscr{E}$ of $K$ to a compatible family in $J$, which depended on knowing $H^{2}(K, M)=$ 0 . To get a relation between $H^{2}(J, M)$ and the $H^{2}\left(J_{i}, M_{i}\right)$ in general, we need to be able to lift the tripotents which form the local unit of $J_{i}$. This is why we studied the problem of lifting compatible families modulo trivial ideals in (5.8.)

Using hierarchical families we can reduce extensions of direct sums to extensions of the pieces. We recall how the equivalence classes of extensions of a Jordan triple system by a bimodule $M$ gains its algebraic structure $H^{2}(J, M)$. If $J$ is projective as $\Phi$ module, each extension has the form $\widetilde{J}=\sigma(J) \oplus M$ for some linear lifting $\sigma$ of $J$ into $\widetilde{J}$, and is associated with a cocycle or factor set $\rho \in C^{2}(J, M)$

$$
\rho(a ; b)=P(\sigma(a)) \sigma(b)-\sigma(P(a) b)
$$

which measures how far $\sigma$ is from being a triple system lift. The equivalence class of $\rho$ modulo coboundaries $\delta g \in B^{2}(J, M)$

$$
\delta g(a ; b)=p(a) g(b)+l(a, b) g(a)-g(P(a) b)
$$

for a linear map $g: J \rightarrow M$ is independent of the particular $\sigma$, and the extensions of $J$ by $M$ are in 1-1 correspondence with $H^{2}(J, M)=$ $C^{2}(J, M) / B^{2}(J, M)$. Thus $H^{2}(J, M)$ becomes a module over $\Phi[6, \mathrm{p}$. 287-288].

6.2. Direct Decomposition Theorem for $H^{2}$. If $J=J_{1} \boxplus \cdots \boxplus J_{n}$ is projective as module over $\Phi$ (containing 1/2), and as triple system is a direct sum of locally unital Jordan triple systems $J_{i}$ possessing hierarchical covers $\mathscr{E}_{i}$, then for any J-bimodule $M$

$$
H^{2}(J, M) \cong \oplus H^{2}\left(J_{i}, M_{i}\right)
$$

for $M_{i}=M_{i i}+M_{i 0}+M_{00}=\bigcap_{j \neq i} M_{0}\left(\mathscr{E}_{j}\right)$. The same holds for Jordan 
pairs over arbitrary $\Phi$.

Proof. We begin by imbedding $\oplus H^{2}\left(J_{i}, M_{i}\right)$ in $H^{2}(J, M)$. We have a linear map $\oplus C^{2}\left(J_{i}, M_{i}\right) \rightarrow C^{2}(J, M)$ sending $\oplus \rho_{i}$ to $\rho=\bigoplus \rho_{i}$ defined as $\rho(a ; b)=\sum \rho_{i}\left(a_{i} ; b_{i}\right)$. This induces $\oplus C^{2}\left(J_{i}, M_{i}\right) \rightarrow H^{2}(J, M)$. To characterize the kernel of this map, assume $\rho=\delta g \in B^{2}(J, M)$ for some linear $g: J \rightarrow M$. Using the Peirce decomposition $M=\bigoplus M_{j k}$ of (2.10) relative to the $\mathscr{E}_{j}$, we can write the restriction of $g$ to $J_{i}$ as $g=\bigoplus g_{j k}$ for $g_{j k}: J_{i} \rightarrow M_{j k}$. Applying the Peirce projection $E_{i i}+E_{i 0}+E_{00}$ of $M$ on $M_{i}$ to the relation $\rho\left(a_{i} ; b_{i}\right)=\delta g\left(a_{i} ; b_{i}\right)$ for $a_{i}, b_{i} \in J_{i}=J_{i i}$, we get by the Peirce relations $\rho_{i}\left(a_{i} ; b_{i}\right)=p\left(a_{i}\right)\left(g_{i i}+\right.$ $\left.g_{i 0}\right)\left(b_{i}\right)+l\left(a_{i}, b_{i}\right)\left(g_{i i}+g_{i 0}\right)\left(b_{i}\right)-\left(g_{i i}+g_{i 0}+g_{00}\right)\left(P\left(a_{i}\right) b_{i}\right)$, so that $\rho_{i}=\delta g_{i}$ for $g_{i}=g_{i i}+g_{i 0}+g_{00}: J_{i} \rightarrow M_{i}$. Conversely, if each $\rho_{i}=\delta g_{i}$ is a coboundary of $g_{i}: J_{i} \rightarrow M_{i}$ then $\rho=\bigoplus \rho_{i}$ is the coboundary $\rho=\delta g$ of $g=\bigoplus g_{i}: J \rightarrow M$. Indeed, $\delta g(a ; b)=p(a) g(b)+l(a, b) g(a)-g(P(a) b)=$ $\left\{\sum p\left(a_{i}\right)+p\left(a_{i}, a_{k}\right)\right\} \sum g_{j}\left(b_{j}\right)+\sum l\left(a_{i}, b_{i}\right) g_{j}\left(b_{j}\right)-\sum g_{i}\left(P\left(a_{i}\right) b_{i}\right)$ (by orthogonality and local unitality of $J_{i}, J_{j}$ in $\left.J\right)=\sum\left\{p\left(a_{i}\right) g_{i}\left(b_{i}\right)+l\left(a_{i}\right.\right.$, $\left.\left.b_{i}\right) g_{i}\left(a_{i}\right)-g_{i}\left(P\left(a_{i}\right) b_{i}\right)\right\}$ (since $g_{j}\left(b_{j}\right) \in M_{j} \subset M_{0}\left(\mathscr{E}_{i}\right), a_{i}, b_{i} \in J_{i} \subset J_{2}\left(\mathscr{C}_{i}\right)$ for $i \neq j)=\sum \delta g_{i}\left(a_{i} ; b_{i}\right)=\sum \rho_{i}\left(a_{i} ; b_{i}\right)=\rho(a ; b)$. Thus the kernel of the natural map $\oplus C^{2}\left(J_{i}, M_{i}\right) \rightarrow H^{2}(J, M)$ is precisely $\oplus B^{2}\left(J_{i}, M_{i}\right)$, so we have an induced imbedding of $\oplus H^{2}\left(J_{i}, M_{i}\right)=\left\{\oplus C^{2}\left(J_{i}, M_{i}\right)\right\} /\left\{\oplus B^{2}\left(J_{i}, M_{i}\right)\right\}$ in $H^{2}(J, M)$.

To see this map is surjective, i.e., that each cocycle $\rho$ is equivalent to one $\oplus \rho_{i}$ supported on the distinct $J_{i}$ with no "mixed terms", we need to choose a linear lift $\sigma$ taking $J_{i}$ into orthogonal pieces. So consider an arbitrary extension $\widetilde{J}$ of $J$ by $M$; since the kernel $M$ of $\widetilde{J} \stackrel{\pi}{\rightarrow} J$ is trivial, we can by (5.8) lift the family $\mathscr{E}=$ $\mathscr{E}_{1} \cup \cdots \cup \mathscr{E}_{n}\left(\mathscr{E}_{i}\right.$ the given hierarchical covers of $\left.J_{i}\right)$ to a hierarchical family $\tilde{\mathscr{E}}=\tilde{\mathscr{E}}_{1} \cup \cdots \cup \tilde{\mathscr{E}}_{n}$. In the associated Peirce decomposition (2.5) of $\widetilde{J}$ we have $\widetilde{J}_{i}=\widetilde{J}_{i i} \oplus \widetilde{J}_{i 0} \oplus \widetilde{J}_{00}=\bigcap_{j \neq i} \widetilde{J}_{0}\left(\widetilde{\mathscr{E}}_{j}\right)$ a subtriple system, projecting onto $\pi\left(\widetilde{J}_{i}\right)=\cap J_{0}\left(\mathscr{E}_{j}\right)=J_{i}$ with kernel $M_{i}$, and at the same time $\widetilde{J}_{i i}$ projects onto $\pi\left(\widetilde{J}_{i i}\right)=J_{i i}=J_{i}$ with kernel $M_{i i}$ (but $\widetilde{J}_{i i}$ is merely a subspace, not a subsystem), while $\widetilde{J}_{i 0}=M_{i 0}$, $\widetilde{J}_{00}=M_{00}$ since $\pi\left(\widetilde{J}_{i 0}\right)=J_{i 0}=0, \pi\left(\widetilde{J}_{00}\right)=J_{00}=0$. Since $J$ is projective over $\Phi$, so are its direct summands $J_{i}$, hence the exact sequence $0 \rightarrow M_{i i} \rightarrow \widetilde{J}_{i i} \rightarrow J_{i} \rightarrow 0$ splits as $\Phi$-module: $\widetilde{J}_{i i}=\sigma_{i}\left(J_{i}\right) \oplus M_{i i}$ for some linear lift $\sigma_{i}$. Then $\widetilde{J}_{i}=\widetilde{J}_{i i} \oplus \widetilde{J}_{i 0} \oplus \widetilde{J}_{00}=\left\{\sigma_{i}\left(J_{i}\right) \oplus M_{i i}\right\} \oplus M_{i 0} \oplus M_{00}=$ $\sigma_{i}\left(J_{i}\right) \oplus M_{i}$, and we have a linear lift $\sigma=\bigoplus \sigma_{i}$ so that $\widetilde{J}=\sigma(J) \oplus M$ with the crucial property that $\sigma\left(J_{i}\right) \subset \widetilde{J}_{i i}$ is automatically orthogonal to $\sigma\left(J_{j}\right) \subset \widetilde{J}_{j j}$. (To obtain this it is not enough to split $0 \rightarrow M_{i} \rightarrow$ $\widetilde{J}_{i} \rightarrow J_{i} \rightarrow 0$; we must work with the linear space $\widetilde{J}_{i i}$ instead of the subsystem $\widetilde{J}_{i}$.) The cocycle $\rho$ associated to $\widetilde{J}$ via this lift $\sigma$ is of the desired form $\oplus \rho_{i}$ since $\rho(a ; b)=P(\sigma(a)) \sigma(b)-\sigma(P(a) b)=$ 
$P\left(\sum \sigma_{i}\left(a_{i}\right)\right) \sum \sigma_{j}\left(b_{j}\right)-\sum \sigma_{i}\left(P\left(a_{i}\right) b_{i}\right)\left(\right.$ by $J_{i} \perp J_{j}$ in $\left.J\right)=\sum\left\{P\left(\sigma_{i}\left(a_{i}\right)\right) \sigma_{i}\left(b_{i}\right)-\right.$ $\left.\sigma_{i}\left(P\left(a_{i}\right) b_{i}\right)\right\} \quad$ by $\widetilde{J}_{i i} \perp \widetilde{J}_{j j}$ in $\left.\widetilde{J}\right)=\sum \rho_{i}\left(a_{i} ; b_{i}\right)$ for $\rho_{i} ; J_{i} \rightarrow M_{i}$ (note $\sigma_{i}\left(J_{i}\right) \subset \widetilde{J}_{i i}$, but $\widetilde{J}_{i i}$ is not in general a subsystem so the products $P\left(\sigma_{i}\left(a_{i}\right)\right) \sigma_{i}\left(b_{i}\right)$ fall in the enveloping subsystem $\widetilde{J}_{i}=\widetilde{J}_{i i}+\widetilde{J}_{i 0}+\widetilde{J}_{00}$, hence $\sigma_{i}$ falls in $\left.\widetilde{J}_{i} \cap M=M_{i}\right)$. This shows $\oplus H^{2}\left(J_{i}, M_{i}\right) \rightarrow H^{2}(J, M)$ is surjective, hence a linear isomorphism.

Note that the theorem does not depend on the hierarchical nature of the covers $\mathscr{E}_{i}$, merely on the fact that they can be lifted to compatible covers $\tilde{\mathscr{E}}_{i}$. The same result will apply to any class of liftable compatible covers.

Since the semisimple finite-dimensional Jordan triples over an algebraically closed field of characteristic $\neq 2$, or semisimple Jordan pairs with d.c.c. over an arbitrary ring of scalars, are direct sums of simple locally unital systems with hierarchical covers (even orthogonal-collinear covers, by (3.16)) we have as an immediate corollary

6.3. Direct Decomposition Theorem for $H^{2}$. If $J=J_{1} \boxplus \cdots \boxplus J_{n}$ is the decomposition into simple ideals of a semisimple Jordan pair with d.c.c. (or a semisimple Jordan triple system finite-dimensional over an algebraically closed field of characteristic $\neq 2$ ), then for any $J$-bimodule $M$ we have

$$
H^{2}(J, M) \cong \bigoplus H^{2}\left(J_{i}, M_{i}\right)
$$

for $M_{i}=M_{i \imath}+M_{i 0}+M_{00}=\bigcap_{j \neq i} M_{0}\left(\mathscr{E}_{j}\right)$ for compatible covers $\mathscr{E}_{j}$ of $J_{j}$.

This reduces the cohomology of semisimple systems to that of simple systems. Next we turn to a similar program for $H^{1}(J, M)$.

7. Derivations and structure algebras. Recall that the structural transformations $\operatorname{Strl}(J, M)$ of a Jordan triple system in a bimodule $M$ consist of all pairs $\left(S, S^{*}\right)$ of linear transformations $J \rightarrow M$ such that

$$
S(P(x) y)+P(x) S^{*}(y)=L(x, y) S(x)
$$

and similarly for $S^{*}$ with $S^{* *}=S$. The derivations Der $(J, M)$ of $J$ in $M$ are those $\left(D, D^{*}\right) \in \operatorname{Strl}(J, M)$ with $D^{*}=-D$, i.e., those linear transformations $D$ satisfying

$$
D(P(x) y)=\{D(x) y x\}+P(x) D(y) .
$$

(If $S^{*}$ is uniquely determined by $S$, e.g., if $J$ has unit, then it is 
usual to simply refer to $S$ itself as a structural transformation.) The inner structural $S$ are those in the subspace Instrl $(J, M)=$ $L(M, J)+L(J, M)$ (note by $(0.4) L(x, m)^{*}=L(m, x)$ ), the inner derivations are Inder $(J, M)=\operatorname{Instrl}(J, M) \cap \operatorname{Der}(J, M)$. This includes all sums of standard inner derivations $D(x, m)=L(x, m)-L(m, x)$, and coincides with these if $1 / 2 \in \Phi$.

Even for unital Jordan algebras, the derivations from a direct sum are not simply the sums of derivations defined on the individual pieces: the derivations on the pieces can be glued together to form a global derivation only if they are suitably orthogonal.

Proposition 7.3. A linear transformation $S: J \rightarrow M$ of a Jordan triple $J=J_{1} \boxplus \cdots \boxplus J_{n}$ into a bimodule $M$ is structural iff it has the form $S=S_{1} \oplus \cdots \oplus S_{n}$ for $S_{i}: J_{i} \rightarrow M$ satisfying the following relations (for $x_{i}, y_{i}, z_{i} \in J_{i}$ with $i, j, k \neq$ ):

(i) each $S_{i}$ is structural: $S_{i} \in \operatorname{Strl}\left(J_{i}, M\right)$

(ii) $P\left(x_{i}\right) S_{j}^{*}\left(y_{j}\right)=\left\{x_{i} y_{j} S_{i}\left(x_{i}\right)\right\}$

(iii) $\left\{x_{i} S_{j}^{*}\left(y_{j}\right) z_{k}\right\}=\left\{x_{i} y_{j} S_{k}\left(z_{k}\right)\right\}+\left\{z_{k} y_{j} S_{i}\left(x_{i}\right)\right\}$

(iv) $\left\{x_{i} S_{i}^{*}\left(y_{i}\right) z_{k}\right\}=\left\{x_{i} y_{i} S_{k}\left(z_{k}\right)\right\}+\left\{z_{k} y_{i} S_{i}\left(x_{i}\right)\right\}$.

$S$ is a derivation iff each $S_{i}$ is a derivation, $S_{i}^{*}=-S_{i}$.

Proof. The linear maps $S: J \rightarrow M$ are precisely the $S=\bigoplus S_{i}$ for $S_{i}: J_{i} \rightarrow M$. The structural condition (7.1) for all $x, y \in J$ reduces to the following conditions on the spanning elements $x_{i}, y_{i}, z_{i} \in J_{i}$ :

$$
\begin{gathered}
S_{i}\left(P\left(x_{i}\right) y_{j}\right)+P\left(x_{i}\right) S_{j}^{*}\left(y_{j}\right)=\left\{x_{i} y_{j} S_{i}\left(x_{i}\right)\right\} \\
S_{i}\left(\left\{x_{i} y_{j} z_{k}\right\}\right)+\left\{x_{i} S_{j}^{*}\left(y_{j}\right) z_{k}\right\}=\left\{x_{i} y_{j} S_{k}\left(z_{k}\right)\right\}+\left\{z_{k} y_{j} S_{i}\left(x_{i}\right)\right\}
\end{gathered}
$$

for all $i, j, k$ with $i \neq k$. Condition (7.1a) for $j=i$ is the condition (i) that $S_{i}$ be structural on $J_{i}$; for $j \neq i$, by orthogonality $J_{i} \perp J_{j}$ the condition (7.1a) reduces to (ii). Similarly, by orthogonality (7.1b) reduces to (iii) if $j \neq i, k$ and to (iv) if $j=i$.

To see that the requisite orthogonality is not automatic, consider the following

EXAMPLE 7.4. Let $J=\Phi z \boxplus \Phi w$ be a trivial Jordan triple system and $M=\Phi m \oplus \Phi n$ the bimodule determined by $\rho(z)=$ $\rho(z, w)=l(z, z)=l(w, w)=l(w, z)=0, \quad l(z, w) m=n, \quad l(z, w) n=0$. Here the split null extension $\widetilde{J}=J \oplus M$ may be imbedded in $M_{4}(\Phi)^{+}$ via $z \rightarrow e_{12}, w \rightarrow e_{23}, m \rightarrow e_{34}, n \rightarrow e_{14}$. Then $\{z w M\}=\Phi n \neq 0$, so $\left\{J_{i} J_{j} M\right\}$ is not necessarily 0 in a direct $\operatorname{sum} J=J_{1} \boxplus \cdots \boxplus J_{n}$.

Thus orthogonality of $J_{i}, J_{j}$ in $J$ does not imply orthogonality 
in all extensions: the orthogonality may be "fortuitous". However, there is an important case when orthogonality is "intrinsic", persisting in all extensions:

$\left\{J_{i} J_{j} \widetilde{J}\right\}=0$ if $J_{i}, J_{j}$ are orthogonal and one is locally unital since for example if $J_{i}$ has cover $\mathscr{E}_{i}$ then $J_{i}=J_{2}\left(\mathscr{E}_{i}\right)$ and by orthogonality $J_{j} \subset J_{0}\left(\mathscr{E}_{i}\right)$, so that $\left\{J_{i} J_{j} \widetilde{J}\right\} \subset\left\{\widetilde{J}_{2} \widetilde{J}_{0} \widetilde{J}\right\}=0$ by (2.2) (P1).

Thus if all $J_{i}$ are locally unital, (7.3ii-iv) reduce to (ii) $P\left(x_{i}\right) S_{j}^{*}\left(y_{j}\right)=$ 0 , (iii) $\left\{x_{i} S_{j}^{*}\left(y_{j}\right) z_{k}\right\}=0$, plus

$$
\left\{x_{i} S_{i}^{*}\left(y_{i}\right) z_{k}\right\}=\left\{x_{i} y_{i} S_{k}\left(z_{k}\right)\right\} .
$$

Now in Lemma 7.7 we will see $S_{j}^{*}\left(y_{j}\right) \in M_{2}\left(\mathscr{E}_{j}\right)+M_{1}\left(\mathscr{E}_{j}\right)$, while $x_{i}, z_{k} \in J_{0}\left(\mathscr{E}_{j}\right)$, so (ii), (iii) follow automatically from Peirce orthogonality $P\left(J_{0}\right) M_{2}=P\left(J_{0}\right) M_{1}=0$, and (7.3ii-iv) reduce to (7.5). However, (7.5) is not vacuous (both sides identically zero).

Example 7.6. Let $J=\Phi e_{1} \boxplus \Phi e_{2}$ be the locally unital Jordan triple system (even unital Jordan algebra) with cover $\mathscr{E}=\left\{e_{1}, e_{2}\right\}$, and let $M=\Phi m$ be the bimodule determined by $p\left(e_{i}\right)=l\left(e_{i}, e_{j}\right)=0$, $p\left(e_{i}, e_{j}\right)=l\left(e_{i}, e_{i}\right)=I$. Here $\widetilde{J}=J \oplus M$ imbeds in $H_{2}(\Phi)$ via $e_{i} \rightarrow e_{i i}$, $m \rightarrow e_{12}+e_{21}$, and $M=M_{1}\left(e_{1}\right)=M_{1}\left(e_{2}\right)$. Then the map $S\left(\alpha e_{1}+\beta e_{2}\right)=$ $(\alpha+\beta) m$ is structural with $S^{*}=S$, and $D\left(\alpha e_{1}+\beta e_{2}\right)=(\alpha-\beta) m$ is a derivation, but $S\left(J_{1}\right)=S\left(J_{2}\right)=D\left(J_{1}\right)=D\left(J_{2}\right)=\Phi m$ so that $\left\{J_{i} J_{i} S\left(J_{i}\right)\right\}=$ $\left\{J_{i} S^{*}\left(J_{i}\right) J_{j}\right\}=\Phi m \neq 0$, and condition (7.5) is not vacuous. Note that $S=L\left(m, e_{1}+e_{2}\right)$ and $D=L\left(m, e_{1}\right)-L\left(e_{1}, m\right)$ are inner.

This example suggests that the troublesome nonorthogonality of the pieces $S_{1}, \cdots, S_{n}$ in (7.3) is due to an inner multiplication. This is in fact the case: once we remove a suitable inner multiplication, the remaining pieces $S_{i}$ map $J_{i}$ into $M_{2}\left(\mathscr{E}_{i}\right)$ and hence are automatically orthogonal. We need to investigate in more detail the interaction of a structural $S$ with a family of tripotents. First we consider a single tripotent.

Straightening Lemma 7.7. If $S \in \operatorname{Strl}(J, M)$ and $e \in J$ is tripotent, then

$$
\begin{gathered}
\text { (i) } S(e)=m_{2}+m_{1}, S^{*}(e)=m_{2}^{*}+m_{1}^{*} \text { for } m_{2}^{*}=\bar{m}_{2}=P(e) m_{2}, \\
\quad m_{i}, m_{i}^{*} \in M_{i}(e) \\
\text { (ii) } S\left(J_{2}(e)\right) \subset M_{2}(e)+M_{1}(e) \text { with } E_{1} S\left(x_{2}\right)=\left\{m_{1} e x_{2}\right\} \\
\text { (iii) } S\left(J_{0}(e)\right) \subset M_{0}(e)+M_{1}(e) \text { with } E_{1} S\left(x_{0}\right)=\left\{e m_{1}^{*} x_{0}\right\} \\
\text { (iv) } S\left(J_{1}(e)\right) \subset M_{2}(e)+M_{1}(e)+M_{0}(e) \text { with } E_{2} S\left(x_{1}\right)=\left\{e m_{1}^{*} x_{1}\right\}, E_{0} S\left(x_{1}\right)= \\
\quad\left\{m_{1} e x_{1}\right\} .
\end{gathered}
$$

If $J$ is locally unital with cover $\mathscr{E}$ then

$$
S(J) \subset M_{2}(\mathscr{E})+M_{1}(\mathscr{E}) .
$$


$S$ and $S^{*}$ preserve Peirce spaces relative to $e, S\left(J_{k}(e)\right)+S^{*}\left(J_{k}(e)\right) \subset$ $M_{k}(e)$ for $k=2,1,0$, iff $S$ and $S^{*}$ preserve $e$ in the sense that $S(e)$, $S^{*}(e) \in M_{2}(e) . \quad$ Always $S^{\prime}=S-\left\{L\left(m_{1}, e\right)+L\left(e, m_{1}\right)\right\}$ has $S^{\prime}(e), S^{*}(e) €$ $M_{2}(e)$ so we can modify $S$ by an inner multiplication to obtain $S^{\prime}$ preserving Peirce spaces. If $S=D$ is a derivation then $L\left(m_{1}, e\right)+$ $L\left(e, m_{1}\right)=D\left(m_{1}, e\right)$ is a standard inner derivation.

Proof. If $S(e)=m=\sum m_{i}, S^{*}(e)=m^{*}=\sum m_{i}^{*}$ for $m_{i}, m_{i}^{*} \epsilon$ $M_{i}(e)$ then by (7.1) $S(P(e) e)+P(e) S^{*}(e)=\{S(e) e e\}$ implies $\left(m_{2}+m_{1}+\right.$ $\left.m_{0}\right)+\overline{m_{2}^{*}}=2 m_{2}+m_{2}$, hence $m_{0}=0$ and $\overline{m_{2}^{*}}=m_{2}$. Dually $m_{0}^{*}=0$ and $\overline{m_{2}}=m_{2}^{*}$. This establishes (i).

For $x_{i} \in J_{i}(e)$ the linearized varsion of (7.1) yields $S\left(\left\{e e x_{i}\right\}\right)+$ $\left\{e S^{*}(e) x_{i}\right\}=\left\{x_{i} e S(e)\right\}+\left\{e e S\left(x_{i}\right)\right\}$, hence $i S\left(x_{i}\right)+\left\{e m_{2}^{*} x_{i}\right\}+\left\{e m_{1}^{*} x_{i}\right\}=$ $\left\{m_{2} e x_{i}\right\}+\left\{m_{1} e x_{i}\right\}+L(e, e) S\left(x_{i}\right)$. But $L\left(e, m_{2}^{*}\right)=L\left(\overline{m_{2}^{*}}, e\right)=L\left(m_{2}, e\right)$ by (i) and (1.4) so

$$
\{L(e, e)-i I\} S\left(x_{i}\right)=\left\{e m_{1}^{*} x_{i}\right\}-\left\{m_{1} e x_{i}\right\} .
$$

For $i=1$ taking components in $M_{2}, M_{0}$ yields (iv). For $i=0$ we get the expression (iii) for $E_{1} S\left(x_{0}\right)$, where $S\left(x_{0}\right) \in M_{0}+M_{1}$ since by (7.1) $P(e) S\left(x_{0}\right)=-S^{*}\left(P(e) x_{0}\right)+\left\{S^{*}(e) x_{0} e\right\}=0$. For $i=2$ we get the expression (ii) for $E_{1} S\left(x_{2}\right)$, where $S\left(x_{2}\right) \in M_{2}+M_{1}$ since by (7.1) $S\left(x_{2}\right)=$ $S\left(P(e) \bar{x}_{2}\right)=-P(e) S^{*}\left(\bar{x}_{2}\right)+\left\{S(e) \bar{x}_{2} e\right\} \in P(e) M+\{e J M\}$.

If $J$ is locally unital then $S(J)=S\left(\sum_{\mathscr{E}} J_{2}\left(e_{i}\right)\right) \subset \sum_{\mathscr{8}} M_{2}\left(e_{i}\right)+M_{1}\left(e_{i}\right)=$ $M_{2}(\mathscr{E})+M_{1}(\mathscr{E})$ by (ii).

The expressions (ii)-(iv) show that once $m_{1}=m_{1}^{*}=0$, i.e., $S(e)$, $S^{*}(e)$ lie in $M_{2}(e)$, we will have $S\left(J_{k}(e)\right) \subset M_{k}(e)$ and dually for $S^{*}$.

If $m_{1}, m_{1}^{*}$ are not zero we must modify $S$. $S^{\prime}$ has $S^{\prime}(e)=S(e)-$ $\left\{L\left(m_{1}, e\right)+L\left(e, m_{1}^{*}\right)\right\} e=\left(m_{2}+m_{1}\right)-\left(m_{1}+0\right)=m_{2}, S^{*}(e)=S^{*}(e)-$ $\left\{L\left(e, m_{1}\right)+L\left(m_{1}^{*}, e\right)\right\} e=\left(m_{2}^{*}+m_{1}^{*}\right)-\left(0+m_{1}^{*}\right)=m_{2}^{*}$ lying in $M_{2}$, so $S^{\prime}$ preserves Peirce spaces. If $S=D$ was a derivation to begin with, $S^{*}=-S$, then $m_{1}^{*}=-m_{1}$, so $L\left(m_{1}, e\right)+L\left(e, m_{1}^{*}\right)=L\left(m_{1}, e\right)-$ $L\left(e, m_{1}\right)=D\left(m_{1}, e\right)$ is a standard inner derivation.

If we have a compatible family of tripotents, we can repeatedly straighten out $S$ to respect the whole family.

Compatible Straightening Lemma 7.8. If $S \in \operatorname{Strl}(J, M)$ is a structural transformation (resp. derivation) and $\mathscr{E}=\left\{e_{1}, \cdots, e_{n}\right\}$ a compatible family of tripotents in $J$, then there exists an inner multiplication (resp. standard inner derivation) $S_{0}$ such that $S^{\prime}=$ $S-S_{0}$ has $S^{\prime}\left(e_{i}\right), S^{\prime *}\left(e_{i}\right) \in M_{2}\left(e_{i}\right)$ for all $i=1, \cdots, n$, and hence respects Peirce spaces relative to the family: $S^{\prime}\left(J_{k}\left(e_{i}\right)\right)+S^{\prime *}\left(J_{k}\left(e_{i}\right)\right) \subset$ $M_{k}\left(e_{i}\right)$ for $i=1, \cdots, n$ and $k=2,1,0$, so $S^{\prime}$ and $S^{\prime *} \operatorname{map} J_{\left(i_{1}, \cdots, i_{n}\right)}$ 
into $M_{\left(i_{1}, \cdots, i_{n}\right)}$.

Proof. For a single tripotent $n=1$ this is just (7.7.) Assume the result for $n-1$ tripotents, so we can modify $S$ by something inner and assume from the start that $S\left(e_{i}\right), S^{*}\left(e_{i}\right)$ lie in $M_{2}\left(e_{i}\right)$ for $i=1,2, \cdots, n-1$. We must modify $S$ to obtain $S^{\prime}\left(e_{n}\right), S^{\prime *}\left(e_{n}\right) \epsilon$ $M_{2}\left(e_{n}\right)$ without unduly disturbing the previous action on $e_{1}, \cdots, e_{n-1}$. So consider $S^{\prime}=S-S_{0}$ for $S_{0}=L\left(m_{1}, e\right)+L\left(e, m_{1}^{*}\right)$ as in (7.7) for $S(e)=m_{2}+m_{1}, S^{*}(e)=m_{2}^{*}+m_{1}^{*}$ where we set $e=e_{n}$. We know $S_{0}$ is inner (resp. standard inner derivation) and $S^{\prime}(e), S^{\prime *}(e) \in M_{2}(e)$. We must show that we haven't disturbed the previous actions,

$$
S_{0}\left(e_{i}\right)=\left\{m_{1} e e_{i}\right\}+\left\{e m_{1}^{*} e_{i}\right\} \in M_{2}\left(e_{i}\right) \quad(i=1, \cdots, n-1)
$$

and dually for $S_{0}^{*}$.

By the induction hypothesis, $S$ preserves Peirce spaces relative to $e_{i}$. By compatibility, the same is true of $L(e, e)$ and $P(e)^{2}$. Now (7.1) yields the general identities

$$
\begin{aligned}
& {[S, L(x, y)]=L(S x, y)-L\left(x, S^{*} y\right)} \\
& {[S, P(x) P(y)]=P(S x, x) P(y)-P(x) P\left(S^{*} y, y\right)}
\end{aligned}
$$

for structural $S$, so for $x=y=e$ we see

$$
\begin{aligned}
& L(m, e)-L\left(e, m^{*}\right)=L\left(m_{1}, e\right)-L\left(e, m_{1}^{*}\right) \\
& P(m, e) P(e)-P(e) P\left(m^{*}, e\right)=P\left(m_{1}, e\right) P(e)-P(e) P\left(m_{1}^{*}, e\right)
\end{aligned}
$$

preserve Peirce spaces (using $m_{2}^{*}=\bar{m}_{2}$ by $(7.7 \mathrm{i})$ and (1.4) to get rid of $\left.m_{2}\right)$. But then $P\left(m_{1}, e\right) P(e)-P(e) P\left(m_{1}^{*}, e\right)-\left\{L\left(m_{1}, e\right)-L\left(e, m_{1}^{*}\right)\right\} L(e, e)$ also preserves Peirce spaces, and by $(0.6)$ this equals $\left\{L(e, e) L\left(m_{1}, e\right)-\right.$ $\left.L\left(e, P(e) m_{1}\right)\right\}-\left\{L\left(e, m_{1}^{*}\right) L(e, e)-L\left(P(e) m_{1}^{*}, e\right)\right\}-L\left(m_{1}, e\right) L(e, e)+L(e$, $\left.m_{1}^{*}\right) L(e, e)=L(e, e) L\left(m_{1}, e\right)-L\left(m_{1}, e\right) L(e, e)=L\left(\left\{e e m_{1}\right\}, e\right)-L\left(m_{1},\{e e e\}\right)$ (by $(0.5))=-L\left(m_{1}, e\right)$. Once this preserves Peirce spaces, so does $L\left(e, m_{1}^{*}\right)$ by $\left(^{* *}\right)$ :

$\left({ }^{* * *}\right) \quad L\left(m_{1}, e\right), L\left(e, m_{1}^{*}\right)$ preserve Peirce spaces $J_{k}\left(e_{i}\right)$.

Thus $e_{i} \in J_{2}\left(e_{i}\right)$ implies $\left\{m_{1} e e_{i}\right\},\left\{e m_{1}^{*} e_{i}\right\} \in M_{2}\left(e_{i}\right)$, yielding $\left(^{*}\right)$. Therefore the modification $S^{\prime}=S-S_{0}$ preserves Peirce spaces relative to $e_{1}, \cdots, e_{n-1}$ and $e=e_{n}$ as well. This completes the induction.

We say a structural $S: J \rightarrow M$ is locally unital with respect to a given cover $\mathscr{E}=\left\{e_{1}, \cdots, e_{n}\right\}$ of $J$ if it preserves the Peirce spaces relative to $\mathscr{E}$ :

$$
S\left(J_{\left(i_{1}, \cdots, i_{n}\right)}\right)+S^{*}\left(J_{\left(i_{1}, \cdots, i_{n}\right)}\right) \subset M_{\left(i_{1}, \cdots, i_{n}\right)} .
$$


By (7.8) this is equivalent to $S$ preserving the individual Peirce spaces

$$
S\left(J_{2}\left(e_{i}\right)\right)+S^{*}\left(J_{2}\left(e_{i}\right)\right) \subset M_{2}\left(e_{i}\right)
$$

or even just to preserving the tripotents,

$$
S\left(e_{i}\right), S^{*}\left(e_{i}\right) \in M_{2}\left(e_{i}\right) \text {. }
$$

Notice that if $J$ is unital $(\mathscr{E}=\{e\})$ then $J=J_{2}(e)$ and unitality just means $S(J) \subset M_{2}(e)$ or $S(e) \in M_{2}(e)$ (and dually for $S^{*}$ ):

Proposition 7.10. A linear transformation $S$ from a direct sum $J=J_{1} \boxplus \cdots \boxplus J_{n}$ of locally unital Jordan triple systems into a bimodule $M$ is structural (resp. a derivation) iff it has the form $S=S_{0} \oplus S_{1} \oplus \cdots \oplus S_{n}$ where $S_{0}$ is inner (resp. standard inner derivation) and $S_{i}: J_{i} \rightarrow M$ are locally unital and structural (resp. derivations). In particular, any $S$ is the sum of an inner $S_{0}$ and a locally unital $S^{\prime}$.

Proof. Sufficiency is easy: $S$ is structural or a derivation iff $S^{\prime}=S-S_{0}$ is, and the condition (7.5) on $S^{\prime}=S_{1} \oplus \cdots \oplus S_{n}$ is automatic (both sides vanish by orthogonality because $S_{i}^{*}\left(y_{i}\right) \in M_{2}\left(\mathscr{E}_{i}\right)$, $S_{k}\left(z_{k}\right) \in M_{2}\left(\mathscr{E}_{k}\right)$ by local unitality).

For necessity we apply the Straightening Proposition 7.8 to the compatible family $\mathscr{E}=\mathscr{E}_{1} \cup \cdots \cup \mathscr{E}_{n}$ (recalling (2.3)).

We can use these results straightening structural transformations to prove additivity of the cohomology groups $H^{1}$, just as we used liftings of tripotents to straighten linear lifts and thereby prove additivity of $H^{2}$. Recall that there are two slightly different first cohomology groups

$$
\begin{aligned}
& H^{1}(J, M)=\operatorname{Der}(J, M) / \operatorname{Inder}(J, M) \\
& \hat{H}^{1}(J, M)=\operatorname{Strl}(J, M) / \operatorname{Instrl}(J, M) .
\end{aligned}
$$

7.11. Direct Decomposition Theorem for $H^{1}$. If $J_{1}, \cdots, J_{n}$ are locally unital Jordan triple systems relative to covers $\mathscr{E}_{1}, \cdots \mathscr{E}_{n}$ and $M$ is a bimodule for the direct sum $J=J_{1} \boxplus \cdots \boxplus J_{n}$, then

$$
\begin{aligned}
& H^{1}(J, M) \cong H^{1}\left(J_{1}, M_{1}\right) \oplus \cdots \oplus H^{1}\left(J_{n}, M_{n}\right) \\
& \hat{H}^{1}(J, M) \cong \hat{H}^{1}\left(J_{1}, M_{1}\right) \oplus \cdots \oplus \hat{H}^{1}\left(J_{n}, M_{n}\right)
\end{aligned}
$$

where $M_{i}=M_{i i}+M_{i 0}+M_{00}=\bigcap_{j \neq i} M_{0}\left(\mathscr{E}_{j}\right)$.

Proof. As in (6.2) we begin by imbedding $\oplus \hat{H}^{1}\left(J_{i}, M_{i}\right)$ in 
$\hat{H}^{1}(J, M)$. We have a natural imbedding $\oplus S_{i} \rightarrow S$ of $\oplus \operatorname{Strl}\left(J_{i}, M_{i}\right)$ in $\operatorname{Strl}(J, M)$ (the orthogonality condition (7.5) being automatically satisfied thanks to $S_{j}(J), S_{j}^{*}(J) \subset M_{j}=\bigcap_{l \neq j} M_{0}\left(\mathscr{E}_{l}\right)$ and $J_{j}=J_{2}\left(\mathscr{E}_{j}\right)$ by hypothesis on $\left.S_{j}\right)$. This induces a linear map $\oplus \operatorname{Strl}\left(J_{i}, M_{i}\right) \rightarrow$ $\hat{H}^{1}(J, M)$.

To characterize the kernel of this map, we need to know when $S=\bigoplus S_{i}$ is inner on $J=\boxplus J_{i}$, i.e., a sum of $L\left(x_{k k}, m_{k l}\right), L\left(n_{l k}, y_{k k}\right)$ for various elements $x_{k l k}, y_{k l} \subset J_{k}=J_{k k}, m_{k l}, n_{l k} \in M_{k l}$ the Peirce spaces (2.5) relative to the given orthogonal covers $\mathscr{E}_{1}, \cdots, \mathscr{E}_{n}$ for $J$. (Remember by (2.6) $L\left(a_{k l}, b_{i j}\right)=0$ unless the indices are linked, $\{k, l\} \cap\{i, j\} \neq \varnothing$, so we need only consider $m_{i j}$ with at least one index $k$ ). We wish to show innerness on $J$ implies innerness on each $J_{i}$. Restricting the identity $S=\sum L\left(x_{k k}, m_{k l}\right)+L\left(n_{l k}, y_{k k}\right)$ expressing innerness of $S$ on $J$ to elements $z_{i i}$ in the ideal $J_{i}$, and then applying the Peirce projection operator $E_{i i}+E_{i 0}+E_{00}$ of $M$ on $M_{i}$, yields

$$
\text { (*) } S_{i}\left(z_{i i}\right)=\sum\left\{L\left(x_{i i}, m_{i i}\right)+L\left(x_{i i}, m_{i 0}\right)+L\left(n_{i i}, y_{i i}\right)+L\left(n_{0 i}, y_{i i}\right)\right\} z_{i i}
$$

since by (2.6) $\left\{x_{k k} m_{k l} z_{i\}}\right\}$ vanishes when $k=i$ unless $l=i$ or 0 , whence $\left\{x_{i i} m_{i l} z_{i i}\right\}$ falls in $J_{i i}+J_{i 0}$ by (2.8) (P1), (U1), and vanishes when $k \neq i$ unless $l=i$, whence $\left\{x_{k k} m_{k i} z_{i i}\right\} \in M_{k i}$ by (2.8) (P4), so only $k=i, l=i, 0$ effectively produce elements of $M_{i i}+M_{i 0}+M_{00}$, while $\left\{n_{l l} y_{k k} z_{i i}\right\}$ vanishes unless $k=i$ by (2.6) and then falls in $M_{l i}$ if $l \neq i, 0$ by (2.8) (P5) or $M_{i i}+M_{i 0}$ if $l=i, 0$ by (2.8)(P5), (P1), so only $k=i, l=i, 0$ are effective. But such $m_{i i}, m_{i 0}, n_{i i}, n_{0 i}$ all lie in $M_{i}$, so $\left.{ }^{*}\right)$ shows $S_{i} \in \operatorname{Instrl}\left(J_{i}, M_{i}\right)$ is inner on $J_{i}$.

Conversely, if each $S_{i}$ is inner as in $\left(^{*}\right)$ then their sum $S$ is inner on $J$ (note the $L\left(x_{j j}, m_{j j}\right), L\left(x_{j j}, m_{j 0}\right), L\left(n_{j j}, y_{j j}\right), L\left(n_{0 j}, y_{j j}\right)$ annihilate $J_{i i}$ for $j \neq i$ by (2.6) and hence do not contribute to the action of $S$ on $\left.J_{i}\right)$.

Thus $\oplus \operatorname{Strl}\left(J_{i}, M_{i}\right) \rightarrow \hat{H}^{1}(J, M)$ has kernel precisely $\oplus \operatorname{Instrl}\left(J_{i}\right.$, $\left.M_{i}\right)$ and thus induces an imbedding of $\oplus \hat{H}^{1}\left(J_{i}, M_{i}\right) \cong\left\{\oplus \operatorname{Strl}\left(J_{i}, M_{i}\right)\right\} /$ $\left\{\oplus \operatorname{Instrl}\left(J_{i}, M_{i}\right)\right\}$ in $\hat{H}^{1}(J, M)$.

By (7.10) this imbedding is surjective: if $S \in \operatorname{Strl}(J, M)$ then $S$ is congruent modulo $\operatorname{Instrl}(J, M)$ to a sum $S_{1} \oplus \cdots \oplus S_{n}$ of locally unital $S_{i}$, where by local unitality of $S_{i}$ with respect to $\mathscr{E}_{i}$ we have $S_{i}, S_{i}^{*}$ mapping $J_{i}=J_{2}\left(\mathscr{E}_{i}\right)$ into $M_{2}\left(\mathscr{E}_{i}\right)=M_{i i} \subset M_{i}$, so $S_{i} \in$ $\operatorname{Strl}\left(J_{i}, M_{i}\right)$. Thus we have a natural isomorphism $\oplus \hat{H}^{1}\left(J_{i}, M_{i}\right) \cong$ $\hat{H}^{1}(J, M)$.

The same argument, mutatis mutandis, shows $\oplus H^{1}\left(J_{i}, M_{i}\right) \cong$ $H^{1}(J, M)$.

Just as in (6.3), we can apply this to semisimple Jordan pairs 
and triple systems.

7.12. Direct Decomposition Theorem For $H^{1}$. If $J=J_{1} \boxplus \cdots \boxplus J_{n}$ is the decomposition into simple ideals of a semisimple Jordan pair with d.c.c. (or a semisimple Jordan triple system finite-dimensional over an algebraically closed field of characteristic $\neq 2$ ), then for any $J$-bimodule $M$ we have

$$
H^{1}(J, M) \cong \bigoplus H^{1}\left(J_{i}, M_{i}\right), \hat{H}^{1}(J, M) \cong \bigoplus \hat{H}^{1}\left(J_{i}, M_{i}\right)
$$

for $M_{i}=M_{i i}+M_{i 0}+M_{00}=\bigcap_{j \neq i} M_{0}\left(\mathscr{E}_{j}\right)$ for compatible covers $\mathscr{E}_{j}$ of $J_{j}$.

Our results have reduced the study of the cohomology $H^{1}, \hat{H}^{1}$, $H^{2}$ of semisimple $J$ to that of the simple $J_{i}$. We will investigate the cohomology of simple systems in a separate paper [5].

It should be noted that Kühn and Rosendahl [1] have proved directly that $H^{1}(J, M)=H^{2}(J, M)=0$ for all finite-dimensional semisimple Jordan pairs and triple systems $J$ of characteristic 0 , using the trivial cohomology of the Tits-Koecher Lie algebra $K(J)$ to deduce triviality of the cohomology of $J$.

\section{REFERENCES}

1. O. Kühn and A. Rosendahl, Wedderburnzerlegung für Jordan-Paare, Manuscripta Mathematica, 24 (1978), 403-435.

2. O. Loos, Jordan Pairs, Springer Lecture Notes in Mathematics, No. 460, Springer Verlag, New York, 1975.

3. - Lectures on Jordan Triple Systems, University of British Columbia Lecture Notes, Vancouver, 1971.

4. K. McCrimmon, Peirce ideals in Jordan triple systems, Pacific J. Math., 83 (1979), 415-439.

5. Wedderburn non-splittings and cohomology of Jordan algebras, to appear.

6. Representations of quadratic Jordan algebras, Trans. Amer. Math. Soc., 153 (1971), 279-305.

7. K. McCrimmon and $\mathrm{K}$. Meyberg, Coordinatization of Jordan triple systems, Comm. in Algebra, 9 (14) (1981), 1495-1542.

8. K. Meyberg, Lectures on algebras and triple systems, University of Virginia Lecture Notes, Charlottesville, 1972.

Received March 7, 1980 and in revised form June 18, 1981. This research was partially supported by grants from the National Science Foundation and the Alexander von Hum. boldt Stiftung.

UNIVERSITY OF VIRGINIA

Charlottesville, VA 22903 


\section{PACIFIC JOURNAL OF MATHEMATICS}

\section{EDITORS}

DONALD BABBITT (Managing Editor)

University of California

Los Angeles, California 90024

Hugo RossI

University of Utah

Salt Lake City, UT 84112

C. C. Moore and Arthur Agus

University of California

Berkeley, CA 94720
J. DugundJI

Department of Mathematics University of Southern California Los Angeles, California 90007

R. FInN and J. Milgram Stanford University Stanford, California 94305

ASSOCIATE EDITORS
R. ARNES
E. F. BeCKenbach
B. H. NeumanN
F. WOLF
K. YoSHIDA

\section{SUPPORTING INSTITUTIONS}

UNIVERSITY OF ARIZONA

UNIVERSITY OF BRITISH COLUMBIA

CALIFORNIA INSTITUTE OF TECHNOLOGY

UNIVERSITY OF CALIFORNIA

MONTANA STATE UNIVERSITY

UNIVERSITY OF NEVADA, RENO

NEW MEXICO STATE UNIVERSITY

OREGON STATE UNIVERSITY
UNIVERSITY OF OREGON

UNIVERSITY OF SOUTHERN CALIFORNIA

STANFORD UNIVERSITY

UNIVERSITY OF HAWAII

UNIVERSITY OF TOKYO

UNIVERSITY OF UTAH

WASHINGTON STATE UNIVERSITY

UNIVERSITY OF WASHINGTON 


\section{Pacific Journal of Mathematics}

\section{Vol. 103, No. $1 \quad$ March, 1982}

Abdul Aziz, On the zeros of composite polynomials ..................

Salomon Benzaquen and Enrique M. Cabaña, The expected measure of the level sets of a regular stationary Gaussian process $\ldots \ldots \ldots \ldots \ldots$

Claudio D’Antoni, Roberto Longo and László Zsidó, A spectral mapping theorem for locally compact groups of operators $\ldots \ldots \ldots \ldots \ldots \ldots \ldots 17$

Ronald Dotzel, Semifree finite group actions on homotopy spheres ........ 25

Daniel H. Gottlieb, The Lefschetz number and Borsuk-Ulam theorems . . . . . 29

Shui-Hung Hou, On property $(Q)$ and other semicontinuity properties of

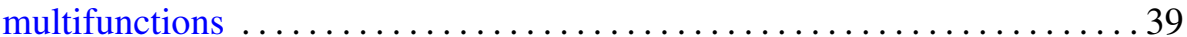

Kevin Mor McCrimmon, Compatible Peirce decompositions of Jordan triple systems

Mitsuru Nakai, Corona problem for Riemann surfaces of Parreau-Widom

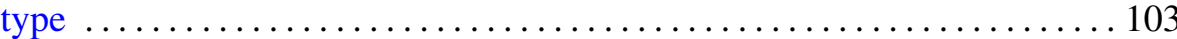

Jack Ray Porter and R. Grant Woods, Extensions of Hausdorff spaces . . . . 111

Milton Rosenberg, Quasi-isometric dilations of operator-valued measures

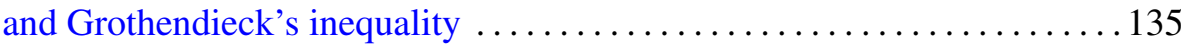

Joseph L. Taylor, A bigger Brauer group $\ldots \ldots \ldots \ldots \ldots \ldots \ldots \ldots \ldots \ldots$

Thomas Vogel, Symmetric unbounded liquid bridges . . . . . . . . . . . 205

Steve Wright, The splitting of operator algebras. II ............... 243 